\title{
The CMS Tracker operation and performance at the Magnet Test and Cosmic Challenge
}

W. Adam, T. Bergauer, M. Dragicevic, M. Friedl, R. Frühwirth, S. Hänsel, J. Hrubec, M. Krammer, M. Pernicka, W. Waltenberger and E. WidI Institut für Hochenergiephysik der Österreichischen Akademie der Wissenschaften (HEPHY), Vienna, Austria

P. Van Mechelen, M. Cardaci, W. Beaumont, E. de Langhe, E.A. de Wolf and E. Delmeire Universiteit Antwerpen, Belgium

O. Bouhali, O. Charaf, B. Clerbaux, J.-P. Dewulf, S. Elgammal, G. Hammad, G. de Lentdecker, P. Marage, C. Vander Velde, P. Vanlaer and J. Wickens Université Libre de Bruxelles, Bruxelles, Belgium

O. Devroede, S. De Weirdt, J. D’Hondt, R. Goorens, J. Heyninck, J. Maes, S. Tavernier, L. Van Lancker, P. Van Mulders and I. Villella Vrije Universiteit Brussel, Brussel, Belgium

S. Assouak, J.-L. Bonnet, G. Bruno, B. De Callatay, S. De Visscher, D. Favart, B. Florins, E. Forton, A. Giammanco, G. Gregoire, S. Kalinin, D. Kcira, Th. Keutgen, V. Lemaitre, D. Michotte, O. Militaru, S. Ovyn, K. Piotrzkowski, X. Rouby,

D. Teyssier ${ }^{d}$ and O. Van der Aa Université Catholique de Louvain, Louvain-la-Neuve, Belgium

E. Daubie

Université de Mons-Hainaut, Mons, Belgium

E. Anttila, S. Czellar, J. Härkönen, V. Karimäki, T. Lampén, T. Lindén, P. -R. Luukka, T. Mäenpää, E. Tuominen and J. Tuominiemi University of Helsinki-HIP, Finnland

G. Boudoul, E. Chabanat, E. Chabert, R. Chierici, D. Contardo ${ }^{a}$, N. Estre,

R. Haroutunian, N. Lumb, S. Perries and B. Trocme

Institut de Physique Nucléaire, Université Claude Bernard Lyon 1, IN2P3-CNRS, Villeurbanne, France 


\section{J.-L. Agram, R. Blaes, F. Drouhin ${ }^{a}$ and J.-C. Fontaine}

Groupe de Recherches en Physique des Hautes Energies, Université de Haute Alsace,

Mulhouse, France

J.-D. Berst, J.-M. Brom, U. Goerlach, P. Graehling, L. Gross, J. Hosselet, P. Juillot, C. Maazouzi, C. Ollivetto and P. Van Hove

Institut Pluridisciplinaire Hubert Curien, Université Louis Pasteur Strasbourg,

IN2P3-CNRS, France

G. Anagnostou, R. Brauer, H. Esser, L. Feld, W. Karpinski, K. Klein, C. Kukulies,

J. Olzem, A. Ostapchuk, D. Pandoulas, G. Pierschel, F. Raupach, S. Schael,

G. Schwering, M. Thomas, M. Weber, B. Wittmer and M. Wlochal

I. Physikalisches Institut, RWTH Aachen University, Germany

F. Beissel, E. Bock, G. Flugge, T. Hermanns, D. Heydhausen, D. Jahn, G. Kaussen,

A. Linn, L. Perchalla, M. Poettgens, O. Pooth, A. Stahl and M.H. Zoeller

III. Physikalisches Institut, RWTH Aachen University, Germany

E. Butz, S. Fricke, G. Flucke, R. Klanner, U. Pein, P. Schleper and G. Steinbrück University of Hamburg, Institute for Experimental Physics, Hamburg, Germany

P. Blüm, W. De Boer, A. Dierlamm, G. Dirkes, M. Fahrer, M. Frey, A. Furgeri,

F. Hartmann ${ }^{a}$, S. Heier, K.-H. Hoffmann, B. Ledermann, T. Liamsuwan, S. Müller,

T. Müller, A.U. Scheurer, F.-P. Schilling, H.-J. Simonis and V. Zhukov

Karlsruhe-IEKP, Germany

G. De Robertis, L. Fiore, L. Silvestris, P. Tempesta and G. Zito INFN Bari, Italy

D. Creanza, N. De Filippis ${ }^{b}$, M. De Palma, D. Giordano, G. Maggi, N. Manna, S. My, V. Radicci ${ }^{i}$ and G. Selvaggi INFN and Dipartimento Interateneo di Fisica, Bari, Italy

S. Albergo, M. Chiorboli, S. Costa, M. Galanti, F. Noto, R. Potenza, M.A. Saizu, ${ }^{h}$

C. Sutera, A. Tricomi and C. Tuvé

INFN and University of Catania, Italy

C. Civinini, F. Manolescu, M. Meschini, S. Paoletti and G. Sguazzoni INFN Firenze, Italy

B. Broccolo, V. Ciulli, 'R. D’Alessandro, E. Focardi, S. Frosali, C. Genta, G. Landi,

P. Lenzi, A. Macchiolo, N. Magini, S. Mersi and G. Parrini

INFN and University of Firenze, Italy

P. Azzi, N. Bacchetta, ${ }^{a}$ A. Candelori, T. Dorigo and A. Kaminsky

INFN Padova, Italy 
D. Bisello, M. De Mattia, P. Giubilato, V. Khomenkov, M. Loreti, S. Mattiazzo, M. Nigro, A. Paccagnella and M. Tosi

INFN and University of Padova, Italy

G.M. Bilei, ${ }^{a}$ B. Checcucci, L. Fanò and L. Servoli

INFN Perugia, Italy

F. Ambroglini, E. Babucci, D. Benedetti, M. Biasini, B. Caponeri, R. Covarelli, M. Giorgi, P. Lariccia, G. Mantovani, M. Marcantonini, V. Postolache, A. Santocchia and D. Spiga

INFN and University of Perugia, Italy

G. Bagliesi, ${ }^{a}$ T. Boccali, F. Bosi, R. Castaldi, C. Cerri, R. Dell'Orso, D. Dobur,

S. Dutta, A. Giassi, A.C. Kraan, T. Lomtadze, G. Magazzu, G. Martinelli, A. Moggi,

F. Palla, F. Palmonari, F. Raffaelli, G. Sanguinetti, S. Sarkar, A.T. Serban, ${ }^{j}$

P. Spagnolo, R. Tenchini, A. Venturi, P.G. Verdini ${ }^{a}$ and M. Vos INFN Pisa, Italy

A. Basti, L. Benucci, J. Bernardini, L. Borrello, F. Fiori, S. Linari, M. Massa,

A. Messineo, G. Segneri and G. Tonelli ${ }^{a}$

University of Pisa and INFN Pisa, Italy

P. Azzurri, A. Bocci, L. Foa, S. Gennai, F. Ligabue, B. Mangano, ${ }^{e}$ G. Petrucciani and

A. Rizzi $^{k}$

Scuola Normale Superiore di Pisa and INFN Pisa, Italy

N. Demaria

INFN Torino, Italy

M.A. Borgia, R. Castello, M. Costa, E. Migliore and A. Romero

INFN and University of Torino, Italy

D. Abbaneo, I. Ahmed, C. Bloch, H. Breuker, O. Buchmuller, A. Cattai, C. Delaere, L.M. Edera, M. Eppard, K. Gill, A.-S. Giolo-Nicollerat, R. Grabit, A. Honma, M. Huhtinen, K. Kloukinas, C. Ljuslin, M. Mannelli, L. Masetti, A. Marchioro,

S. Michal, L. Mirabito, A. Onnela, C. Paillard, I. Pal, P. Petagna, M. Pioppi,

H. Postema, R. Ranieri, D. Ricci, G. Rolandi, F. Ronga, ${ }^{f}$ P. Siegrist, P. Tropea,

J. Troska, A. Tsirou, M. Vander Donckt and F. Vasey

European Organization for Nuclear Research (CERN), Geneva, Switzerland

I. Church, J. Cole, J. Coughlan, A. Gay, S. Taghavi and I. Tomalin

STFC, Rutherford Appleton Laboratory, Chilton, Didcot, United Kingdom

R. Bainbridge, N. Cripps, J. Fulcher, G. Hall, M. Noy, M. Pesaresi, V. Radicci,

D.M. Raymond, A. Rose, P. Sharp, ${ }^{a}$ M. Stoye, M. Wingham and O. Zorba

Imperial College, London, United Kingdom 
I. Goitom, P.R. Hobson, I. Reid and L. Teodorescu

Brunel University, Uxbridge, United Kingdom

M. Bose, G. Hanson, G.-Y. Jeng, H. Liu, G. Pasztor, A. Satpathy and R. Stringer University of California, Riverside, California, U.S.A.

C. Campagnari, M. D’Alfonso, J. Garberson, J. Incandela, P. Kalavase, D. Kovalskyi, S. Kyre, J. Lamb, S. Lowette, F. Rebassoo, J. Ribnik, J. Richman, R. Rossin,

D. Stuart, S. Swain, J.-R. Vlimant and D. White

University of California, Santa Barbara, California, U.S.A.

\section{S.R. Wagner}

University of Colorado, Boulder, Colorado, U.S.A.

L. Bagby, P. Bhat, K. Burkett, O. Gutsche, H. Jensen, M. Johnson, D. Mason,

T. Miao, C. Noeding, E. Skup, W. Spalding, L. Spiegel, S. Tkaczyk and F. Yumiceva Fermi National Accelerator Laboratory (FNAL), Batavia, Illinois, U.S.A.

I. Anghel, V.E. Bazterra, C.E. Gerber, S. Khalatian and E. Shabalina University of Illinois, Chicago, Illinois, U.S.A.

P. Baringer, A. Bean, J. Chen and T. Moulik University of Kansas, Lawrence, Kansas, U.S.A.

T. Bolton, D. Onoprienko and Z. Wan Kansas State University, Manhattan, Kansas, U.S.A.

A. Gritsan,C.K. Lae and N. Tran

Johns Hopkins University, Baltimore, Maryland, U.S.A.

P. Everaerts, K.A. Hahn, P. Harris, S. Nahn, M. Rudolph, C. Roland and K. Sung Massachusetts Institute of Technology, Cambridge, Massachusetts, U.S.A.

B. Betchart, R. Demina, Y. Gotra, S. Korjenevski, D. Miner and D. Orbaker University of Rochester, New York, U.S.A.

L. Christofek, R. Hooper, G. Landsberg, D. Nguyen, M. Narain, T. Speer and K.V. Tsang

Brown University, Providence, Rhode Island, U.S.A.

${ }^{a}$ Also at CERN, European Organization for Nuclear Research, Geneva, Switzerland

${ }^{b}$ Now at LLR-Ecole Polytechnique, France

${ }^{c}$ Also at Research Institute for Particle and Nuclear Physics, Budapest, Hungary

${ }^{d}$ Now at II. Physikalisches Institut, RWTH Aachen University, Germany

${ }^{e}$ Now at University of California, San Diego, U.S.A.

${ }^{f}$ Now at Eidgenössische Technische Hochschule, Zürich, Switzerland 
${ }^{g}$ Also at Paul Scherrer Institut, Villigen, Switzerland

${ }^{h}$ On leave from IFIN-HH, Bucharest, Romania

${ }^{i}$ Now at University of Kansas, U.S.A.

${ }^{j}$ On leave from National Institute for Nuclear Physics and Engineering-Horia Hulubei

(IFIN-HH), Magurele-Bucharest, Romania

${ }^{k}$ Now at ETH Zurich, Zurich, Switzerland

E-mail: vitaliano.ciulliefi.infn.it

ABSTRACT: During summer 2006 a fraction of the CMS silicon strip tracker was operated in a comprehensive slice test called the Magnet Test and Cosmic Challenge (MTCC). At the MTCC, cosmic rays detected in the muon chambers were used to trigger the readout of all CMS subdetectors in the general data acquisition system and in the presence of the $4 \mathrm{~T}$ magnetic field produced by the CMS superconducting solenoid. This document describes the operation of the Tracker hardware and software prior, during and after data taking. The performance of the detector as resulting from the MTCC data analysis is also presented.

KEYWORDS: Particle tracking detectors; Solid state detectors.

${ }^{*}$ Corresponding author 


\section{Contents}

1. Introduction 2

2. Tracker setup 2

2.1 Detector layout 2

2.2 Detector Control and Safety Systems

2.3 Detector Readout System 6

2.4 Trigger, Data Acquisition and Computing systems 7

3. Offline software 10

3.1 Reconstruction 10

3.1.1 Local reconstruction 11

3.1.2 Track reconstruction 12

3.1 .3 Alignment 13

3.2 Simulation 14

3.3 Data Quality Monitoring and visualization 18

3.4 Event selection 20

4. Detector commissioning 21

4.1 Configuration of readout electronics

4.2 Synchronization with external trigger 22

5. Tracker performance 24

5.1 Noise studies and problematic detectors

5.1 .1 TIB modules 26

5.1 TOB modules 28

5.1 .3 TEC modules 31

5.2 Tracking and alignment

5.2.1 TIB survey measurements 35

5.2 .2 Pre-alignment in the tracker assembly hall 37

5.2 .3 Local alignment sensitivity with assembly data

5.2.4 Alignment analysis of MTCC data

5.2.5 Track reconstruction results 39

5.2.6 Comparison of tracks reconstructed in the tracker and in the muon chambers 40

5.3 TIB and TOB performance

5.4 TEC performance 50

5.5 Gain measurement 52

5.6 Lorentz angle measurement 54

6. Conclusions 55 


\section{Introduction}

An early combined operation of all the CMS subsystems was considered to be an invaluable opportunity to anticipate unforeseen problems and get the experiment ready to take high-quality data as early as possible after the LHC start-up. With this aim in mind, starting in summer 2006 the CMS collaboration took advantage of the magnet commissioning tests and of the partial installation of some of the subdetectors in the SX5 surface hall to launch the Magnet Test and Cosmic Challenge (MTCC). At the MTCC, a fraction of all subdetectors (with the exception of the pixel systems) was operated with an up-to $4 \mathrm{~T}$ magnetic field delivered by the superconducting solenoid and read out with a reduced scale implementation of the final global data acquisition system (DAQ). Cosmic muon triggering was provided by the Level-1 trigger electronics of the muon detectors.

This document describes the operation and performance of the silicon strip tracker (hereafter referred to as the "tracker") at the MTCC.

The MTCC consisted of two different phases. In phase I the magnet was commissioned up to design-value for the electric current and B field. In phase II the magnetic field was mapped inside and outside of the solenoid. The tracker participated only in phase I of the MTCC. Data taking in this period spanned nearly the entire month of August 2006. Although the MTCC tracker setup represented only about $1 \%$ of the final system, most of the selected hardware and software systems were advanced prototypes of the final versions. Similarly, the procedures for setting up, monitoring, and controlling the tracker, as well as those pertinent to data handling, were the ones planned for the actual operation of CMS. In addition, the MTCC offered the unique opportunity of testing the performance of the tracker in the presence of the $4 \mathrm{~T}$ magnetic field. The MTCC also represented an important milestone for the new CMS offline software, CMSSW [1], the architecture of which had been totally rewritten beginning in early 2005. The new software was designed to allow all detectors to be read out in the global DAQ, to unpack raw data, and to facilitate data quality monitoring and event reconstruction using calibration and alignment data.

The MTCC tracker layout and its main subsystems are described in section 2 . The offline software, which is central for the production of results on the detector performance, is described section 3. Commissioning of the tracker, which included the procedures followed to tune the readout electronics, to synchronize the data readout to the Level-1 trigger signals and to provide the initial alignment constants, is the subject of section $\emptyset$. Tracker performance results are presented in section 5 .

\section{Tracker setup}

\subsection{Detector layout}

The CMS tracker [2, 3] has an active surface of $210 \mathrm{~m}^{2}$ of silicon strip detectors, instrumented with about $10^{7}$ read-out channels. The tracker setup for the MTCC represents $1 \%$ of the electronic channels in the full tracker. The active area of the MTCC tracker detector consists of $0.75 \mathrm{~m}^{2}$ of silicon sensors. These were arranged in three basic structural units corresponding to the major subsystems of the CMS tracker: the Tracker Inner Barrel (TIB), the Tracker Outer Barrel (TOB) and the Tracker Endcap (TEC). This layout is shown in figure 1 and summarized in table 11. Throughout this document, the standard CMS reference system is used. This system has its origin in the centre 


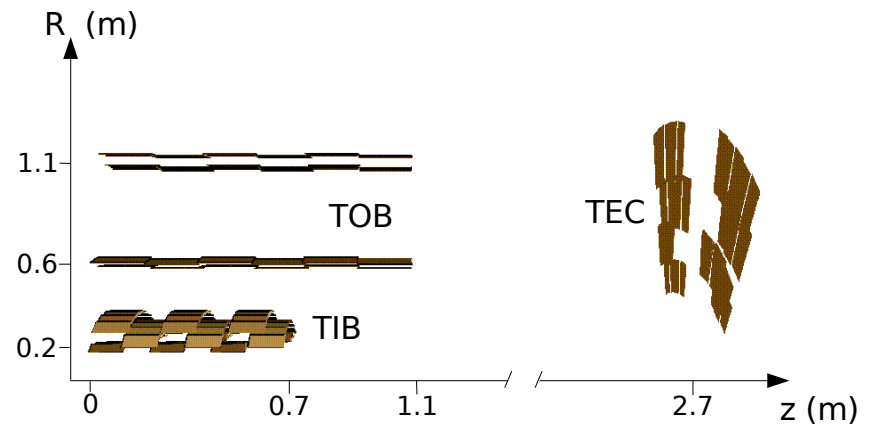

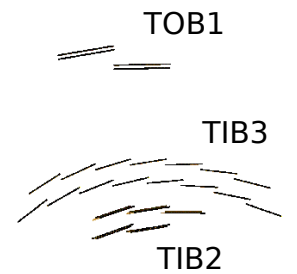

Figure 1. Layout of the Tracker MTCC setup: (a) 3D view (the $z$ axis goes from left to right); (b) $x y$ view of the barrel part. The instrumented parts are a fraction of layer 2 and layer 3 of TIB, two rods in layer 1 and in layer 5 of TOB, two petals in disk 9 of TEC.

of the detector, the $z$ axis is along the beam line in the anti-clockwise direction for an observer standing in the middle of the LHC ring. The $x$ axis points to the LHC centre and the $y$ axis points upward. The azimuthal angle $\phi$ is measured starting from the $x$ axis toward the $y$ axis. The polar radius $r$ is defined as the distance from the $z$ axis in the transverse $(x, y)$ plane.

The TIB structure consisted of two mechanical prototype shells corresponding to layers 2 (L2) and 3 (L3) of the entire TIB. These shells were partly populated with modules: layer 2 contained 15 double-sided modules and layer 3 contained 45 single-sided modules.

The TOB mechanical structure represented a 40-degree slice of the CMS TOB. It could hold up to eight TOB sub-structures ("rods") in locations corresponding to layers 1 and 5 of the TOB. During the MTCC there were two rods inserted in the first layer (L1) and two rods in the fifth layer (L5). The L1 rods each contained six single-sided modules with a strip pitch of $183 \mu \mathrm{m}$, and the L5 rods contained six single-sided modules with a strip pitch of $122 \mu \mathrm{m}$.

The TEC structure consisted of three custom-made disks corresponding to the outer three disks $(8,9$, and 10) of the final Endcap detector. A carbon fibre structure for holding all services from the endcap silicon detectors was attached to the three disks. Disk 9 was equipped with two TEC sub-structures ("petals"), each holding 17 silicon strip modules distributed in rings 4-7. The pitch of the TEC silicon strip detectors is variable, ranging from 113 to $205 \mu \mathrm{m}$ in these rings. The positions of all silicon modules were final in $r$ and $\phi$ and were shifted by $10 \mathrm{~mm}$ in $z$ to guarantee the final position of the Alignment ring, which was attached with a 10-mm-thick adapter ring on the TEC pillars.

All three sub-structures (TOB, TIB, TEC) were mounted inside an aluminum prototype of the Tracker support tube. With a length of $5303 \mathrm{~mm}$ and an outside diameter of $2400 \mathrm{~mm}$, the prototype had the same dimensions as the actual Tracker support tube. After mounting the substructures in the support tube, all modules were tested again to check that there have been no damages. The modules were cooled using the cooling circuits integrated into the support structures. The temperature of the coolant was about $18^{\circ} \mathrm{C}$ and dry air was passed through the Tracker support tube in order to prevent condensation. Two sets of scintillators, one above and one below the barrel 
Table 1. Modules mounted in the MTCC Tracker structures.

\begin{tabular}{|c|c|c|c|c|c|c|c|}
\hline \multirow{2}{*}{$\begin{array}{c}\text { Tracker } \\
\text { Subdetector }\end{array}$} & \multirow[t]{2}{*}{ Layer/Ring } & \multicolumn{2}{|c|}{ Position } & \multicolumn{3}{|c|}{ Module } & \multirow{2}{*}{$\begin{array}{c}\text { Number } \\
\text { of modules }\end{array}$} \\
\hline & & $r(\mathrm{~cm})$ & $z(\mathrm{~cm})$ & Type & Pitch $(\mu m)$ & N. of channels & \\
\hline \multirow{3}{*}{ TIB } & Layer 2 & $32.2-35.6$ & $2.9-60.6$ & $r \phi$ & 80 & 768 & 15 \\
\hline & & & & stereo & 80 & 768 & 15 \\
\hline & Layer 3 & $40.3-43.4$ & $7.5-59.4$ & $r \phi$ & 120 & 512 & 45 \\
\hline \multirow{2}{*}{ TOB } & Layer 1 & $59.1-62.9$ & $8.9-98.6$ & $r \phi$ & 183 & 512 & 12 \\
\hline & Layer 5 & 94.6-98.4 & $8.9-98.6$ & $r \phi$ & 122 & 768 & 12 \\
\hline \multirow{5}{*}{ TEC } & Ring 4 & 56.2 & $270-278$ & $r \phi$ & $113 / 143$ & 512 & 7 \\
\hline & Ring 5 & 67.7 & $267-274$ & $r \phi$ & $126 / 156$ & 768 & 5 \\
\hline & & & & stereo & $126 / 156$ & 768 & 5 \\
\hline & Ring 6 & 81.9 & $270-278$ & $r \phi$ & $163 / 205$ & 512 & 7 \\
\hline & Ring 7 & 99.2 & $268-275$ & $r \phi$ & $140 / 172$ & 512 & 10 \\
\hline
\end{tabular}

layers, provided a trigger on cosmic muons, allowing to check synchronization procedures and measure the signal for minimum ionizing particles. Finally the tube was carefully transported to the assembly hall at SX5 with a maximum allowed shock of $0.1 \mathrm{~g}$. The tube was inserted into the solenoid using the final insertion tools.

\subsection{Detector Control and Safety Systems}

In the MTCC, the Tracker Control System (TCS) [ [. 5] had to control 72 CAEN power supply channels and to deal with the signals from 40 environmental sensors (temperature and humidity). The dataflow is shown in figure 2. The environmental sensors were directly connected to Programmable Logic Controllers (PLC), which formed the core of the autonomous hardware Tracker Safety System (TSS) [5]. They interlocked the power supplies based on high temperatures, cooling system failures, and global Detector Safety System (DSS) [⿰] w warnings. Through access to TSS information, the TCS could react to alarm situations by switching off the power supplies in a controlled manner. The TCS was fully embedded in the global Detector Controls System [6], following all necessary guidelines. It controlled the CAEN EASY power supply system [П], evaluating information from the environmental sensors and monitoring the state of the cooling plant. Some initial reading and handling of environmental data from the Detector Control Units (DCU) on the front-end hybrid chips was also implemented.

The control software was developed in the framework of version 3.1 of the commercial Supervisory Control and Data Acquisition software PVSS (Prozessvisualisierungs- und Steuerungssystem by ETM [8]). This software was extended in a common LHC framework. The software allowed the setting of the following items: automated control actions, handling of datapoints, management of the communication with the hardware and treatment of alarms, and warning and error messages. The software further provided data archiving to an Oracle database and value trending functionalities.

The tracker control and safety system can be described by a Finite State Machine (FSM). According to the hardware status and user-issued commands, the system can be in several finite states characterized by the presence of the hierarchically organized sub-components, as shown in 


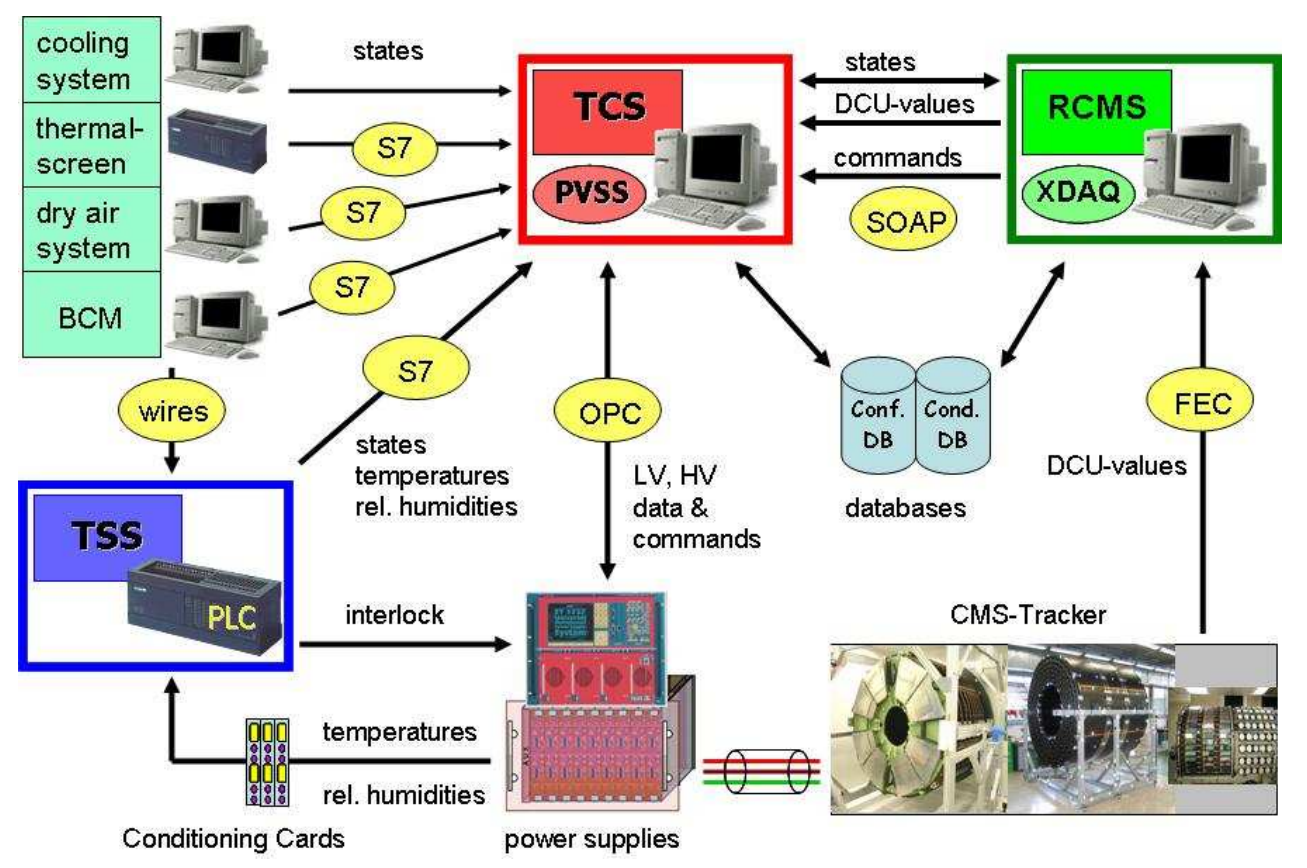

Figure 2. Dataflow in the TCS

figure 3 . The possible transitions between the different states are limited and exactly defined. The MTCC FSM, whose architecture was close to the final one, also controlled the switching sequences that allowed the system go through several intermediate states. This is necessary as parts of the system have to be switched on before others for both safety and technical reasons.

The data of the detector environmental sensors were routed by the standard LIC and PLC cables to the CAEN system and then re-routed from the back of the power supplies to condition cards, forming the input signal to the PLC system. The PLC information was passed to the TCS by the proprietary Siemens S7 driver [9]. The essential communication between the TCS and the power supplies was based on the Microsoft OPC (OLE for Process Control) standard [10], while the communication between the Run Control and Monitoring System (RCMS) [11] and the TCS was guaranteed by PSX (PVSS SOAP eXchange) [12], an application based on the SOAP (Simple Object Access Protocol) protocol [13]. The CAEN and PLC data were written to the database only when there were significant changes from the previous measurements.

The MTCC was an important test of the TCS concept for the final system. In the beginning, problems arose due to hardware changes, bugs and, in general, the complexity of such a large system with many interactions. All of the problems were solved during the initial phase of the MTCC operation at SX5. The run also demonstrated that the TSS and the PLC combination responded to safety conditions as expected. The MTCC represents the first CMS DCS set-up in which archiving to a database was successfully implemented. The experience with real life hardware uncovered some problems in archiving that might have otherwise been missed. The expectation is that these will be addressed in future software releases. Similarly, the experience pointed out areas in which the user display panels could be improved. The successful interaction between the TCS and the Tracker hardware in the MTCC gives confidence that the TCS will function correctly when the full Tracker is installed. 


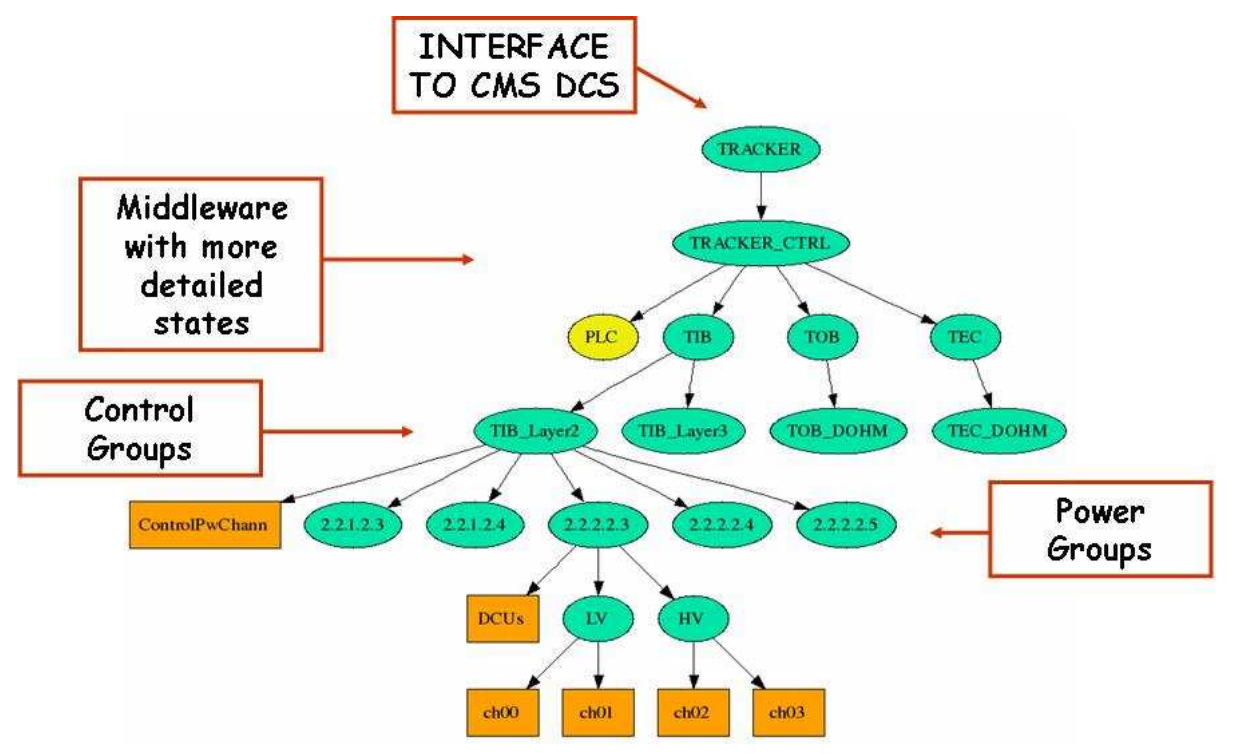

Figure 3. FSM Hierarchy

\subsection{Detector Readout System}

The signals from the silicon sensors are amplified, shaped, and stored by a custom integrated circuit, the APV25 [14] (hereafter shortened to APV). Upon a positive first level trigger decision the analogue signals of all channels are multiplexed and transmitted via optical fibers [15] to Front End Driver (FED) boards [16] where the analogue to digital conversion takes place. The Tracker FEDs can either output one value per channel, in which case they are said to work in Virgin Raw mode, or perform zero suppression. In the latter case, previously uploaded pedestals and noise values for each individual channel are used. The firmware algorithm implemented within FPGA devices perform pedestal and common-mode noise (an event-by-event fluctuation of all channels of one APV) subtraction before identifying channels above a given signal-to-noise threshold. A threshold of two was applied for sets of two or more contiguous strips and of five for isolated strips. The Tracker FEDs can also work in two alternative modes, the so called Scope and Processed mode, which are not described in this note.

The Tracker control system consists of control rings that start and end at the off-detector Front-End Controller (FEC) boards [17]. Slow-control commands, clock and Level-1 triggers are distributed via digital optical links to Digital Opto-Hybrids (DOH) [18], which perform optical-toelectrical conversion before the control signals are distributed to the front-end electronics.

The readout and control electronics setup for the MTCC is summarized in table 2 .

A series of procedures, already used in various tracker integration and beam test setups, are needed to configure, synchronize and calibrate the Tracker readout system. They are primarily concerned with the configuration of the APVs, the other on-detector ancillary chips and the offdetector FEDs. The term commissioning is used throughout the text to indicate such operations. Commissioning is carried out in dedicated calibration runs taken prior to the actual cosmics runs. These runs are normally performed using a local data acquisition system based on the VME readout of the FEDs. 
Table 2. Tracker MTCC readout electronics. An mFEC is a mezzanine FEC, representing one eighth of a full FEC.

\begin{tabular}{|c|r|r|r|}
\hline $\begin{array}{c}\text { Tracker } \\
\text { Subdetector }\end{array}$ & FED & mFEC & APV \\
\hline TIB & 2 & 2 & 360 \\
\hline TOB & 1 & 1 & 120 \\
\hline TEC & 1 & 1 & 156 \\
\hline
\end{tabular}

Several improvements were tested for the first time in the MTCC.

- The commissioning applications were controlled using the RCMS framework. This framework allowed both the initialization (distributed processes start-up) and the configuration of applications. A set of predefined configurations was prepared for each of the commissioning tasks: connection scan, timing adjustement, optical gain adjustement, base-line scan, pedestal and noise computation for each channel. In this way non-expert shift personnel could recommission the Tracker on demand. A reduced configuration where the event building and analysis parts were dropped was then integrated in the global Run Control system for use in global data taking.

Eventually the readout synchronization with the Level-1 trigger was adjusted offline using cosmic muons events (section 4.2). This last procedure is currently being automated and integrated in the commissioning procedures.

- A dedicated database, the online tracker configuration database, was systematically used to store optimal values of the parameters resulting from the commissioning tasks. At the start of a cosmics run, when the configuration of the Tracker electronics is triggered by the central RCMS system, these values were retrieved from the database and uploaded in the electronics. An interactive application was also used for accessing and modifying parameters, as needed.

- The implementation of the analysis part of the commissioning procedures in the framework of CMSSW was tested towards the end of the MTCC phase I. The original plan to run these applications in the Tracker local DAQ had to be postponed due to lack of time. The commissioning analysis was performed with a set of standalone XDAQ [19] applications.

\subsection{Trigger, Data Acquisition and Computing systems}

The MTCC was carried out in the SX5 surface assembly hall. In addition to the tracker setup, about $5 \%$ of the final CMS experiment was also instrumented and readout during the MTCC phase I, as follows:

- two final Super Modules of the Electromagnetic Calorimeter (ECAL), corresponding to about $5 \%$ of the final system;

- fifteen Hadron Calorimeter (HCAL) wedges, corresponding to about $10 \%$ of the final system. 


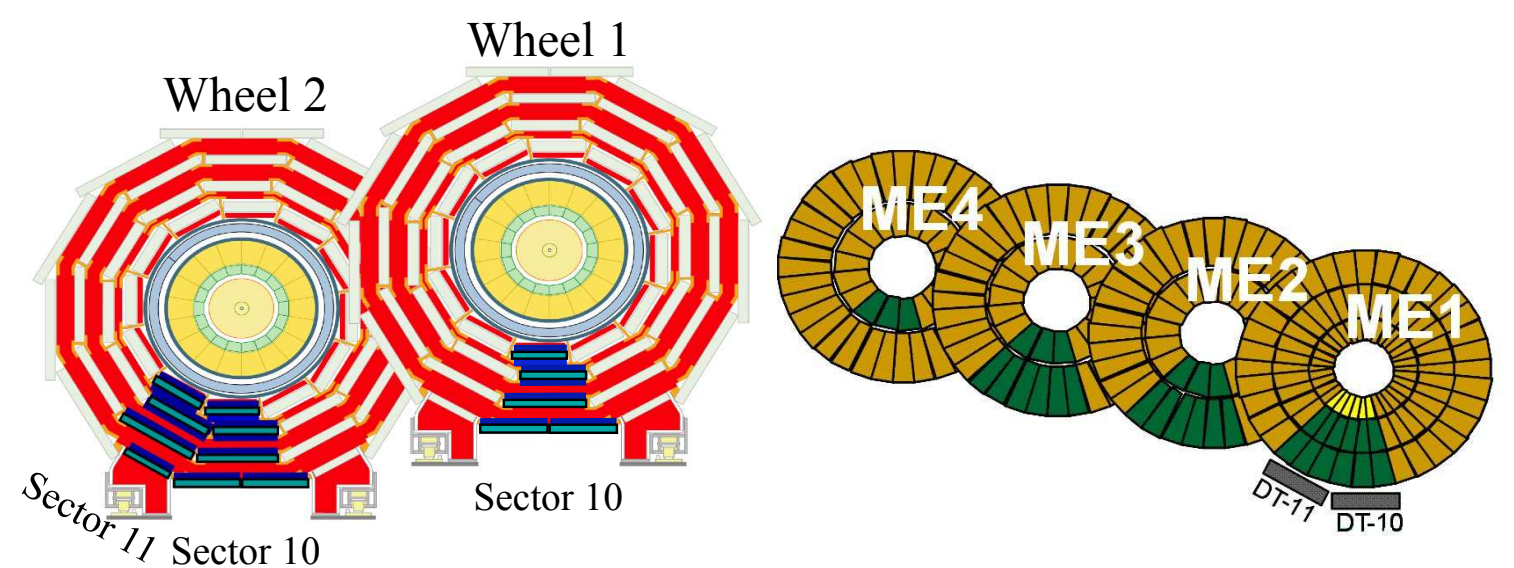

Figure 4. Left: CMS barrel wheels with DT chambers that have been operated at the MTCC. Right: exploded view of the endcap muon disks instrumented with CSC detectors.

- fourteen Drift Tube (DT) chambers and 23 Resistive Plate Chambers (RPC), instrumenting part of the muon barrel region: two sectors of wheel $\mathrm{YB}+1$, covering about 60 degrees in $\phi$, and one sector (about 30 degrees in $\phi$ ) of wheel $\mathrm{YB}+2$.

- thirty-six Cathode Strip Chamber (CSC) detectors covering a 60 degree $\phi$ region and the three innermost disks of one of the two muon endcaps. Some CSC detectors were also present in part of the outermost disk, but they did not participate in the MTCC. Similarly, RPC detectors were also present in the Muon endcap region, but they were not used in neither the Level-1 trigger nor in the global readout.

A schematic view of the full MTCC setup is shown in figure 7 .

The Level-1 trigger signal was mainly derived from the muon detectors. The fast signal produced by the trigger electronics of these detectors was routed to a central system, similar to the one in the final experiment, which handled the trigger logic and distributed a global trigger signal to all detectors for data readout.

The central Level-1 trigger system was receiving signals at its inputs from up to six different sources at a time.

- DT signal. Among the different configurations used, the most important for the Tracker were:

- inclusive, where at least 2 chambers in the same sector and wheel with track stubs are required;

- pointing, which is as above, but with constraints on the $\eta$ segments of the track stubs so that the latter are aligned as to point to the center of the detector.

- CSC signal, when one track stub is found in any chamber with hits in at least 4 out of 6 layers.

- RPC signal which requires hits in 5 out of 6 planes, either in wheel YB+1 (RPC1) or in wheel $\mathrm{YB}+2$ (RPC2) or in any of the two wheels but aligned as to point to the centre of the detector (RPCTB). 
Table 3. Trigger configurations used during the MTCC phase I. The trigger rate corresponding to each configuration as well as the fraction of events with hits in at least three tracker layers are reported.

\begin{tabular}{|l|c|c|}
\hline $\begin{array}{l}\text { Trigger } \\
\text { type }\end{array}$ & $\begin{array}{c}\text { Event } \\
\text { rate }(\mathrm{Hz})\end{array}$ & $\begin{array}{c}\text { Fraction with } \geq 3 \text { hits } \\
\text { in the Tracker }\left(\times 10^{-3}\right)\end{array}$ \\
\hline DT pointing & 10 & 2.3 \\
DT inclusive & 40 & 0.5 \\
CSC & $40-60$ & 0.03 \\
RPC1 & $15-20$ & 0.7 \\
RPC2 & $15-20$ & 0.06 \\
RPCTB & 10 & 1.2 \\
\hline
\end{tabular}

- HCAL signal, corresponding to the coincidence of signals from the upper and lower part of the active HB detectors.

In both DT and RPC Level-1 trigger signals the direction of the incoming muon is used and therefore it is possible to select those muons approximately pointing to the tracker region. The observed rates for the six muon-detector-based triggers are shown in table 3 along with the fraction of events in which there were also hits in at least three of the tracker layers. A detailed description of the Tracker event selection is given in section 3.4. Even with the pointing triggers, only a very small fraction of all triggered muons crossed the tracker.

All subdetectors were readout in the global DAQ of CMS. During the MTCC the tracker was readout for the first time within the central data acquisition system. The readout of the Tracker FEDs via their fast serial link SLINK [20] to the global DAQ receiver FRL cards [21] was tested well before the start of the MTCC at the CMS Electronic Integration Centre (EIC). A full crate of FRL boards was available to validate FEDs, the SLINK transmitter and the cables before their final integration. The MTCC test itself was comparatively small, with only four FEDs connected to the central DAQ. The full trigger loop was also validated before MTCC at the EIC using the LTC [22], TTCci [22] boards, the veto system on the FMM [22] and the APV Emulator [22]. During the global data taking the only observed problem was a spurious state lock-up of the SLINK transmitter when the acquisition was stoped abruptly. This problem was reproduced with EIC setups and has been solved after the MTCC.

The DCS, DSS and the software applications that control the Tracker FEDs were also integrated in a central RCMS system. Events were assembled online by the global DAQ and stored on disk. In the first half of the operational period, the global DAQ had a reduced output bandwidth and, as the Tracker was taking data in raw mode, the maximum allowed event rate on disk was about 30 $\mathrm{Hz}$ (with only Tracker and DT read out). Furthermore, none of the pointing triggers were tested in this initial period. In order to maximize the number of triggered muons crossing the Tracker, only the inclusive DT trigger based on sector 10 and wheel $\mathrm{YB}+1$ was used.

The DT and RPC pointing triggers yield almost the same number of good muons and a factor four lower rate compared to the inclusive DT and RPC trigger rate. During the commissioning of the sub-detectors, as zero suppression was not always applicable to the data, the event rate was limited by the bandwidth for writing to disk. Therefore the use of the pointing triggers was very 
important for the tracker. In the final global DAQ configuration, however, almost all subsystems were taking data in zero suppressed mode and it was possible to write up to 200 events per second to disk and the pointing trigger was no longer required to reduce the rate. For this reason, only half of the events in the final data set were taken with the DT pointing trigger while the other half were taken with the inclusive trigger.

The raw data were transferred from CERN T0 centre to FNAL T1 centre using the final CMS computing tools [23]. At FNAL, the raw data files were converted into a CMSSW-compatible format and the initial reconstruction was performed. The FNAL Remote Operations Center (ROC) played a significant rôle in the automated processing of the raw data, contributing to quasi-online data monitoring and fast offline data analysis. Further reconstruction passes were done at FNAL to include the tracker alignment and improved tracking algorithms. All output files were transferred back to CERN and stored in CASTOR.

\section{Offline software}

The MTCC event reconstruction, event selection, data quality monitoring, simulation, and data analysis were performed within the framework of CMSSW. As reported in the previous section, the tracker commissioning software had not been fully ported to the CMSSW environment at the start of the MTCC. For this reason an older version of the software was used for the vast majority of the tests to commission the tracker.

A CMSSW application consists of a set of software plug-in modules which are executed at every event. The list of plug-ins and their order of execution is specified in the job configuration file. Event data processing plug-ins communicate with each other by exchanging information through a single data structure referred to as the "Event". Applications can run in a completely transparent fashion in both offline and online applications. The only difference resides in the "input" plug-in, which is responsible for putting the primary input data in the Event. When running online, the input plug-in puts detector raw data received over the network from the data acquisition system in the Event. When running offline the input plug-in reads from disk detector raw data and/or higherlevel reconstructed data, which may be present on the data files, and puts it in the Event for further processing.

A CMSSW application requires the availability of non-event data such as alignment constants, magnetic field values, and calibration data. This information is stored in a dedicated "offline" database, to which CMSSW provides a uniform software interface. Two offline databases are maintained [24]: the first one, ORCON, is used by applications running on the online data acquisition farm whereas the second, ORCOFF, is used by offline jobs running on the GRID.

\subsection{Reconstruction}

All of the tasks listed above rely on the tracker event reconstruction plug-ins. A reconstruction plug-in gets the required input data from the Event and puts higher-level reconstructed data back into it. Based on the raw data, the following objects can be produced.

- Digis: pedestal-subtracted and zero-suppressed ADC counts for individual strips. 
- Clusters: groups of adjacent strips whose associated ADC counts pass a set of thresholds. The thresholds depend on the noise levels characterizing the strips of the cluster.

- RecHits: estimated position and error of charged particle crossings.

- Tracks: sets of RecHits compatible with a particle trajectory in the detector. Each track also contains momentum and error matrix information from the track fitting procedure, evaluated at the innermost and outermost layers.

\subsubsection{Local reconstruction}

Digis are produced by the first plug-in in the reconstruction chain, Raw2Digi. The Raw2Digi plugin unpacks the blocks of raw data produced by each FED and groups the Digis of each detector module together. Each group of Digis is associated with a unique integer which encodes the location of the module in the tracker mechanical structure. The assignment of Digis to detector modules can only be performed if the connections between detectors and FED channels are known. All connections are automatically detected by the tracker commissioning software in dedicated runs and stored on the tracker online configuration database. This cabling information needs to be transferred to ORCON and ORCOFF in order to be used by the Raw2Digi plug-in. For this reason, a special Online-to-Offline $(\mathrm{O} 2 \mathrm{O})$ application was developed and used at the MTCC.

Cluster reconstruction is the task of the next reconstruction plug-in in the analysis sequence. Clusters are reconstructed by searching for a seed strip with a signal-to-noise ratio $(\mathrm{S} / \mathrm{N})$ greater than 4. Nearby strips are included in the cluster if their $\mathrm{S} / \mathrm{N}>3$. The total signal size of the cluster must exceed five times the quadratic sum of the individual strip noises. The signal of each channel is currently taken as the raw ADC counts stored in the corresponding Digi. In the actual experiment, this signal is expected to be corrected for the gain of the channel. The gain calibration at the MTCC is discussed in section 5.5 .

Cluster reconstruction requires access to the noise level of each individual tracker channel as well as a map of bad channels. In addition, if the tracker is read out in a non-zero-suppressed mode, pedestal values are also necessary. Pedestals, noise and the bad channel map are normally computed during commissioning runs and stored in the tracker online configuration DB so that they can be used to configure the readout electronics. Based on the model for handling cable data, another dedicated $\mathrm{O} 2 \mathrm{O}$ application was developed to transfer this data to ORCON and ORCOFF. This $\mathrm{O} 2 \mathrm{O}$ application performs some data manipulation beside the simple data transfer. Indeed, the values stored in the tracker configuration DB are arranged in groups, each corresponding to the channels readout by a pair of APV chips. On the other hand, cluster reconstruction must be performed at the module level. The $\mathrm{O} 2 \mathrm{O}$ application accesses the data on the tracker configuration $\mathrm{DB}$, rearranges it, transfers it to the ORCON and ORCOFF databases and finally sets an appropriate "interval of validity" (IOV). The interval of validity for any given data set is defined as the range of consecutive events to which it applies. Typically, the IOV of pedestals, noise, and bad strips spanned a few days of data taking at the MTCC. The IOV of the cabling object was set to last for the full duration of the MTCC.

Every cluster is associated with a RecHit by a dedicated CMSSW plug-in. The RecHit position is determined from the centroid of the signal heights. The position resolution is parameterized as a quadratic function of the projected track width on the sensor in the plane perpendicular to the 
strips [25]. Whenever the modules are operated in a magnetic field, a parameterization of the Lorentz shift is used for all modules to correct the RecHit position. In the actual experiment, the Lorentz angle of each tracker module is expected to be measured from the data. The algorithm for measuring the Lorentz angle has been tested for the first time on real data at the MTCC and its results are presented in section 5.6 .

\subsubsection{Track reconstruction}

In CMSSW all track reconstruction algorithms use a Kalman filtering technique [26] and involve three basic steps:

1. Seed creation: A seed is a minimal set of RecHits that are compatible with a particle trajectory and with which it is possible to give a first estimate of the track parameters.

2. Pattern recognition: This step results in the making of collections of RecHits that are compatible with a particle trajectory. The procedure starts by propagating each seed track state in succession to all tracker layers that have not contributed to the seed with RecHits. After each propagation, which takes into account magnetic field bending, energy loss in the material, and multiple scattering, RecHits found on the layer are tested for compatibility with the propagated state. A new track candidate is created for each compatible RecHit and the track state is updated according to the Kalman Filter formalism with information carried by the new RecHit. The procedure is repeated until either the last layer of the tracker is reached or a stopping condition is satisfied.

3. Track fitting: Track fitting and smoothing final track parameters for all tracker layers are computed in this step, though only those at the innermost and outermost layers are retained and stored on disk. The Kalman filter is repeated for each candidate track in both directions: from inside out and outside in. The predicted results from both filters are combined to yield optimal estimates of the parameters at the surface associated with each hit. In this step, all RecHit positions are also updated using the information of the track incident angle on the layer to which the RecHit belongs. More details on track reconstruction algorithms can be found in ref. [25].

Two different track reconstruction algorithms have been used on MTCC data: the Cosmic Track Finder and the Road Search algorithm.

The Cosmic Track Finder [27] was developed specifically for cosmic tracking. This algorithm is a variation of the Combinatorial Track Finder (CTF) [27]. The standard seed creation algorithm used by the CTF is not as yet appropriate for tracks that do not point to the interaction vertex, hence the algorithm was not run on the MTCC data set. The Cosmic Track Finder uses any pair of RecHits from different layers to build a seed. This choice does not decrease the speed of the track reconstruction step because cosmic events yield a considerably lower number of RecHits than p-p events. For the MTCC setup, the Cosmic Track Finder requires that the two RecHits of a seed are either on the two innermost layers or on the two outermost ones. In the TIB region, where an overlap of about $5 \%$ between the modules on the internal and external rings exists, RecHits can be 
used to build a seed even if they come from the same layer, but under the condition that one RecHit comes from the internal ring and the other from the external one.

Pattern recognition in the Cosmic Track Finder begins by ordering all RecHits with respect to the vertical direction, that is, according to the global $y$ coordinate of the RecHit in the CMS reference system. Then the algorithm attempts to add to the candidate track the RecHits in the order defined by the previous sorting procedure. All RecHits in the given layer are tested for compatibility. The compatibility of the hit with the propagated trajectory is evaluated using a $\chi^{2}$ estimator. The maximum allowed $\chi^{2}$ value is an adjustable parameter in the algorithm. At the end of this phase several trajectories are still valid, but only one is retained since generally only one track per event is expected. The results shown in this document have been obtained by choosing the best trajectory according to the following criteria:

- largest number of layers with hits in the trajectory.

- largest number of hits in the trajectory.

- smallest $\chi^{2}$ value.

The Road Search (RS) algorithm was also run on the MTCC data. The algorithm is characterized by a navigation scheme based on pre-defined groups of silicon modules, called "Roads". All modules in the same $(r, z)$ region (in the CMS reference system) are first grouped into "Rings". Pairs of Rings are then chosen to serve as "Road seeds". A Road is finally made up of all Rings intersected by a straight line going through the two Road seeds in the $(r, z)$ plane. A track seed in the RS algorithm is built out of two RecHits found in modules belonging to the "Road seed" Rings of a given Road. Creation of the track seeds starts by looping over the Road seeds of all pre-defined Roads. Pairs of RecHits are searched in the corresponding detector modules. A field-dependent constraint on the $\Delta \phi$ between the RecHit in the inner Road seed and the RecHit in the outer Road seed ensures that only sensible combinations are retained for further processing. Pattern recognition proceeds as in the Cosmic Track Finder, by successive extrapolations, but only the RecHits in the Road in which the seed was found are checked for compatibility with the track extrapolated state. The track fitting step in the RS algorithm is the standard one used by the CTF algorithm. Unlike the Cosmic Track Finder, all reconstructed tracks are retained by the RS algorithm. The standard algorithm, which was designed for use in p-p collisions, had to be slightly modified in order to reconstruct cosmic muons. These tracks do not originate from the interaction region and therefore the beam spot constraint, used in making track seeds, had to be loosened. Specific Roads had to be generated for the MTCC. All modules from the TIB layer 2 were chosen to be the Road inner seed, while the outer Road seed can stem from either TOB layer 1 or TOB layer 5. An overview of the inner and outer seed Rings for the MTCC geometry is shown in figure 5. This figure also includes the Road of a possible cosmic ray track. The results of the Road Search algorithm are presented in section 5.2.5.

\subsubsection{Alignment}

The CMS software alignment framework was used to align the tracker with data collected at SX5. The final alignment was obtained using the hits and impact points algorithm (HIP) [28], a speciallydeveloped iterative algorithm. The HIP algorithm is both computationally light and flexible, and 

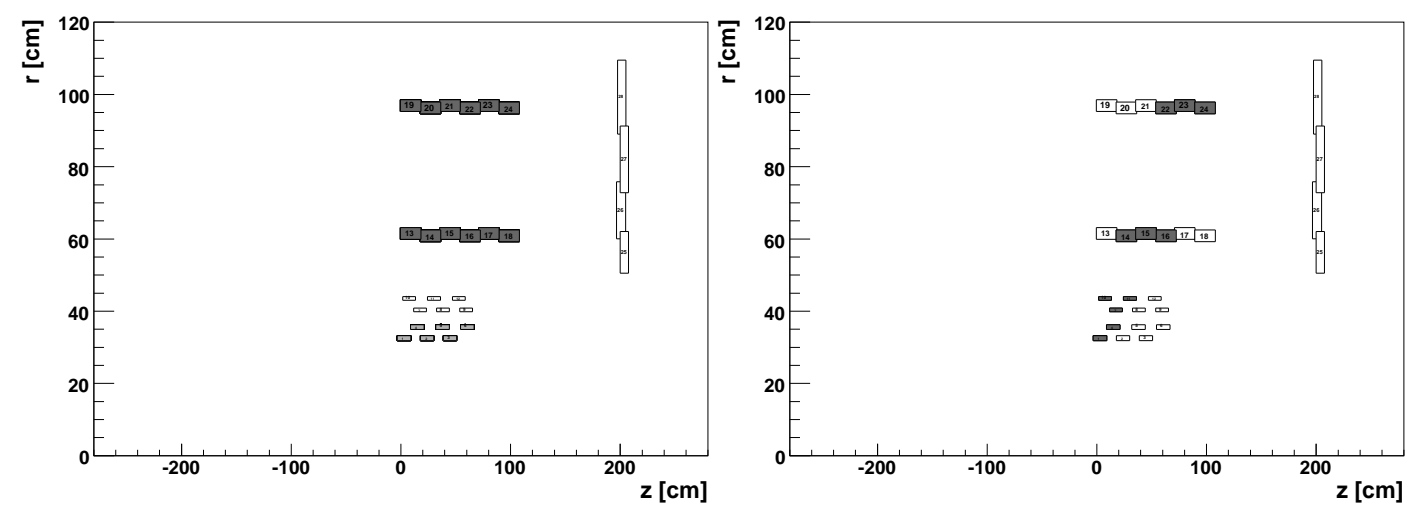

Figure 5. The left plots shows an overview of the inner seed rings (light grey) and outer seed rings (dark grey) as defined for the Road Search algorithm. The right plot shows the road of a possible cosmic ray track.

had been successfully used for the alignment of CMS tracker modules in both testbeam and simulation studies prior to the MTCC.

Construction information was processed to produce initial corrections to the position of the modules (section 5.2.1). These corrections were stored in the offline DB and used as a starting point for the HIP algorithm.

The final alignment corrections computed by the HIP algorithm were also stored in the offline DB. They contain the global corrected positions of all silicon sensors. These data are read in during the track reconstruction step to provide an aligned geometry.

\subsection{Simulation}

The simulation of the MTCC is needed to estimate the rate of muon tracks crossing the triggering detectors and the fraction of these tracks that also cross the tracker detectors. This information is useful to optimize event selection, discussed in section 3.4 and interpret the results of the data analysis. In addition, tuning of the simulation of the tracker detector response can be performed using MTCC data.

In general, the generation of the primary particles in an event and their transport through the material and magnetic field of CMS result in the production of the following simulated tracker data:

- SimTrack: either primary or secondary track.

- SimVertex: either primary or secondary vertex.

- SimHit: entry point, exit point and deposited energy of a particle in the sensitive volume of the tracker detectors.

Every particle produced as a result of an interaction of the particle being transported is in turn transported (and a new SimTrack and a new SimVertex are created) assuming they exceed certain energy thresholds. These values depend on the volume where the interaction takes place and were set so as to provide a balance between the needs for an accurate simulation of the detector response and an acceptable CPU performance.

The cosmic muons have been generated using the CosmicMuonGenerator [29] code. It is based on the CORSIKA program [30], with a distribution parameterization accuracy of about $5 \%$ 

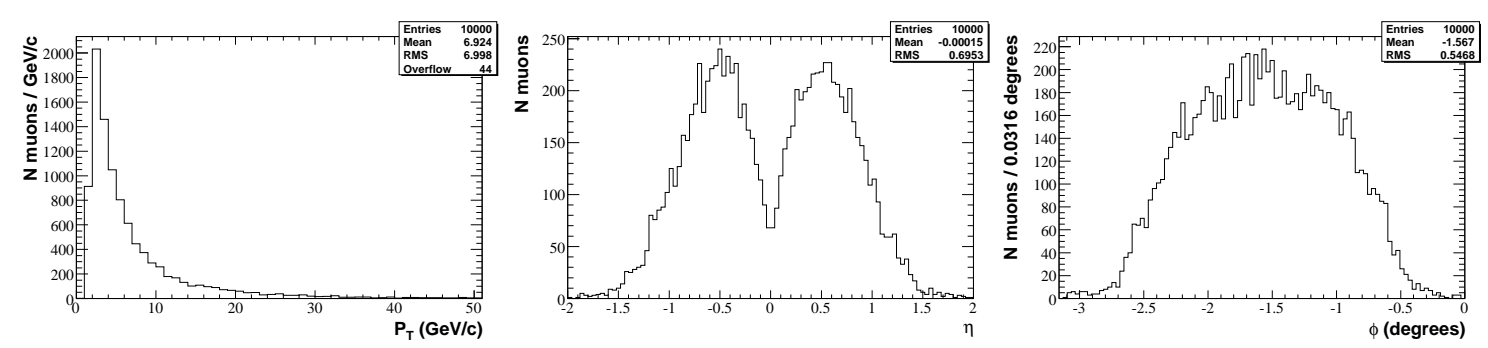

Figure 6. $P_{T}, \eta$, and $\phi$ distributions for cosmic muons at generator level.

for $10-1000 \mathrm{GeV}$ of energy and $0-75^{\circ}$ in zenith distribution (the accuracy is $10 \%$ in the ranges $2-1000 \mathrm{GeV}$ of energy and $0-88^{\circ}$ in zenith distribution). The edges of the energy spectrum and the angles with respect to the vertical direction can be adjusted by the user. figure 6 shows the $P_{T}, \eta$, and $\phi$ distributions of the generated muons for a sample produced with an energy spectrum between 2 and $10000 \mathrm{GeV}$ and with an azimuthal angle between 0 and 88 degrees.

In the most general configuration, simulated cosmic muons start at the outer surface of the CMS detector and are then propagated through the MTCC detector elements until they hit a target surface. The dimensions of the target surface can be adjusted by the user in order to improve the generation efficiency. Only those events where the muon reaches the target surface are then kept and passed to the simulation phase. For comparison with the MTCC data two different data sets are needed, field on and field off. The small dimensions of the MTCC tracker (roughly corresponding to: $R_{\text {tracker }}=1.2 \mathrm{~m}, L_{\text {tracker }}=1.2 \mathrm{~m}$, with an opening azimuthal angle of $92^{\circ}$ for the rods of the TIB and TOB) imply a very small rate for contained muons tracks. An optimization of the generation efficiency is necessary in order to create Monte Carlo data sets with the same order of magnitude of events with reconstructed muon tracks as in the data in a reasonable amount of time and CPU. There are various parameters that allow to customize the size and shape of the target surface:

- If the parameter MTCCHalf is set to true the generator passes only muons crossing the positive half of the detector to the simulation. This corresponds to the region of the MTCC tracker.

- The length and the radius of the cylinder considered as Target Surface can also be set by hand.

Additional filters are then applied after generation in order to minimize the events passed through simulation or reconstruction.

The events with $\mathrm{B}=0 \mathrm{~T}$ have been produced with $L_{\text {target }}=1.2 \mathrm{~m}$ and $R_{\text {target }}=1.2 \mathrm{~m}$ and with a maximum opening angle of $55^{\circ}$ with respect to the vertical plane. Figure 7 shows a pictorial view of the CMS volume considered as target region in this case.

After the generation stage, the events are passed to the simulation. A filter (SimFilter) is then applied that requires the presence of at least one simulated hits (SimHit) in the tracker. Figure 8 shows the $P_{T}, \eta$, and $\phi$ distributions of the muons selected by the generator level filter. The number of selected events after this filter is $5.9 \%$ of the total generated sample.

Table 4 summarizes the efficiencies of the various filters in the simulations with $\mathrm{B}=0 \mathrm{~T}$. The fraction of simulated events which pass the event selection applied on real data, as explained in 


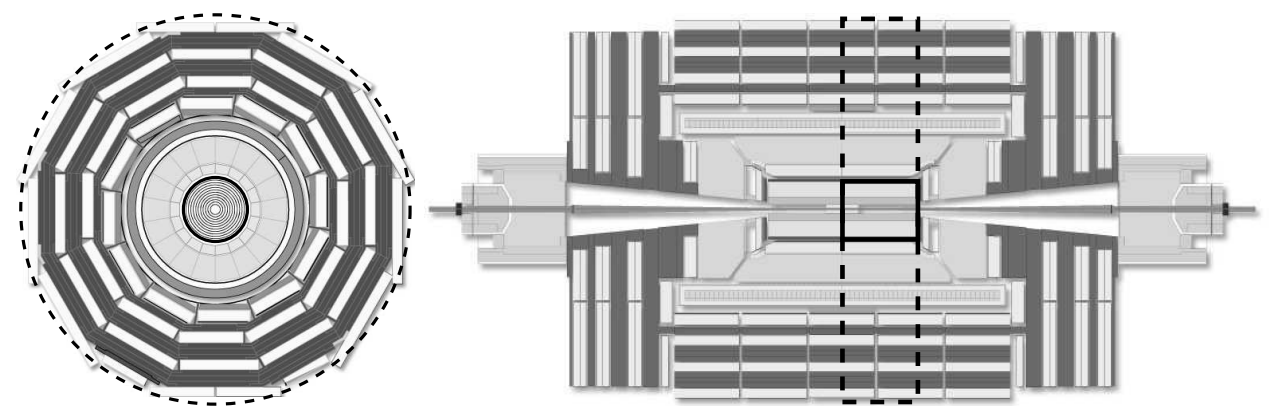

Figure 7. View of the CMS Generator Target region: xy view (left) and yz view (right). Only generated muons that cross the detector in the regions defined by a continous line for the $\mathrm{B}=0 \mathrm{~T}$ and by a dashed line for $\mathrm{B}=4 \mathrm{~T}$ are retained for the subsequent analysis.
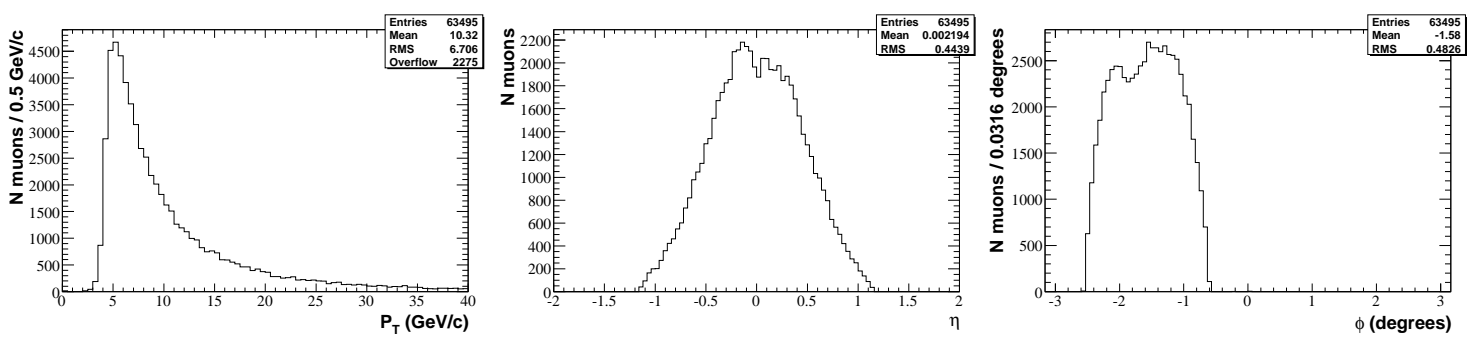

Figure 8. $P_{T}, \eta$, and $\phi$ distributions for cosmic muons in the $\mathrm{B}=0 \mathrm{~T}$ sample.

Table 4. Selection efficiencies of simulated events with $\mathrm{B}=0 \mathrm{~T}$.

\begin{tabular}{|c|c|c|}
\hline Step & Absolute $\varepsilon(\%)$ & Relative $\varepsilon(\%)$ \\
\hline Sim Filter & 5.9 & \\
Event Selection & 0.54 & 9.1 \\
Track Reconstruction & 0.42 & 78 \\
\hline
\end{tabular}

section 3.4, is also shown in this table. The applied filter allows the rejection of most of the events which would not pass event selection. In the last row of the table, finally, the fraction of events where at least one track has been reconstructed by the Cosmic Track Finder is given. This is a rough estimate of the expected number of tracks which should be reconstructed in the selected event sample.

A significant difference arises in the optimization of the generation efficiency for the case of non-zero magnetic field: since the propagation of the muons is done with a straight line, there is an additional inefficiency due to the Lorentz force curvature inside the CMS volume in the case of $\mathrm{B} \neq 0 \mathrm{~T}$. Moreover, reducing the radius target at generator level introduces a strong bias in the $p_{T}$ distribution of the accepted muons. In this case only the length of the Target cylinder can be modified without any bias. The events with $B \neq 0$ have then been produced with a target surface corresponding to a cylinder of $L_{\text {target }}=1.2 \mathrm{~m}$ and $R_{\text {target }}=8 \mathrm{~m}$, corresponding to the outer radius of the CMS detector (see figure 7).

A specially developed filter (GenFilter) which uses the CMSSW Fast Simulation propagator 

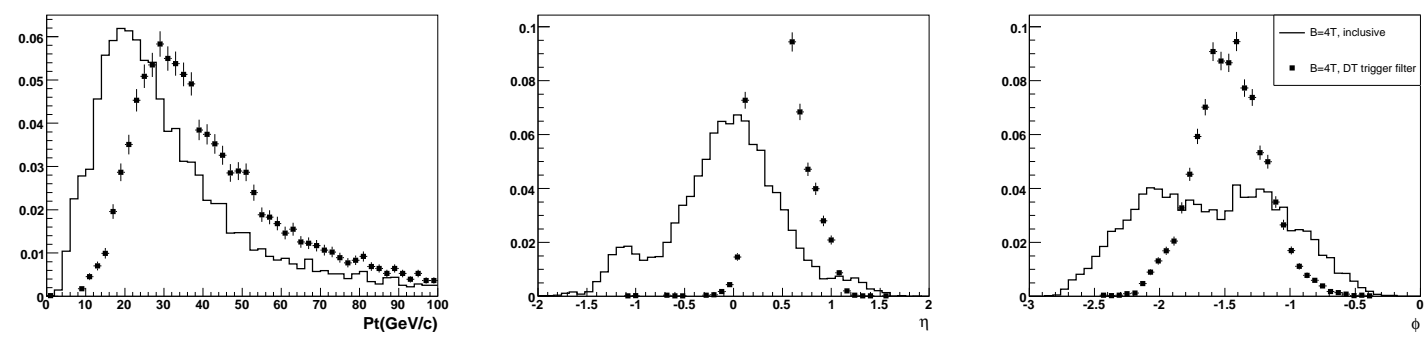

Figure 9. $P_{T}, \eta$, and $\phi$ distribution for cosmic muons in the $B=4 \mathrm{~T}$ sample, before (plain) and after (dots) the DT trigger selection. All distributions are normalized to unity as relative efficiency of the DT filter for events in the tracker can not be measured on data. The distributions are superimposed to approximately show the change in the distributions when a DT trigger is present.

tool was applied to these events before simulation. Muons are propagated through the CMS volume using the magnetic field value in the different regions. These muons were passed to the simulations only if their propagated trajectory intersects the tracker layers in at least three points. The fraction of muons selected by this filter with respect to the total number of generated events was $0.4 \%$. The requirement of at least one SimHit in the tracker on these events has an efficiency of 3.4\%. It should be noted that the generated sample was not produced with $\mathrm{B}=3.8 \mathrm{~T}$, the field value corresponding to the MTCC data, but instead with $B=4 \mathrm{~T}$. This is due to the non-trivial behavior of the CMS magnetic field in the muon detector region, where it cannot be simply rescaled. The CMS magnetic field in the CMS software was parameterized only for the $\mathrm{B}=4 \mathrm{~T}$ case using a map based on the TOSCA software package [31] . A 3.8 T field parameterization was not available at the time of this note.

Table 5 summarizes the efficiencies of the various filters in the simulations with $\mathrm{B}=4 \mathrm{~T}$, together with the fraction of simulated events which pass the event selection and have a reconstructed track, as in table 7 . Figure 9 shows the $P_{T}, \eta$, and $\phi$ distributions of the muons selected by the generator level filter and the SimHit filter for the $\mathrm{B}=4 \mathrm{~T}$ production.

As described in section 2.4, the data were collected using a combination of triggers from the muon system. A detailed simulation of this trigger was not available, but it is interesting to understand the effect of the triggers coming from the DT chambers, the dominant one in the collected data, on the global variables such as muon momentum, eta and phi. A simple filtering based on the presence of SimHits in the DT chambers present in the trigger has been developed and the result is shown in figure 9. Since the DT filter was applied on events where the muon cross at least one tracker module, the fraction of events which are retained could not be measured on data. All distributions of muons variables are therefore normalized to unity to compare their shape in the simulation before and after filter application and with that of reconstructed tracks (figure 54).

Simulation has been used also to test the tracking algorithms. In particular, the angular and momentum resolution as well as the efficiency of the Cosmic Track Finder have been evaluated on the $4 \mathrm{~T}$ Monte Carlo sample. In figure 10 the difference between simulated and reconstructed quantities $\left(P_{T}, \eta\right.$, and $\left.\phi\right)$ are shown for all tracks that have reconstructed hits at least in three layers and for tracks that have reconstructed hits in four layers. In table 6 the resolutions for these track samples are summarized. As expected, the $\eta$ resolution is much worse than the $\phi$ resolution since only one layer provides a precise measurement for the $z$ coordinate of the hit. 
Table 5. Selection efficiencies of simulated events with $B=4 \mathrm{~T}$.

\begin{tabular}{|c|c|c|}
\hline Step & Absolute $\varepsilon(\%)$ & Relative $\varepsilon(\%)$ \\
\hline Gen Filter & 0.39 & \\
Sim Filter & 0.013 & 3.4 \\
Event Selection & $1.1 \cdot 10^{-3}$ & 85 \\
Track Reconstruction & $1.0 \cdot 10^{-3}$ & 91 \\
\hline
\end{tabular}

Table 6. Angular and energy resolution of the cosmic track finder for different quality of the tracker.

\begin{tabular}{|c|c|c|c|}
\hline $\begin{array}{c}\text { Track } \\
\text { quality }\end{array}$ & $\begin{array}{c}\phi \text { resolution } \\
\mathrm{mrad}\end{array}$ & $\eta$ resolution & $\begin{array}{c}P_{T} \text { relative } \\
\text { resolution }\end{array}$ \\
\hline All the tracks & 1.8 & 0.14 & $10 \%$ \\
\hline Three layers & 1.3 & 0.12 & $9 \%$ \\
\hline Four layers & 1.0 & 0.07 & $6 \%$ \\
\hline
\end{tabular}
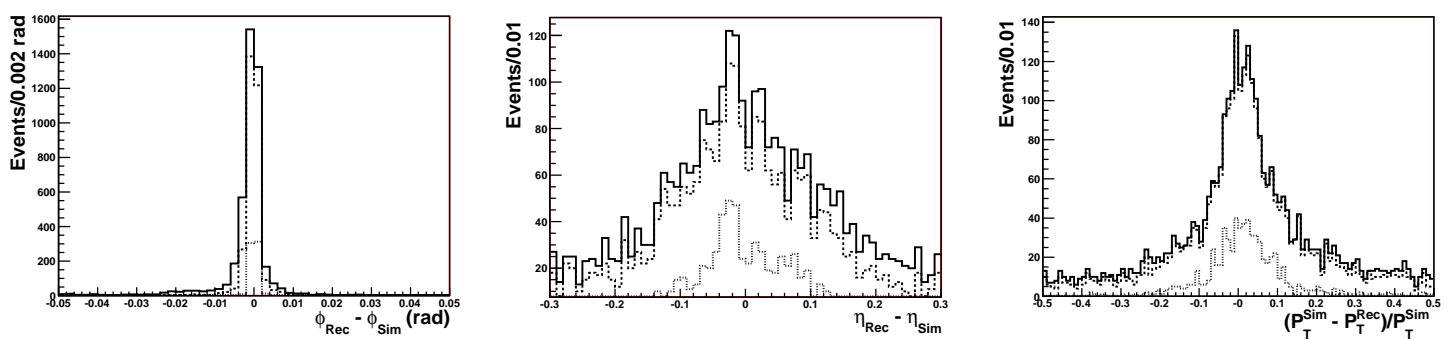

Figure 10. From left to right resolutions of $\phi, \eta$, and the transverse momentum relative resolution. Resolutions are shown for all the tracks (solid), for tracks with hits at least in three layers (dashed) and for tracks that have hits in four layers (dotted).

In order to calculate tracking efficiency, only the events with a seed and at least three reconstructed hits in $r \phi$ modules are considered. Because of overlaps, the tracks can have more than one hit per layer. Reconstructed hits must be correctly associated to a simulated hit created by the muon to be counted. The fake rate has been calculated with the complementary sample (events with fewer than three hits correctly associated).

In figure 11, the efficiency is shown as a function of transverse momentum and for three different track qualities. The estimated fake rate is about $0.2 \%$.

\subsection{Data Quality Monitoring and visualization}

Production of histograms for Data Quality Monitoring (DQM) [32] purposes was performed in special CMSSW plug-ins that have read access to the Event. Event data can be either read from disk or over the network (typically from an online DAQ system node). In the former case, the plug-ins are executed in a standalone CMSSW application, and the DQM is said to run in offline mode. In the latter case, the CMSSW shared libraries containing the plug-ins to be executed are 


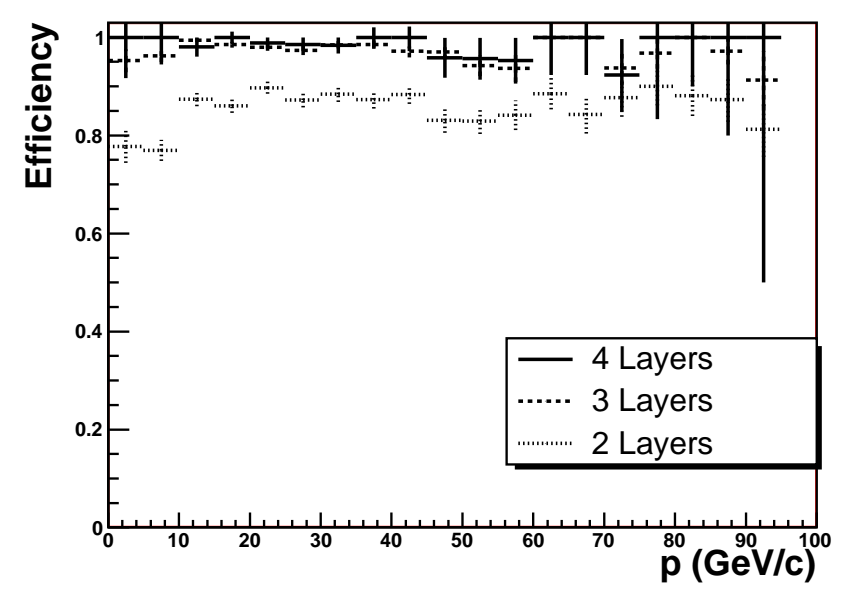

Figure 11. Tracking efficiency for all the tracks with hits correctly associated in at least two, three and four layers. Tracks with hits in two layers only still have at least three hits, one of which is in an overlap region of modules belonging to the same layer.

linked with an XDAQ application and the DQM is said to run in online mode. In either case, the application that produces the DQM histograms is referred to as the "DQM source". At the end of the job or run the DQM source can optionally save the histograms to disk (in Root file format).

The histograms produced by the DQM source fall into the following categories:

- Distributions of local quantities (typically one histogram per detector), such as occupancy, cluster charge and the number of strips making up a cluster.

- Distribution of global quantities, such as number of tracks per event, track pseudorapidity $(\eta)$, azimuth $(\phi)$, momentum $(p)$, transverse momentum $\left(p_{T}\right)$ and residuals between the fit and the actual RecHit positions.

- Event selection related histograms: distribution of the variables on which the event selection is based, number of events selected.

However, as the number of detector modules increases, summary histograms based on average values from individual module distributions take on an increased importance. In the tracker DQM software package, summary histograms are created by a separate XDAQ application, called the "DQM client", which runs a set of dedicated CMSSW software plug-ins. The DQM client receives over the network the histograms produced by the source application, processes the information contained in these histograms, and produces average quantities that are displayed in a selected set of histograms. The DQM client can write to disk the summary histograms it produces and the primary histograms received from the DQM source. The DQM client comes with an interactive Graphic User Interface (GUI) which allows the user to subscribe to the histograms produced by the DQM source, to create summary histograms, to view these histograms and to save them to disk. All these operations can be performed in real time.

Multiple DQM sources can concurrently send the same set of histograms to a DQM client. This mode of operation allows the rate of processed events to be increased. 
It is also possible to execute the actions performed by the DQM source and DQM client in a single application, either offline or online, by using a special job configuration. This application however does not allow the graphical interface to be used.

At the MTCC, the main mode of operation was offline without any DQM client. At the end of each run an offline DQM source was run by the shift personnel on the data files produced during the run. The resulting histograms were inspected and actions taken in case of anomalies. Only at the very end of the data taking period was the DQM run in online mode. In the first instance a DQM source was run on a dedicated node that was receiving from the Storage Manager application events accepted by the DAQ filter farm. Then, the DQM source was run on one of the nodes of the DAQ filter farm and processed Level-1 Trigger accepted events. In both cases, only a simple DQM client could be run. The latter allowed just navigation through the primary histograms produced by DQM source to be performed.

Event-display functionality was provided by the IGUANACMS [33] toolkit included in CMSSW.

\subsection{Event selection}

Event selection is fundamental both for synchronizing the tracker readout with the Level-1 Trigger signal (see section $\bigoplus$ ) and for producing optimal data samples for the analyses. As the angular acceptance of the muon chambers is much larger than that of the tracker volume, among all the triggered cosmic muons only very few were expected to yield a signal in the tracker modules. From simulation studies it was expected that the fraction of triggered events crossing at least one MTCC tracker module be of the order of $10^{-3}$. As a consequence, only a small fraction of the hits in the tracker are due to muons, while the majority is a background due to the electronics noise. Selection of interesting events must therefore be highly rejective.

The request of a track to be reconstructed can certainly provide the necessary rejection, but it may result in low efficiency and unexpected biases, especially in the first phase of the MTCC where the detectors and their alignment were not fully understood. For this reason a simpler and more robust event selection algorithm was developed to select events with a muon in the tracker barrel layers. The algorithm is based upon the presence of clusters in at least three out of the four different TIB and TOB layers. Clusters must also pass a charge threshold of 25 ADC counts (compared to an expected most probable value between 100 and 150 ADC counts) in order to be considered.

Unfortunately, because of the specific MTCC tracker layout, triggered muons could not cross simultaneously TEC modules and any other TIB or TOB layer. Therefore, for TEC studies, events that have physical clusters in multiple layers are not available. A special event selection, which required just one TEC hit in the event, was used. All other cuts on the data were performed later in the analysis. With this selection in place the number of events that were suitable for a dedicated TEC analysis was reduced by roughly $95 \%$ compared to all events taken.

Table 7 presents an overview of the different data taking periods, separated by changes of the magnetic field value. For each data set, the total number of events and the number of events retained for TIB+TOB reconstruction output and TEC reconstruction output are given.

Most of data samples used in the analysis were taken in the period from August 23-29, 2006. During this period, almost no changes to the detector system or the data acquisition system were allowed to ensure that the data taking would be as trouble-free as possible and the data would be 
Table 7. Overview of the MTCC data samples used for the various analyses.

\begin{tabular}{|c|c|c|c|c|}
\hline Data Taking Period & B Field [T] & \#Events & \#Events (TIB+TOB) & \#Events (TEC) \\
\hline August 23-25, 2006 & 0.0 & 7407233 & 4342 & 145176 \\
August 26-28, 2006 & 3.8 & 13765676 & 3953 & 251565 \\
August 28, 2006 & 4.0 & 1715550 & 688 & 30721 \\
August 28-29, 2006 & 0.0 & 3984939 & 2483 & 82239 \\
\hline
\end{tabular}

consistent from the perspective of the offline analysis. Several runs were recorded with the magnet off and with the magnet on at field values of $3.8 \mathrm{~T}$ and $4 \mathrm{~T}$. Requiring that the tracker system is in global readout, a total of about 120 runs remain for tracker-specific analyses in this period.

In addition to these global cosmic runs, several runs consisting of about 100k events each were taken with non-optimally configured readout system during the period when the tracker readout was being synchronized with the global Level-1 Trigger signal.

\section{Detector commissioning}

As anticipated in section 2.3, prior to the data taking, several commissioning tasks had to be performed in order to correctly configure the readout electronics.

Commissioning tasks at the MTCC were initially performed by experts and then also by shifters.

\subsection{Configuration of readout electronics}

First of all, a list of active modules and corresponding FED channels is needed. All analog optohybrid $(\mathrm{AOH})$ lasers are then switched on and off sequentially, while the signals at the inputs of the FEDs are checked. In this way the mapping between the detector elements and the FED channels is determined automatically.

Once the cable map has been determined the APVs are then all aligned in time at the FED. This synchronization is done using the tick mark signal, which produced by the APVs every 70 clock cycles, i.e., every $1.75 \mu$ s. During this procedure the FED samples signals at the full clock frequency in Scope mode. After each DAQ cycle the delay for the APV tick mark is increased by $1 \mathrm{~ns}$ and the measurements repeated. Given the $40 \mathrm{MHz}$ clock frequency it corresponds to an effective FED sampling of $960 \mathrm{MHz}$. The rising edge, $t_{R}$, is measured by the time corresponding to the largest increase in signal, as illustrated in figure 12. Final delay values are then written on the front-end hybrids.

Following the time alignment, a gain value is determined for each $\mathrm{AOH}$. Only four possible gain values can be set in the $\mathrm{AOH}$, allowing a certain amount of gain equalization. The one that results in the tick mark height closest to 640 counts is chosen. The tick mark height is taken to be the difference between the flattop and the baseline of the FED sampling profile. To avoid the use of values from the initial overshoot of the rising edge, the sampling point is taken to be $t_{R}+15 \mathrm{~ns}$ (figure 12).

The height of the tick mark sets the dynamic range of the analog signal from the APV. In the final step of the APV configuration the average pedestal value is adjusted to be about $1 / 3$ of the 


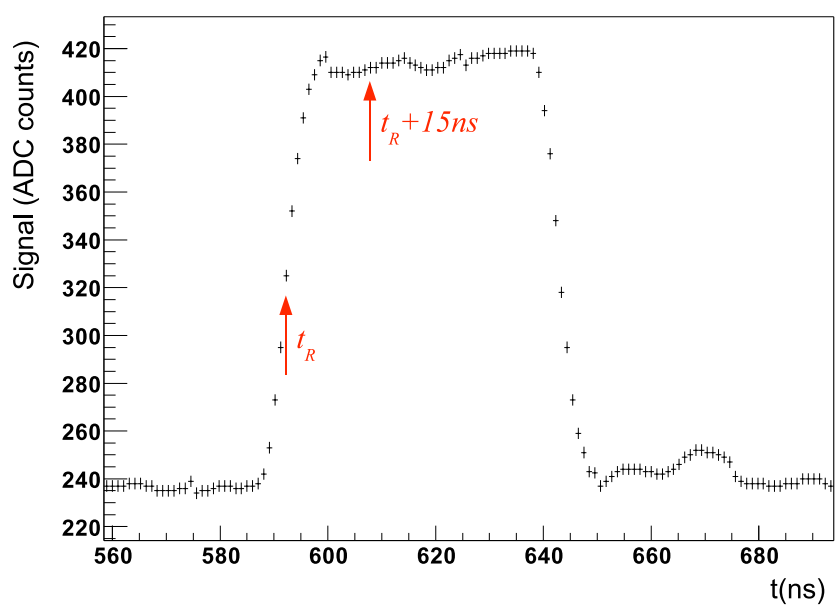

Figure 12. A tick mark sampled during a time alignment. The raising edge and the sampling point are marked. In the picture are reported only those samplings around the tick mark while, during the time alignment, an interval of $1 \mu \mathrm{s}$ is scanned.

dynamic range. This choice avoids having it too near the lower saturation value, while at the same time provides sufficient range for heavily ionizing or multiple minimum ionizing particles (6 MIP equivalent).

These commissiong procedures normally do not need to be repeated often. During the the MTCC, the settings found at the beginning of August were used for the entire data taking period. In this period, external conditions such as temperature and humidity were controlled by the cooling system, which was monitored by DCS/DSS system described in section 2.2.

In order to qualify and monitor detector performance, pedestal runs were taken at least once per day or more if the operating temperature changed by few degrees. Triggers were sent to the modules and all the analogue frames were acquired. For each channel the average and the RMS of the signals were then calculated. These values correspond, respectively, to the pedestal and the raw noise.

As reported previously in this document, if FEDs had to operate in Zero Suppressed mode the pedestal and noise values had to be uploaded into them prior the start of the physics run. The same pedestal and noise values were also transferred to the offline DB for use in the offline reconstruction.

\subsection{Synchronization with external trigger}

A fundamental step in the commissioning of the tracker was the synchronization with the other subdetectors. The signal in each tracker channel is read-out every $25 \mathrm{~ns}$ and stored in a pipeline in the APV. The value is sent to the FED only if a trigger is received. It is therefore necessary to know which pipeline position correspond to a given trigger signal. To this aim the latency, i.e. the time required by the electronic chain to receive the trigger signal and send it to the APV, must be measured. The exact latency value was measured by scanning over an interval of values and searching for the signal coming from cosmic muons.

Unfortunately, the data acquired at SX5 were characterized by an anomalous number of noise clusters due to a few faulty modules (one TOB module and two TIB modules). Moreover, as already pointed out in section 3.4, only in $10^{-4}-10^{-3}$ of the triggered events, depending on the 

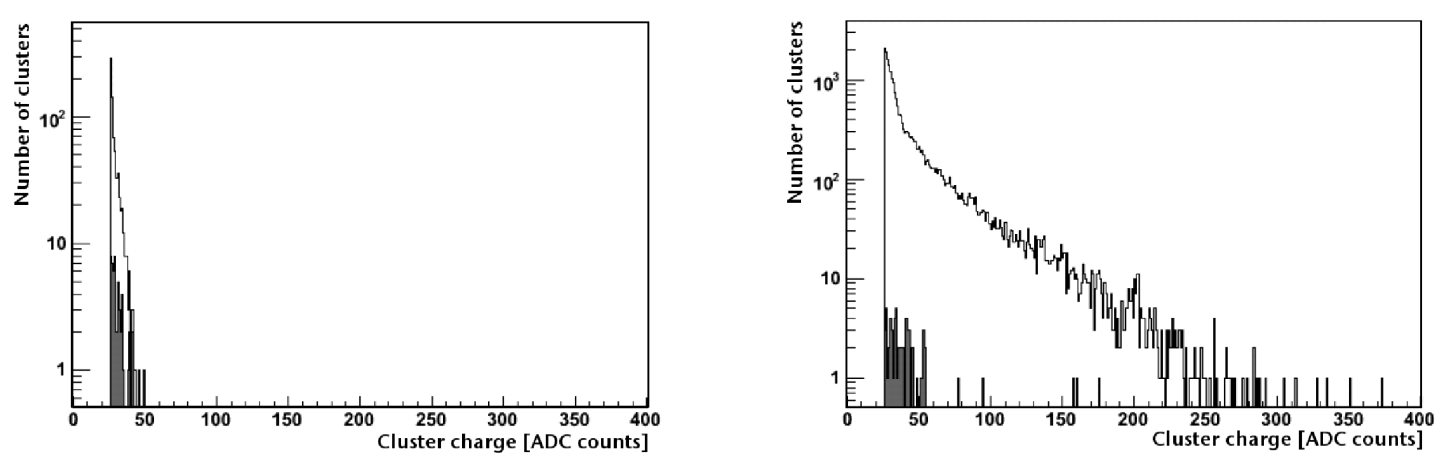

Figure 13. The TIB (left) and TOB (right) cluster charge of each accepted cluster with out-of-time APV latency. The dark-shaded histograms show events with clusters from at least three different layers.
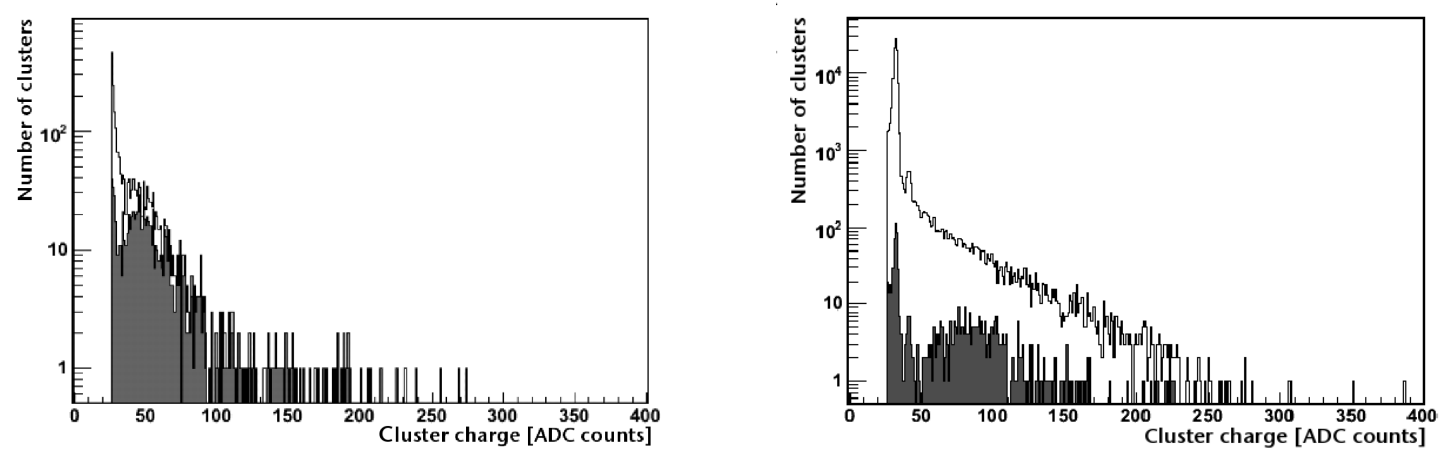

Figure 14. The TIB (left) and TOB (right) cluster charge of each accepted cluster with optimal APV latency. The dark-shaded histograms show events with clusters from at least three different layers.

trigger configuration, the muon was also crossing the tracker. These two combined effects implied that the signal cluster distribution was lost in the tail of the noise cluster distribution, even though the signal-to-noise ratios of the tracker modules were high. In order to extract the signal from this background, the event selection developed for the TIB and TOB (described in section 3.4) was used. This filter led to a dramatic reduction in the number of fake clusters and to the identification of clean signal cluster distributions, even when the latency was not optimal. The cluster charge distribution for TIB and TOB is shown in figures 13 and 14 for two different latency values. The histograms are obtained from the tracker DQM software (section 3.3).

The search for the correct latency value took several weeks as the other subdetectors and the trigger system were also being commissioned during this period. In particular, the latency scan was complicated by a faulty NIM module, which introduced a jitter in the trigger coming from the muon chambers. Therefore, the tracker data from the first few weeks of the MTCC was taken with non-optimal latency values. Eventually, this problem was solved and the correct latency value was determined to be 156 ( $25 \mathrm{~ns}$ units).

This value has been later checked in a more refined offline analysis by looking at the variation of the signal in TIB modules only. The runs used for the analysis are reported in table 8. All these runs were taken in zero-suppression mode with a DT trigger and the magnet switched off. To reject 


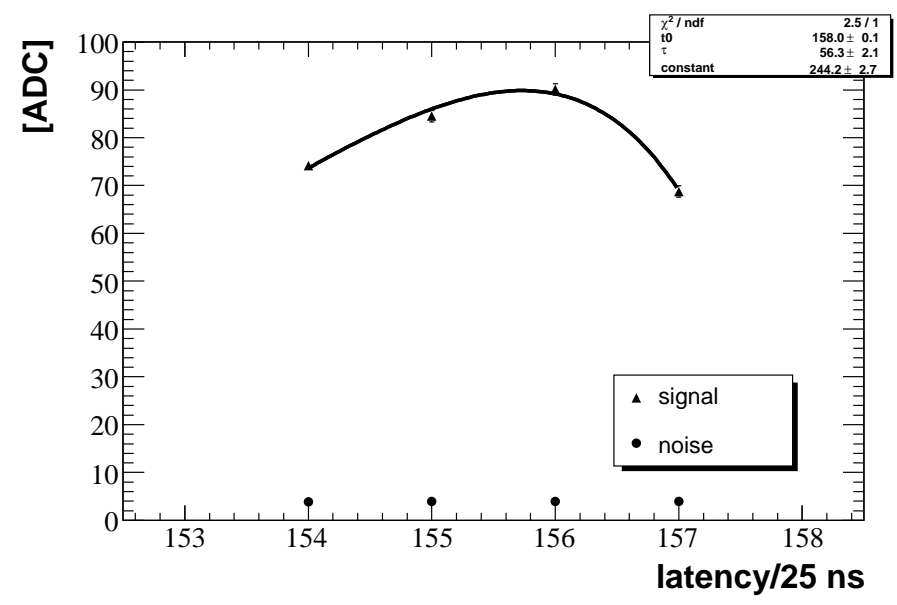

Figure 15. Most probable value from the fit to the cluster charge distribution described in the text for different latency values.

Table 8. Results of the analysis of the latency scan.

\begin{tabular}{|c|c|c|c|}
\hline LAT & N. of Clusters & Noise [ADC] & Signal M.P. [ADC] \\
\hline 155 & 684 & $3.92 \pm 0.02$ & $84.5 \pm 1.2$ \\
154 & 876 & $3.87 \pm 0.02$ & $74.2 \pm 1.0$ \\
156 & 575 & $3.96 \pm 0.02$ & $90.1 \pm 1.2$ \\
157 & 957 & $3.94 \pm 0.02$ & $68.8 \pm 1.2$ \\
\hline
\end{tabular}

tracks at large impact angle, events were selected by requiring clusters both in the TIB and in the TOB but not in the TEC modules. The TIB clusters were required to have $S / N>8$. The distribution of the charge of the cluster collected in the TIB modules was fit with a Landau function convoluted with a Gaussian function. Figure 15 plots the most probable values of the Landau distribution as a function of the latency value. The points obtained were fit with the function

$$
A \frac{t-t_{0}}{\tau} \exp \left(-\frac{t-t_{0}}{\tau}\right)
$$

which describes the CR-RC shaping of the APV in peak mode. After converting the latency units into ns and taking into account that to larger values of latency correspond earlier times, the time constant was found $\tau=56.3 \pm 2.1 \mathrm{~ns}$, in agreement with the APV specification, and the optimal latency value $t_{0}=156.0 \pm 0.1$ (in $25 \mathrm{~ns}$ unit) confirming the value used for the data taking.

\section{Tracker performance}

In this section results of the performance studies are presented. First, the performance of single modules is discussed. Tracking and alignment results are then shown. Based on track information, the study of several quantities like signal-to-noise ratio, response function, $\mathrm{dE} / \mathrm{dx}$ and Lorentz angle is finally presented. 
Table 9. Timing (T) and Pedestal (P) Runs

\begin{tabular}{|l|c|c|c|c|c|c|c|}
\hline Run & 20268 & 20314 & 20373 & 20379 & 20388 & 20391 & 20400 \\
\hline Type & $\mathrm{T}$ & $\mathrm{P}$ & $\mathrm{P}$ & $\mathrm{P}$ & $\mathrm{P}$ & $\mathrm{P}$ & $\mathrm{P}$ \\
\hline Date & $6 / 8$ & $8 / 8$ & $16 / 8$ & $16 / 8$ & $18 / 8$ & $21 / 8$ & $22 / 8$ \\
\hline Field $(\mathrm{T})$ & 0 & 0 & 0 & $>0$ & 0 & 0 & 0 \\
\hline
\end{tabular}

\begin{tabular}{|l|c|c|c|c|c|c|c|}
\hline Run & 20406 & 20410 & 20417 & 20422 & 20424 & 20425 & 20429 \\
\hline Type & $\mathrm{P}$ & $\mathrm{P}$ & $\mathrm{P}$ & $\mathrm{P}$ & $\mathrm{P}$ & $\mathrm{P}$ & $\mathrm{P}$ \\
\hline Date & $23 / 8$ & $24 / 8$ & $26 / 8$ & $26 / 8$ & $27 / 8$ & $28 / 8$ & $29 / 8$ \\
\hline Field (T) & 0 & 0 & 0 & 2.0 & 3.8 & 0 & 0 \\
\hline
\end{tabular}

\subsection{Noise studies and problematic detectors}

Module performance depend on geometry, number of strips hit, readout system and service electronics. Shielding and grounding can in particular affect the detector stability.

Although all modules selected for MTCC were extensively tested at the fabrication facilities during the construction process, they were lower grade with respect to the modules which went to the tracker production line. This fact explains some issues with performance of several modules, observed during data analysis.

Table 9 summarizes the pedestal runs used in the following analyses. They span the full period of cosmic trigger data taking, starting with run 20373 on August 16 and ending with run 20429 on August 29. Typically, a single run, which consists of about two thousand triggers, was taken at the start of each day. Most of the pedestal runs were taken in peak mode, which corresponds to the mode used in the data runs. For the most part, the field was off during these runs. The magnet was ramping during run 20379 and was stable at $2.0 \mathrm{~T}$ and $3.8 \mathrm{~T}$ for runs 20422 and 20424 respectively. These runs allow a comparison of pedestal and noise values to be made for field off and field on conditions.

The time synchronization scan taken on August 3 provide information on the tick mark height and thus the optical gain of each laser mounted on the analog opto-hybrids [36]. A calibration process was applied to the observed noise, which consists in normalizing the tick mark height to 600 ADC counts. This calibration allows a comparison of noise values between different lasers. Figure 16 shows a typical noise profile for a single module with 768 readout channels. Two principal structures can be observed.

1. The 768 strips are separated into six groups with 128 channels each which represent the six APV readout chips. The noise is usually higher at the APV edges.

2. A pair of chips correspond to one laser transferring the signal of 256 strips. Since the gain can be slightly different from laser to laser, the noise exhibits steps.

The laser structure disappears after calibration. 


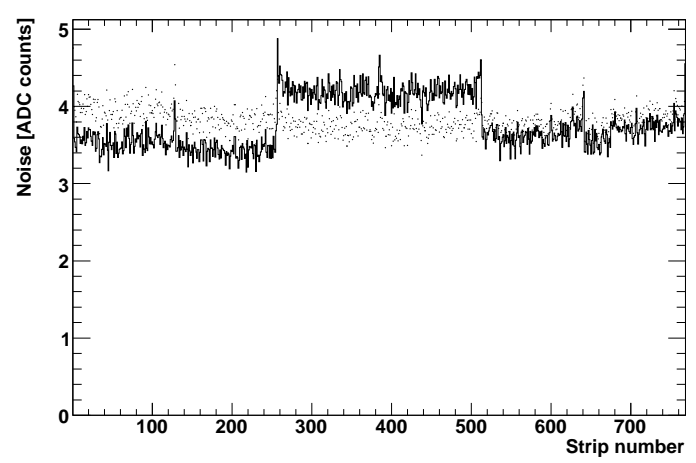

Figure 16. Tick mark height calibrated (dotted) and uncalibrated (solid) noise of module 5.2 on the front petal.

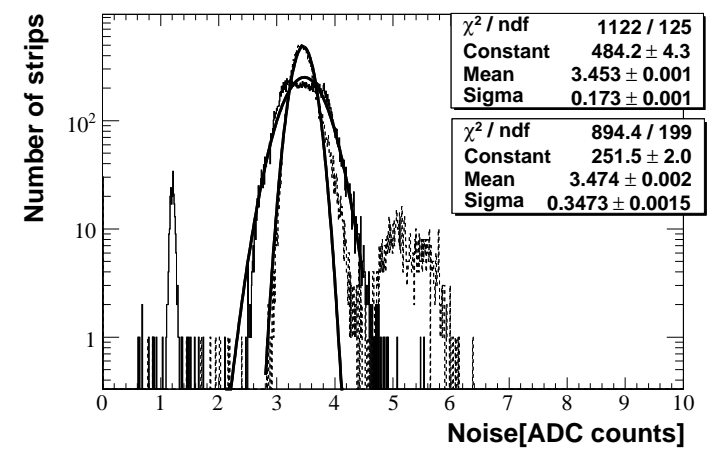

Figure 17. Layer 2 strip noise distribution and Gaussian fit from pedestal run 20314. The noise is shown before (solid histogram) and after (dashed histogram) the tick mark height calibration described in the text.

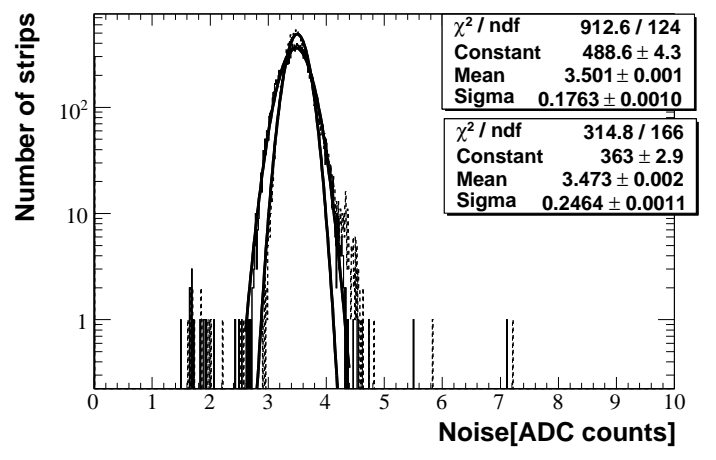

Figure 18. Layer 3 strip noise distribution and Gaussian fit from pedestal run 20314. The noise is shown before (solid histogram) and after (dashed histogram) the tick mark height calibration described in the text.

\subsubsection{TIB modules}

The noise study for the TIB subdetector is summarized in this section. The noise is analyzed separately for layer 2 and layer 3. The pedestal runs from the SX5 data used for this study are 20314, 20388 and 20422.

In figures 17 and 18 the strip noise distribution is shown for layer 2 and layer 3 with and without the tick mark height calibration (run 20314). In both layers, the mean value of the noise was 3.5 ADC counts and the dispersion was reduced after calibration.

The low noise peak near $1 \mathrm{ADC}$ count in layer 2 was due to a single $\mathrm{AOH}$ and disappeared after calibration as shown in figure 19. In one module the noise increased to about 5 ADC counts after calibrations, while it was between three and four ADC counts before (figure 20). The calibration for this module was indeed understood to be wrong because of a fluctuation in the header pulses during the timing run. 


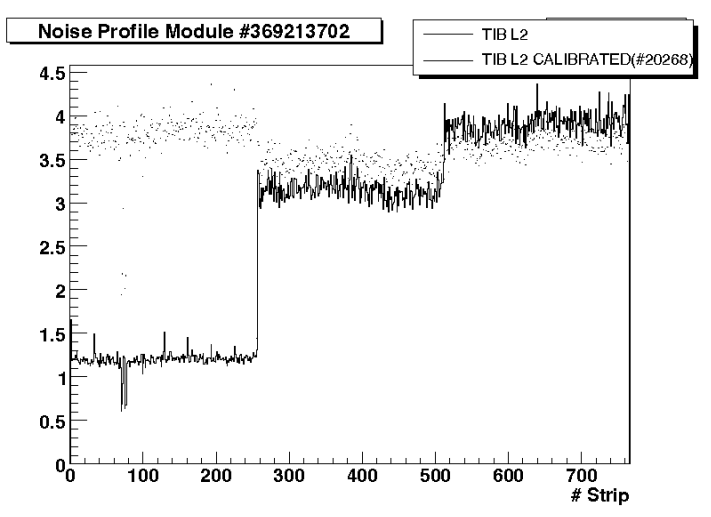

Figure 19. Tick mark height calibrated (dot) and uncalibrated (solid) noise for module 369213702

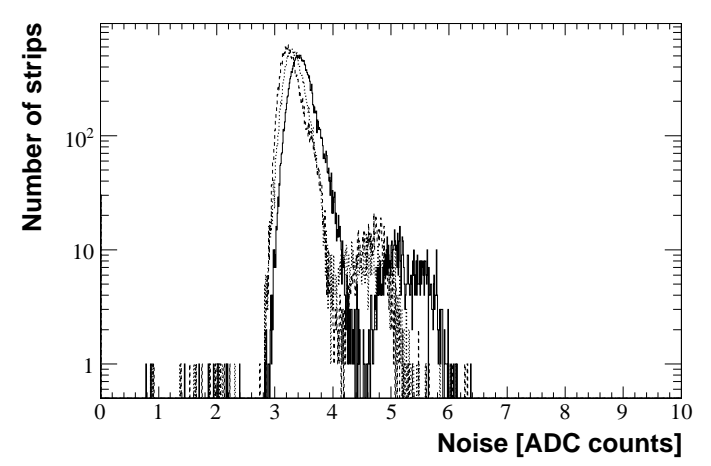

Figure 21. Layer 2 tick mark height calibrated strip noise distribution for three pedestal runs

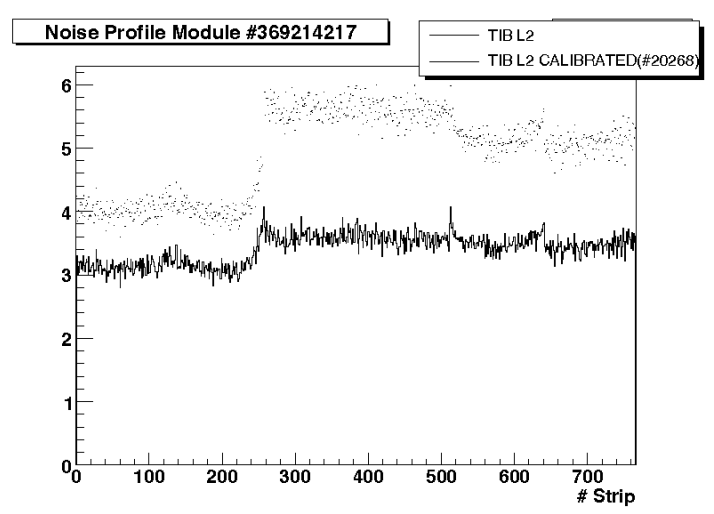

Figure 20. Tick mark height calibrated (dot) and uncalibrated (solid) noise for module 369214217

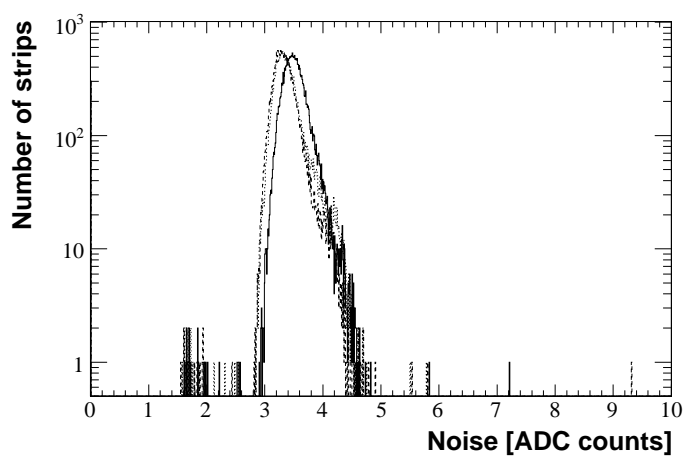

Figure 22. Layer 3 tick mark height calibrated strip noise distribution for three pedestal runs

The TIB layer 3 behavior was even more uniform than layer 2. Only very few strips had low noise values, both before and after calibration.

The strip noise distributions for three different runs, 20314, 20388 and 20422, are shown in figures 21 and 22. These three runs span a period of about one month and the latter was taken with a magnetic field value of $2 \mathrm{~T}$. The stability of the system over such a long period and conditions is remarkably good.

As a further cross-check, the noise in runs 20388 and 20422 has been compared strip-by-strip with run 20314. The difference is plotted in figures 23 and 24. For both layer 2 and 3 the average value of the distribution is negative, which means the noise reduced with time. This can be due to a small difference in the temperature, which in turn could have been determined by a change in the cooling flux. However, the absolute difference in ADC counts is low and compatible with temperature fluctuations. These results confirm that the strip noise was stable for the full data taking period. 


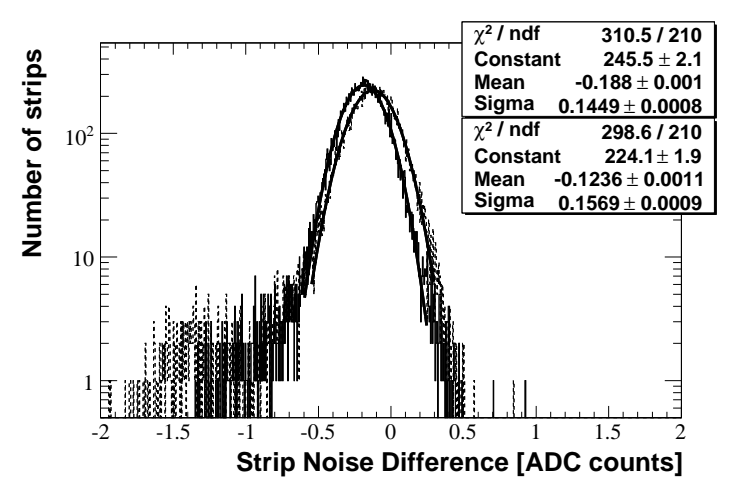

Figure 23. Tick mark height calibrated noise strip difference distribution for layer 2

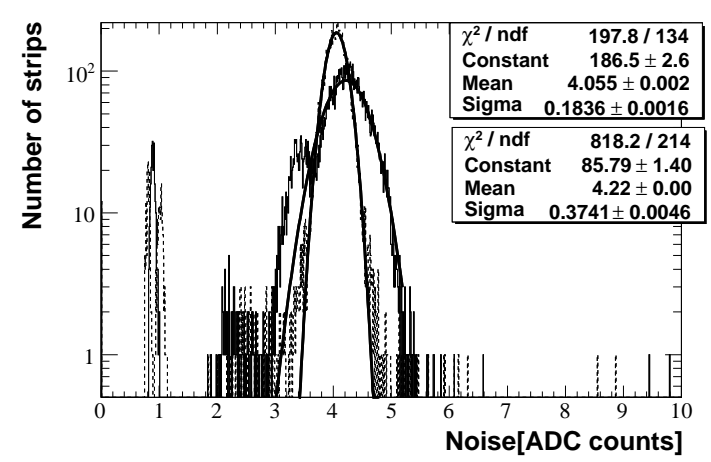

Figure 25. Layer 1 strip noise distribution and gaussian fit from pedestal run 20314. The noise is shown before (solid histogram) and after (dashed histogram) the tick mark height calibration described in the text.

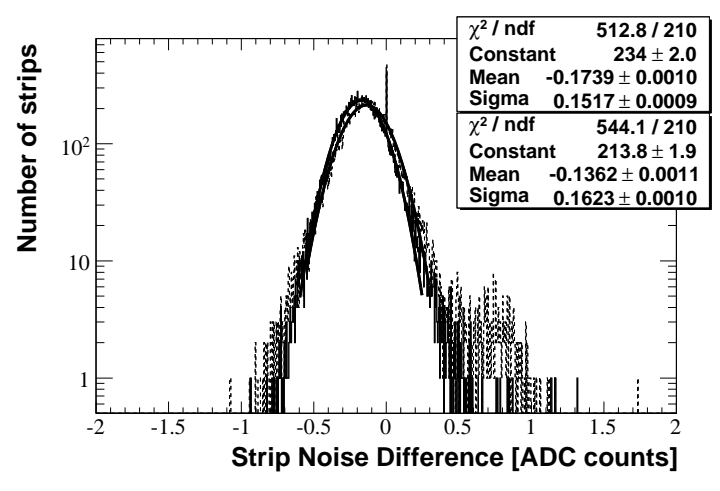

Figure 24. Tick mark height calibrated noise strip difference distribution for layer 3

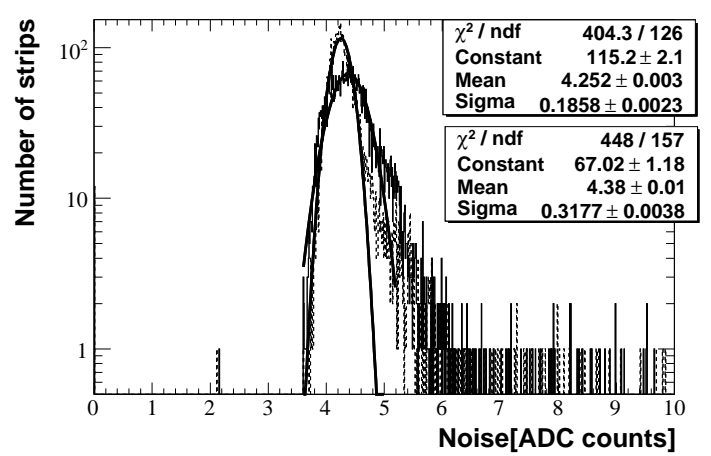

Figure 26. Layer 5 strip noise distribution and gaussian fit from pedestal run 20314. The noise is shown before (solid histogram) and after (dashed histogram) the tick mark height calibration described in the text.

\subsubsection{TOB modules}

All the TOB modules used in the MTCC tracker were older, pre-production versions: both hybrids and sensors came from non-qualified batches and lots. These modules also contained a higher percentage of open channels than the modules used to construct the CMS TOB, and one of the selected modules had a history of APV problems.

Figures $25 \sqrt{30}$ show the summary distributions for the noise in layer 1 and 5 of the TOB. Runs 20314, 20388 and 20422 were used, as it was done for the TIB in the previous section.

One module exhibited problematic pedestal and noise values as early as pedestal run 20314 and, by run 20379 , the majority of the channels had saturated pedestals. This feature is visible as 


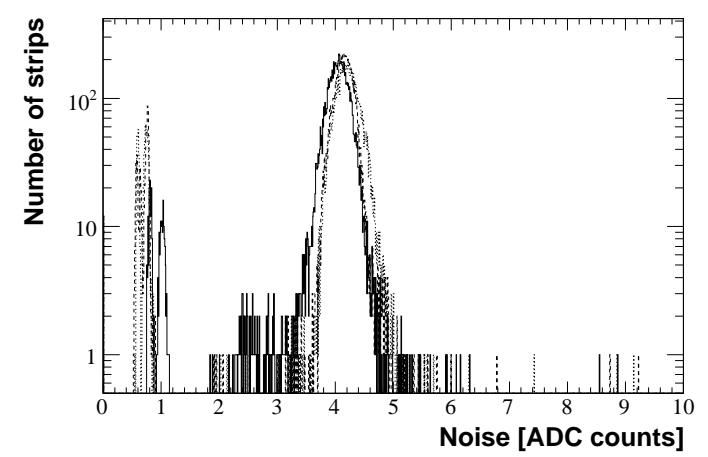

Figure 27. TOB Layer 1 tick mark height calibrated strip noise distribution for three pedestal runs

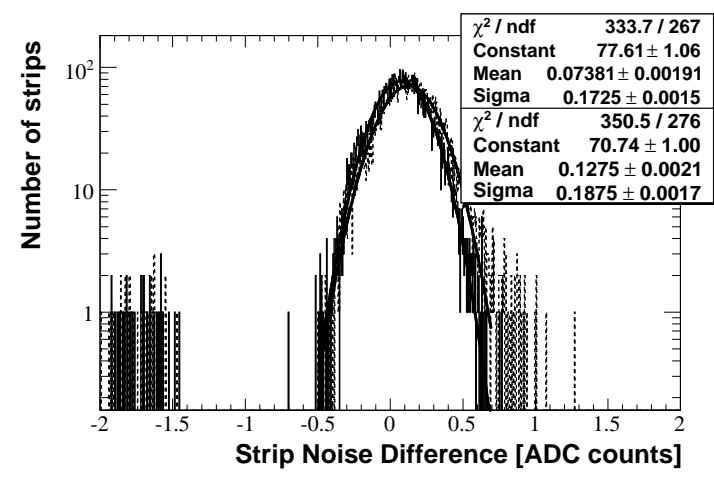

Figure 29. Tick mark height calibrated noise strip difference distribution for layer 1

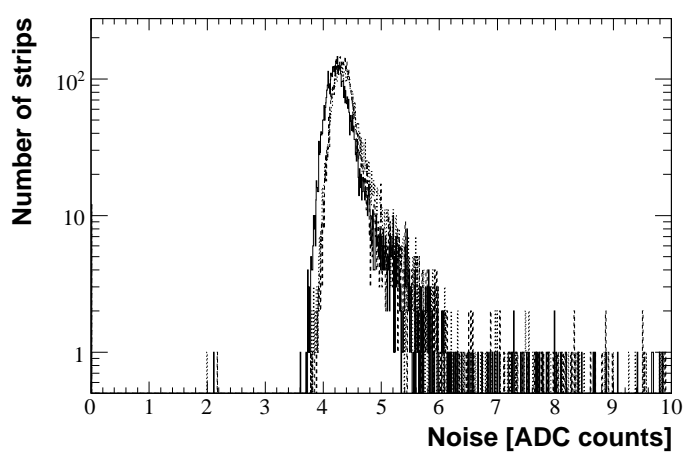

Figure 28. TOB Layer 5 tick mark height calibrated strip noise distribution for three pedestal runs

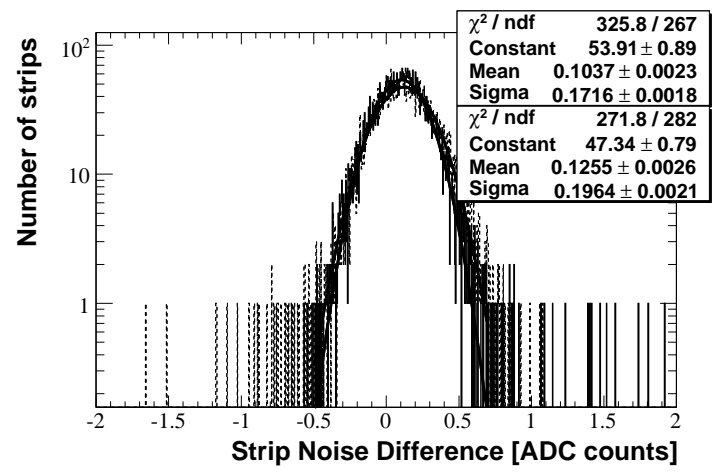

Figure 30. Tick mark height calibrated noise strip difference distribution for layer 5

a low noise peak at $1 \mathrm{ADC}$ count in figures 25 and 27 . The saturated pedestal distribution can be seen in figure 31. The condition of saturated pedestals remained unchanged throughout the MTCC run. This module was known to have problems from earlier testing at CERN and was included in the MTCC tracker only in the absence of other options.

Two of the APVs on one module developed a problem at some point between runs 20391 and 20400. Figure 32 shows the change in pedestal values for APVs 5 and 6 in this module. The solid line shows the initial values and the dashed line the final values. This condition of high pedestals for the last two APV's persisted throughout the MTCC run. Unlike module 71972, there was no hint of problems for this module previous testing at CERN. Despite this change in pedestal value, the noise for this module was practically unchanged.

In general increased noise was observed at the boundaries between APVs on modules. This 


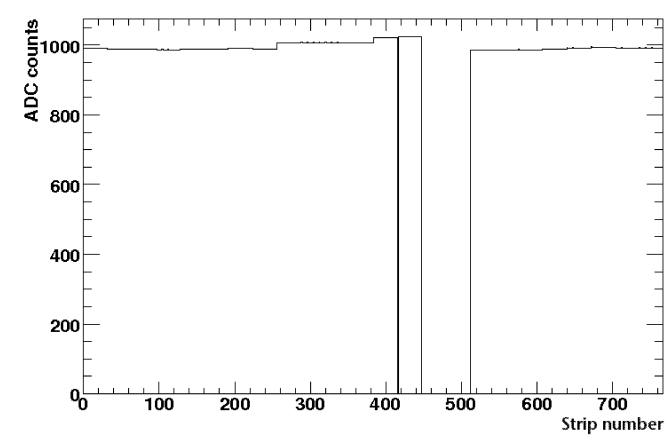

Figure 31. Saturated pedestal module.

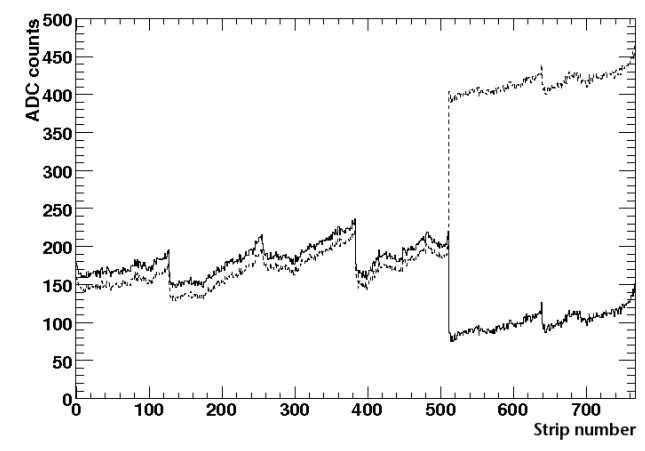

Figure 32. Module with high pedestals on last two APVs. Pedestals are shown for run 20391 (solid) and run 20400 (dashed).

effect was noted even during single module tests. However, rod testing, both at the production sites and at the integration site at CERN, showed a broader increase of noise at the APV edges. This peculiar noise distribution, hereafter called wing effect, is due to the formation of an eventby-event-different slope across the 128 APV channels: as this fluctuation is not flat, the common mode subtraction doesn't remove it at the edges.

Module positions within a rod are labeled in increasing $z$ order, so that the sixth position is near the readout end of the rod. The source of noise leading to the wing effect appears to be coupled most strongly to the position six modules and diminishes with decreasing position number. The noise is also most strongly coupled to the outer APVs of the end modules.

Figure 33 shows the noise data for modules 1 and 6 of the first rod in the first layer of the MTCC TOB system. A similar effect can be seen in the second rod of the first TOB layer and both of the (SS6) rods in the second layer barely show this effect. This difference-between SS4 and SS6 rods-has been noted in TOB integration testing. Double-sided rods, which contain 6 pairs of 4 APV modules, exhibit a wing effect that is intermediate in magnitude between the SS6 and SS4 rods.

A grounding and shielding schema which greatly reduce the wing effect has been developed in the final tracker assembly. In addition it may be possible to reduce further the wing effect by adding a linear term (slope across the 128 channels of an APV) to the common mode noise subtraction algorithm in the FED. This technique has been shown to be effective in an event-by-event study of post-FED raw pedestal data.

A comparison of the noise at the MTCC was made with data from the TOB module construction sites. All the modules have been tested using the APV Readout Controller (ARC) system [34] before they were mounted on the rod structures. Therefore the analog opto-hybrid was not yet included in the read-out chain. To aid the comparison, the signal amplitudes measured with the ARC system have been increased by a factor of three, which corresponds roughly to the additional gain due to the lasers on the AOH. Furthermore, in the ARC data the common-mode noise subtraction was not performed. An open channel, i.e. a channel disconnected because of a missing bond or 


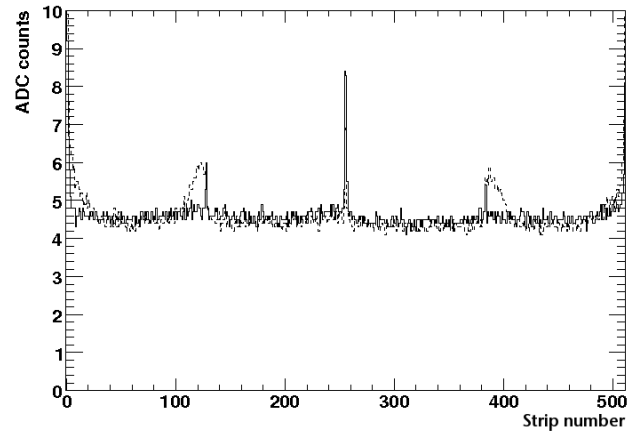

Figure 33. Noise on wings of the outer APV's near rod CCUM for module 1 (solid) and module 6 (dashed).

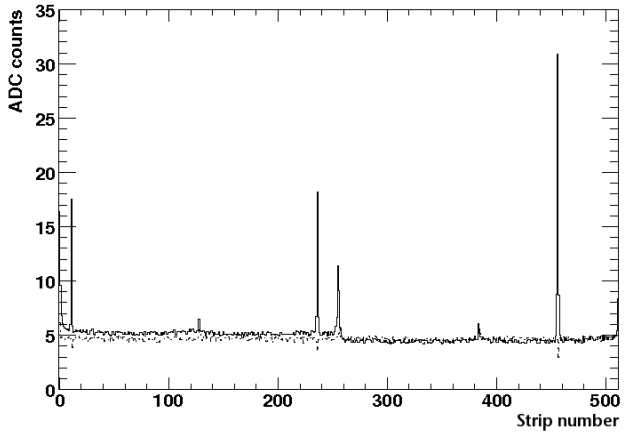

Figure 34. Comparison of MTCC data (solid) with construction data (dotted).

with a break in the metallization of the strip, has a lower noise because of the reduced capacitive load on the APV input. Most of the observed common mode noise couples in through the sensor and open channels are therefore largerly unaffected. As a result, when common mode noise subtraction is made for all 128 APV channels, the common mode noise is effectively reflected in open channels. This effect has been observed in all four APV modules, while it is absent for six APV ones, which have a lower common-mode noise. Figure 34 shows a comparison of ARC and MTCC data for module 6184, a 4 APV module, where this effect is evident for the opens at channels 12 , 237 and 457.

Aside from the module with pre-existing problems and the module with the two high pedestal APV's, the TOB pedestals and noise were stable. There is some indication from the electronic $\log$ that a significant shift in pedestal values occurred early on in the run. Figure 35 shows a comparisons of pedestals for module 6180 from three pedestal runs. From the figure it is clear that a 15\% upward shift in values occurred at some point after run 20401. However, the same figure shows essentially no difference in pedestal values between latter runs 20424, where the field was at $3.8 \mathrm{~T}$, and run 20429, where the field was off. Even where the pedestals did change there was little change in noise, as is illustrated in figure 36 for the same module and the same pedestal runs.

Typically, the noise values were between 4 and 5 ADC counts. This value is consistent with the experience from TOB integration for peak mode. Discounting the two problem modules noted above, bad channels were also stable throughout the run.

\subsubsection{TEC modules}

To study the noise behaviour of the 34 TEC modules available in the MTCC, the same procedure is performed as for TIB and TOB modules. In figures 38 to 41 the calibrated and uncalibrated values of noise in run 20314 are shown. Since the noise increases with increasing strip length from ring 4 to ring 7 , the distributions are shown individually for each ring. 


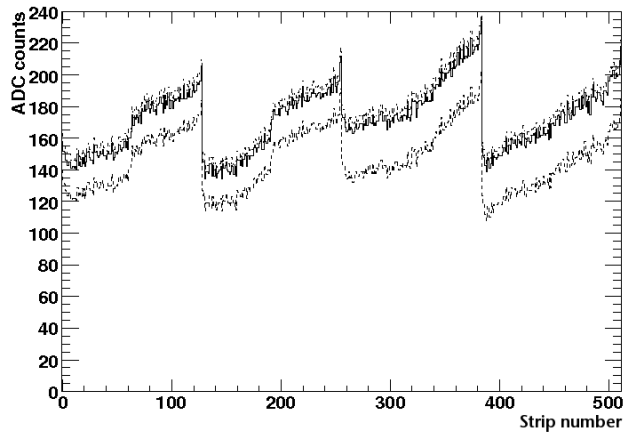

Figure 35. Module 6180 pedestals in run 20401 (dashed), run 20424 (solid) and run 20429 (dotted).

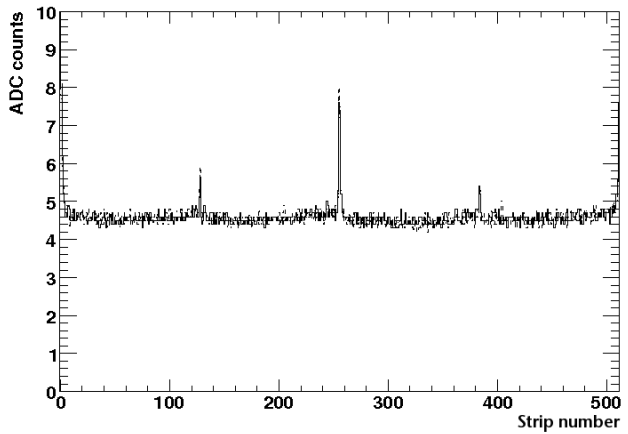

Figure 36. Module 6180 noise in run 20401 (dashed), run 20424 (solid), and run 20429 (dotted).

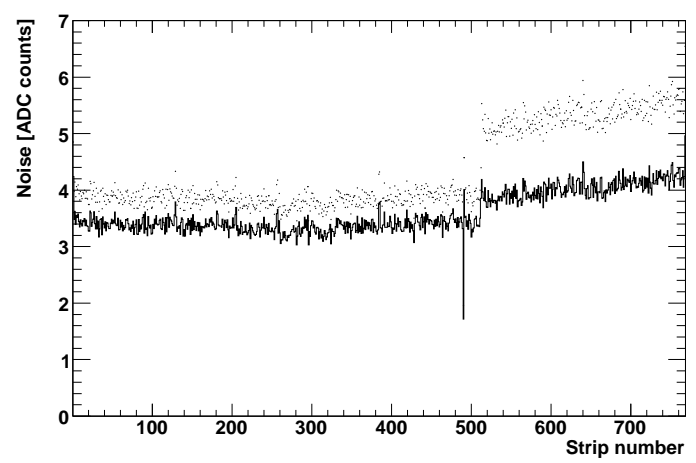

Figure 37. Tick mark height calibrated (dot) and uncalibrated (solid) noise of TEC ring 5 module with high noise in one APV pair.

In general, the width of the noise distributions is reduced after applying the calibration due to the adjustment of the laser gains. Only for ring 5 modules does the calibration lead to a second peak at higher noise values. This behaviour originates from a single module, which is mounted on position 5.3 on the front petal. This effect is evidenced in figure 37 where the noise increases for the third laser (channels 513-768) following calibration. The increase is attributed to fluctuations of the tick mark for this laser during the timing run, as already observed for one TIB module.

To verify the stability of the noise behaviour pedestal runs 20314, 20388 and 20422, taken during a period of approximately one month, were compared with each other. Figures 42 to 45 show the noise distributions obtained from the three runs. The variation with time is found to be very small.

To verify this behaviour on a single strip basis, the distribution of the noise difference for each individual strip is shown in figures 46 to 49 with respect to run 20314. Dashed lines correspond 

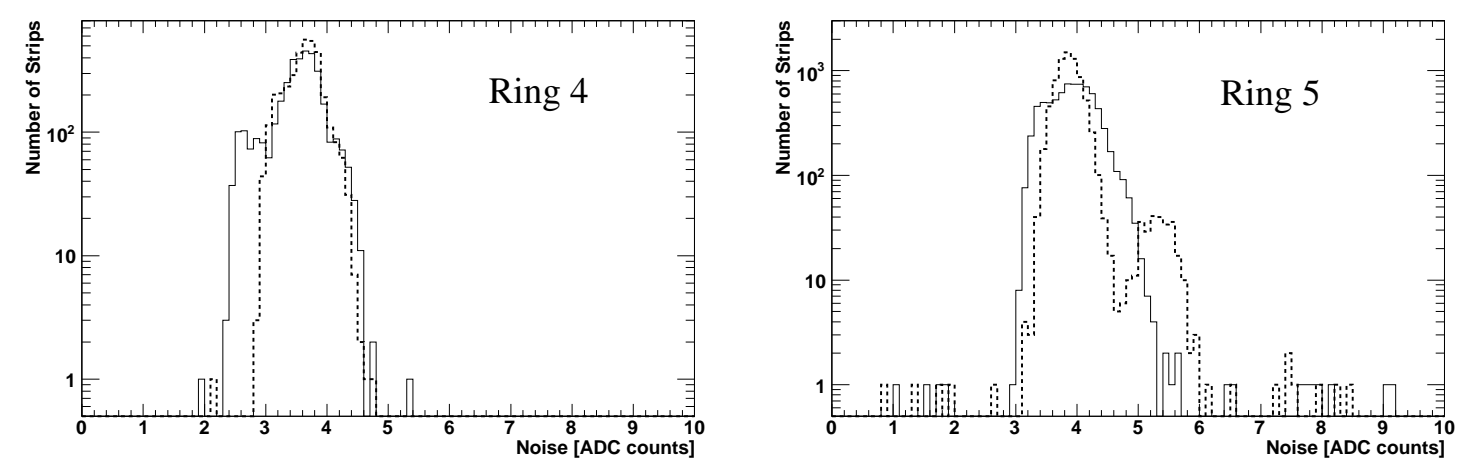

Figure 38. Tick mark height calibrated (dotted) and Figure 39. Tick mark height calibrated (dotted) and uncalibrated (solid) noise of ring 4 modules. uncalibrated (solid) noise of ring 5 modules.
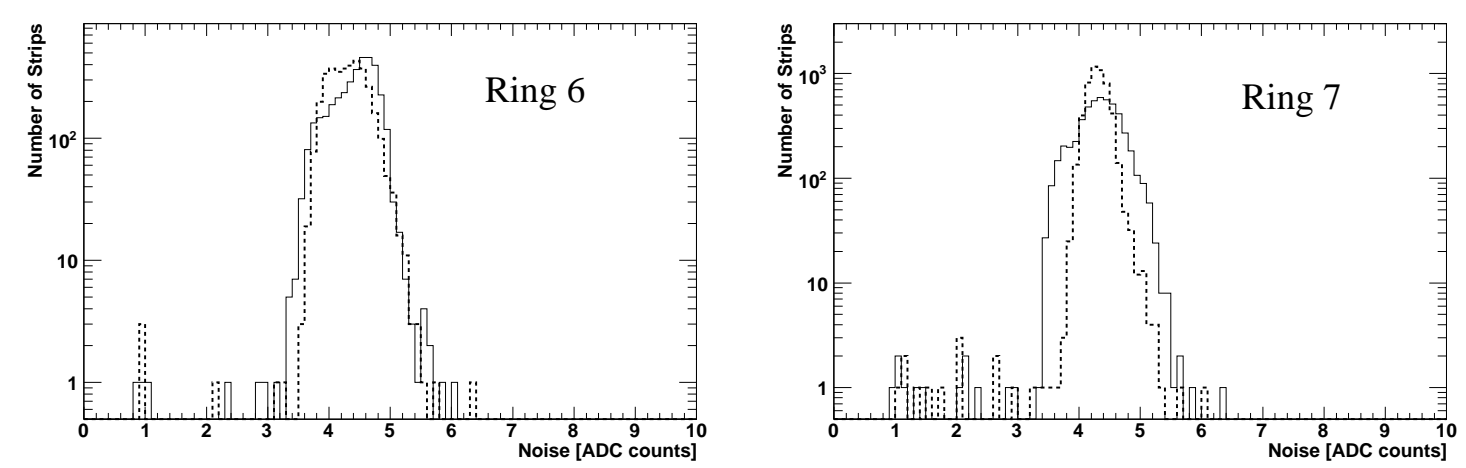

Figure 40. Tick mark height calibrated (dotted) and Figure 41. Tick mark height calibrated (dotted) and uncalibrated (solid) noise of ring 6 modules. uncalibrated (solid) noise of ring 7 modules.
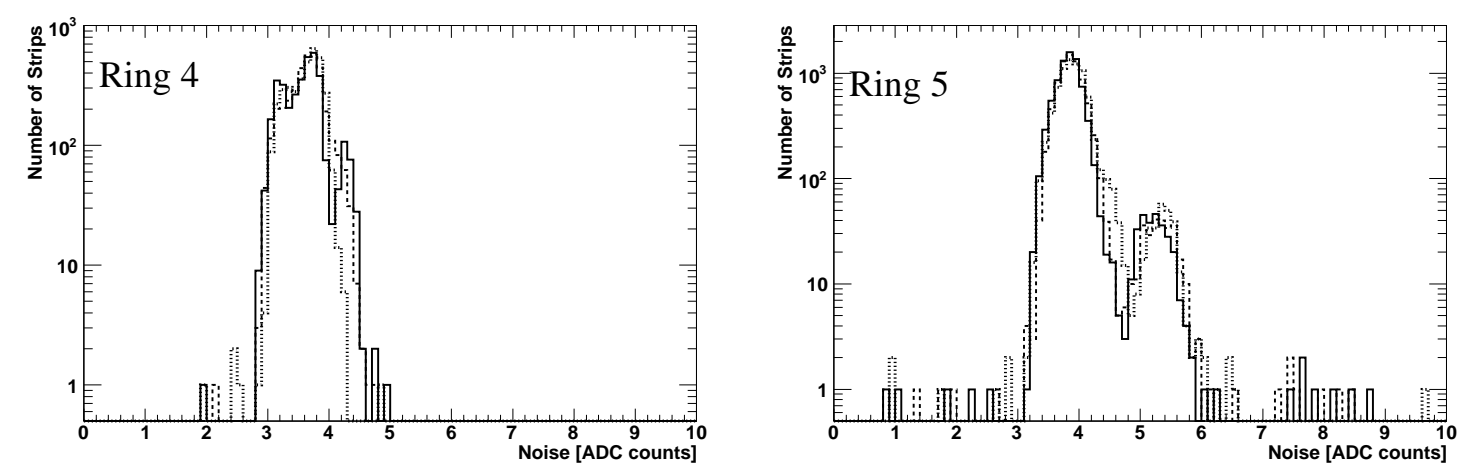

Figure 42. Tick mark height calibrated noise of ring Figure 43. Tick mark height calibrated noise of ring 4 modules for three pedestal runs. 5 modules for three pedestal runs.

to run 20388 and solid lines to run 20422. All differences are in agreement with zero and all distributions are well described by a Gaussian distribution. Only the comparison between the second run under investigation and the reference run for ring 5 modules shows a significant number 

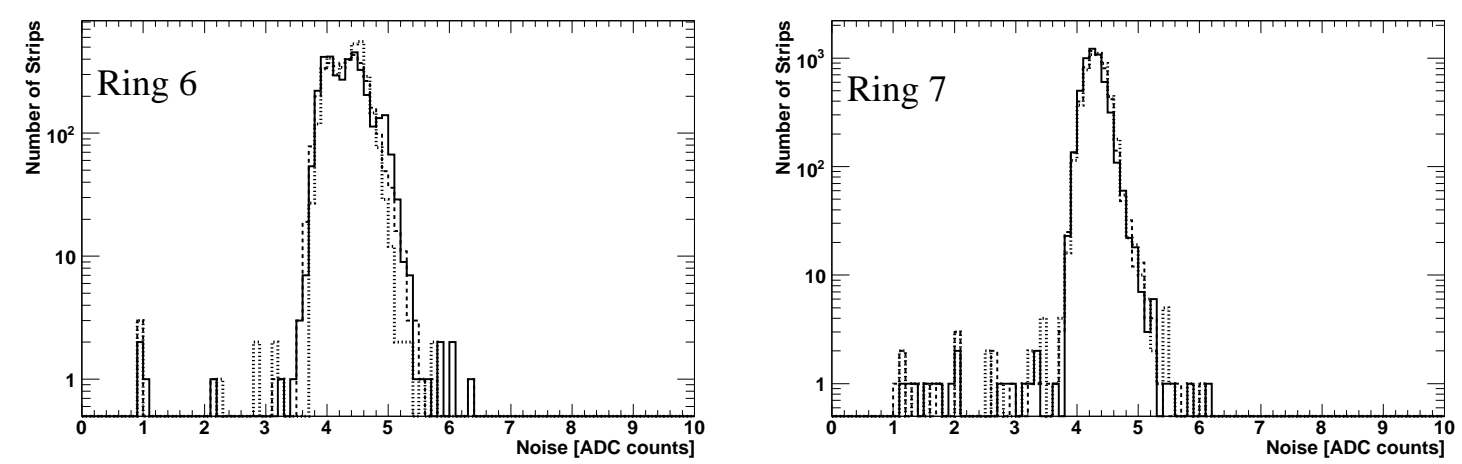

Figure 44. Tick mark height calibrated noise of ring Figure 45. Tick mark height calibrated noise of ring 6 modules for three pedestal runs. 7 modules for three pedestal runs.
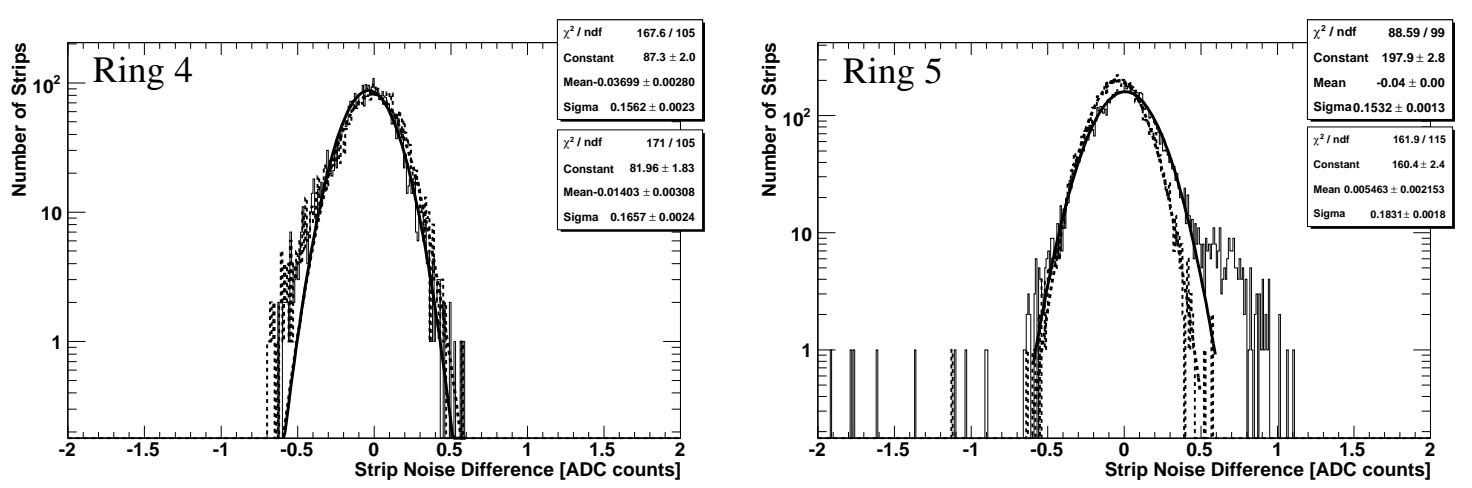

Figure 46. Tick mark height calibrated noise differ- Figure 47. Tick mark height calibrated noise difference of ring 4 modules. ence of ring 5 modules.
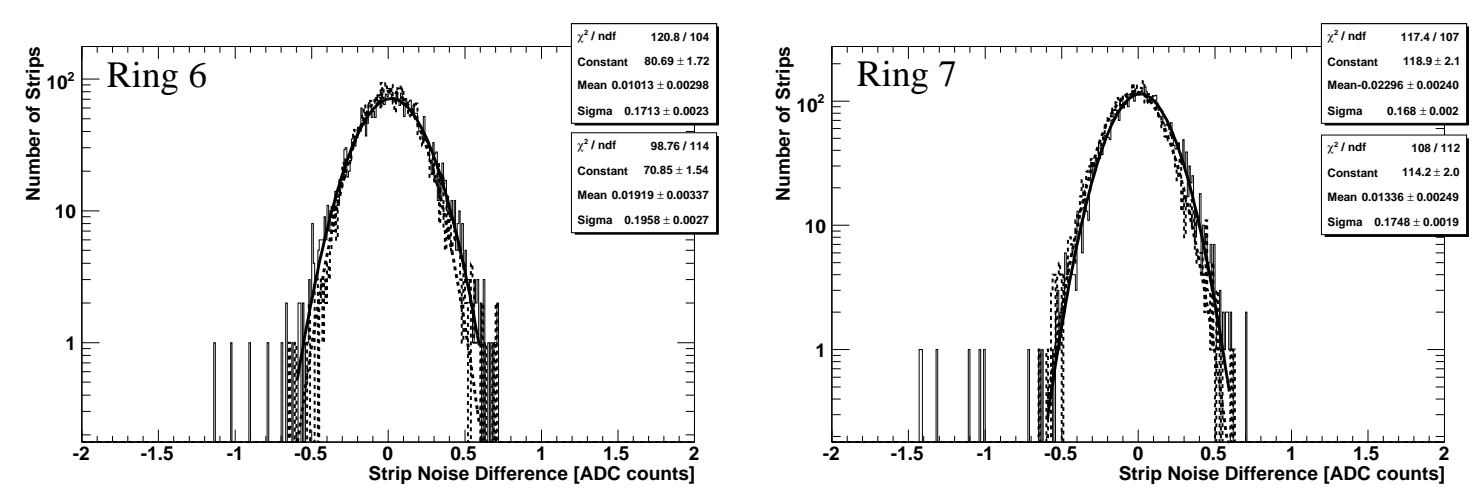

Figure 48. Tick mark height calibrated noise differ- Figure 49. Tick mark height calibrated noise difference of ring 6 modules. ence of ring 7 modules.

of strips with a higher difference in noise than the other channels. This behaviour is caused by a group of 256 strips of a single module as shown in figure 50. This silicon sensor on position 5.3 on the front petal has increased noise at the second laser during the last pedestal run analysed here. 

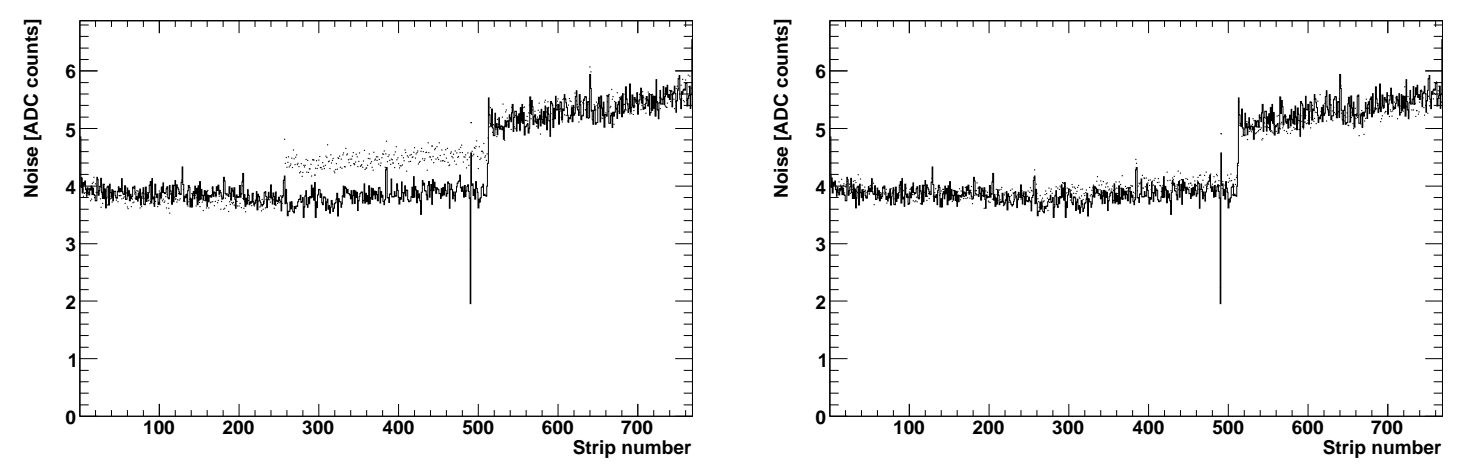

Figure 50. Tick mark height calibrated noise of mod- Figure 51. Tick mark height calibrated noise of module 5.3 for the second pedestal run under investigation ule 5.3 for the first pedestal run under investigation (dotted) and the reference run (solid).

(dotted) and the reference run (solid).

This increase can be induced by a temporary change in the gain conditions, for example, due to a difference in the operating temperature. The deviation does not appear in the first pedestal run under investigation (figure 51).

Based on these results it can be concluded that the noise of the TEC modules was stable during the entire data taking period.

\subsection{Tracking and alignment}

Three different alignment studies were performed on the MTCC tracker setup and are presented here. A first set of global corrections was determined using cosmics collected before moving the tracker to SX5, in order to provide fast feedback to other analyses depending on track reconstruction. A detailed study of module-by-module alignment was done on the same data to estimate the best reachable resolution with cosmic data. Finally, data collected at SX5 was analysed with the HIP algorithm (section 3.1.3).

\subsubsection{TIB survey measurements}

A set of survey measurements was made on the MTCC TIB structures. These measurements were used to determine the centre points and orientations of the sensors, which in turn served as the starting point for the alignment studies.

Prior to mounting modules, measurements on the TIB layer structures had been made using a DEA measurement machine [35] with a touch-trigger probe capable of measuring the coordinates of up to 2000 points in three hours with an accuracy better than $50 \mu \mathrm{m}$. A cylindrical reference frame, similar to that which will be used for CMS, was defined for each TIB layer by measuring the coordinates of the surface of the bushings located at the layer flanges. For each module location a set of measurements and operations was done in order to determine the orientation of the ledge plane and define a local right-handed reference frame, based on CMSSW.

From the DEA measurements, the engineering drawings of the module frame, and the Gantry measurements, the centre and orientation of the silicon active areas were computed and compared with the expected values. Table 10 summarizes the measured and expected values for the polar 
Table 10. Measured value and distribution spread of the the MTCC TIB layer cylindre polar radius compared with the design value. The polar radius is measured in the $x y$ plane from the $z$ axis to the $r \phi$ sensor center.

\begin{tabular}{|c|c|c|c|c|}
\hline \multicolumn{2}{|c|}{ MTCC TIB } & \multicolumn{3}{|c|}{ Polar Radius [cm] } \\
\hline Layer & Part & Mean & RMS & Design \\
\hline 2 & int & 31.96 & 0.03 & 32.19 \\
\hline 2 & ext & 35.41 & 0.07 & 35.61 \\
\hline 3 & int & 40.35 & 0.01 & 40.25 \\
\hline 3 & ext & 43.52 & 0.02 & 43.45 \\
\hline
\end{tabular}

Table 11. Residuals between the measured ( $\left.z^{\text {meas }}\right)$ and expected $\left(z^{\text {proj }}\right)$ position of sensor centres along the $z$ direction for the internal and external surfaces of the MTCC TIB layers.

\begin{tabular}{|c|c|c|c|}
\hline \multicolumn{2}{|c|}{ MTCC TIB } & \multicolumn{2}{c|}{$z^{\text {meas }}-z^{\text {proj }}[\mu \mathrm{m}]$} \\
\hline Layer & Part & Mean & \multicolumn{1}{c|}{ RMS } \\
\hline 2 & int & -476 & 74 \\
\hline 2 & ext & -9 & 141 \\
\hline 3 & int & +126 & 57 \\
\hline 3 & ext & -199 & 62 \\
\hline
\end{tabular}

Table 12. Measured value and distribution spread of the the MTCC TIB ledge plane tilt angle compared with the design value.

\begin{tabular}{|c|c|c|c|c|}
\hline \multicolumn{2}{|c|}{ TIB+ } & \multicolumn{3}{c|}{ Tilt Angle [deg] } \\
\hline Layer & Part & Mean & RMS & Design \\
\hline 2 & int & 8.72 & 0.19 & 9 \\
\hline 2 & ext & 10.36 & 0.17 & 9 \\
\hline 3 & int & 8.79 & 0.18 & 9 \\
\hline 3 & ext & 8.74 & 0.27 & 9 \\
\hline
\end{tabular}

radius for each MTCC TIB sub-layer. Modules mounted on the external or the internal part of the mechanical structure are shown separately. The polar radius is defined as the radial distance from the $z$ axis to the sensor centre.

The mean and RMS of the distributions of the residuals between the measured and expected values of the $z$ coordinate are shown in table 11. The large discrepancy in $z$ for the MTCC TIB Layer 2 is due to the structure used, which is not a semi-cylindre made of carbon fibre as is the case with the actual CMS TIB, but only a smaller structure made out of PVC.

The tilt angle is defined as the angle between the normal to the sensor plane and the radial direction in $x y$ plane. Measurements of the angles, summarized in table 12 , are consistent with the design value of 9 degrees.

The measured coordinates of the sensor active area centers and local reference frames are included in the TIB construction database and used to align the MTCC TIB modules within each layer (see section 5.2). 
Table 13. $\Delta \phi$ estimate from a Gaussian fit to the difference in slope between TIB and TOB segments.

\begin{tabular}{|c|c|}
\hline$<\Delta \phi>(\mathrm{rad})$ & $0.0602 \pm 0.0004$ \\
\hline$\sigma_{\Delta \phi}(\mathrm{rad})$ & $0.0092 \pm 0.0003$ \\
\hline
\end{tabular}

Table 14. Translation of TIB with respect to TOB.

\begin{tabular}{|c|c|}
\hline$\Delta x(\mathrm{~cm})$ & $-4.188 \pm 0.012$ \\
\hline$\Delta y(\mathrm{~cm})$ & $0.082 \pm 0.014$ \\
\hline
\end{tabular}
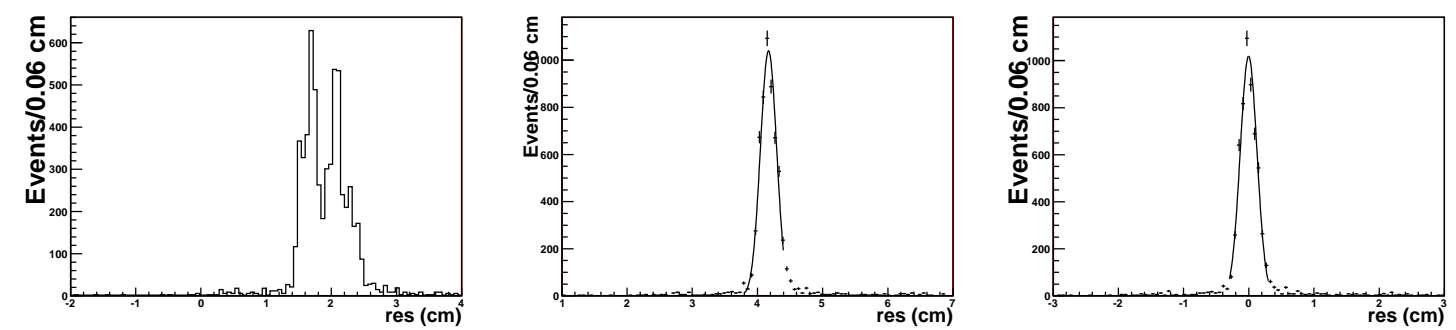

Figure 52. TIB residual before any correction (left), after rotation (centre), after rotation and translations (right)

\subsubsection{Pre-alignment in the tracker assembly hall}

This alignment study was performed with data collected using scintillators in the tracker assembly hall (section 2.1). A standalone algorithm which uses only the information on the position of hits in the $(x, y)$ plane was applied to TIB and TOB.The algorithm calculates corrections of the relative position of TIB with respect to TOB in this plane. The corrections are parameterized by two translations and a rotation: a shift $\Delta x$ along the $x$ axis, a shift $\Delta y$ along the $y$ axis and a rotation $\Delta \phi$ around the $z$ axis.

The algorithm relies on a linear fit of straight tracks: this data were collected with the magnetic field off. Only events with hits in all four layers were considered. The selected sample represents 3155 events out of the original sample of 12340 events.

The rotation of TIB with respect to TOB was first determined to correct a double-peak structure in the TIB residuals (figure 52, left plot). The distribution of the angle between track segments in TIB and track segments in TOB was interpolated by a Gaussian distribution, the mean of which gives an estimate of $\Delta \phi$ (table 13). After correction, the two peaks merge, as shown in figure 52.

$\Delta x$ and $\Delta y$ are consecutively obtained by the minimization of $\chi^{2}$ variables constructed from the mean residual and errors, determined from a Gaussian fit to the residual distribution. The results of the minimization are summarized in table 14. The large shift in the $x$ coordinate reflects the low precision on the relative position of TIB and TOB substructures in the MTCC setup, which has no relation with the ultimate precision on the final tracker assembly.

The residual distribution after correction is centred at zero, as shown in the figure 52 . After applying these corrections, the number of reconstructed tracks increased from 952 to 2526 and the spatial resolution improved as well. 

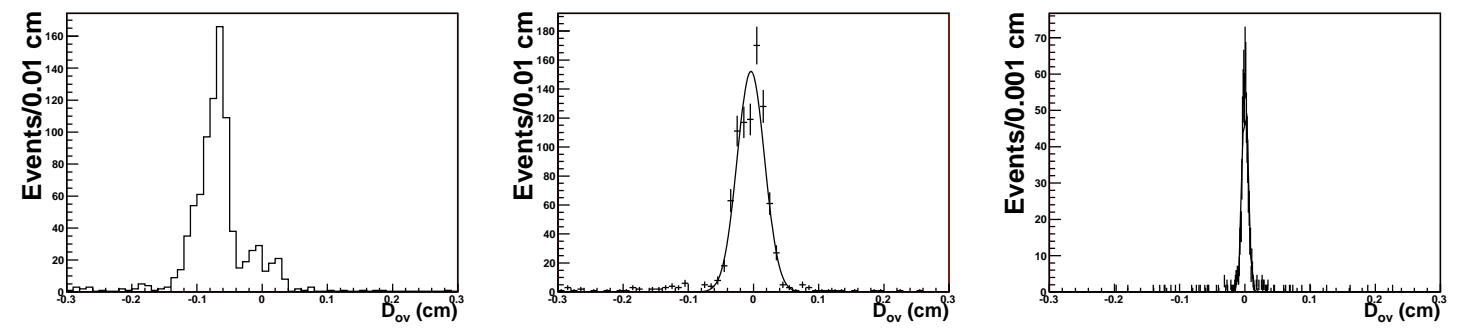

Figure 53. $\Delta_{\mathrm{OV}}$ distribution after the global pre-alignment (left), after moving the external TIB L3 (centre), after correcting locally (right).

\subsubsection{Local alignment sensitivity with assembly data}

Another alignment analysis was performed with data collected in the tracker assembly hall, using scintillators to trigger cosmic muons, in order to assess the ultimate sensitivity of cosmic data on the position of individual modules. The study was done in the overlap regions of the TIB, where modules in different layers cover the same $z$ region. The small lever arm between these modules ensure excellent resolution and thus good sensitivity to sensor positions.

Events with one hit in TIB L2 and two hits in TIB L3 were selected after applying the prealignment corrections. A track segment is constructed from the two outermost hits. The alignment estimator, $\Delta_{\mathrm{OV}}$, is defined as the $x$ residual between this segment and the innermost hit of TIB L3. The distribution of $\Delta_{\mathrm{OV}}$ before correction (figure 53, left plot) is not centered at zero, indicating a displacement of the internal side of TIB L3 with respect to the external side. By minimizing the sum of the residuals squared, we obtain a shift of $1.2 \mathrm{~mm}$.

The residuals were found to depend linearly on the track angle. The individual module positions were then corrected in $x$ and $y$ to compensate for this effect. The final distribution of the residuals is shown in figure 53 (right plot). The modules in TIB L3 have $120 \mu \mathrm{m}$ pitch, hence the expected resolution is $120 \mu \mathrm{m} / \sqrt{12}=35 \mu \mathrm{m}$ for orthogonally incident tracks, crossing one strip alone, and slightly better for tracks crossing two or three strips. From the width of this distribution, which is about $45 \mu \mathrm{m}$, and correcting for the geometrical factor from extrapolation, the estimated point resolution, which includes all the residual effects from mis-alignment, multiple scattering, and intrinsic resolution, is about $30 \mu \mathrm{m}$, which is consistent with the expected value.

\subsubsection{Alignment analysis of MTCC data}

As previously mentioned (section 3.1.3), the final alignment corrections were obtained with MTCC data from the HIP algorithm. This alignment analysis was done on the largest sample of events with the magnetic field off. Even for this large set, however, alignment at the module level is problematic due to the limited number of muon tracks intersecting any given module. The alignment was, therefore, only performed at the level of TOB rods and TIB strings and not at the level of single module as it was done, in the previous section, using data taken in the tracker assembly hall (but even then, only few modules could be aligned to such precision using the overlaps). The survey information (section 5.2.1) was used as a starting point, thus providing some knowledge of the alignment at the module level. 
Table 15. Average local shifts applied to the sub-structures of TIB and TOB by the alignment algorithm.

\begin{tabular}{|l|c|c|c|}
\hline & $\langle\Delta u\rangle[\mathrm{mm}]$ & $\langle\Delta w\rangle[\mathrm{mm}]$ & $\langle\Delta \gamma\rangle[\mathrm{mrad}]$ \\
\hline TOB & 9.91 & N/A & 7.2 \\
TIB & 0.31 & 1.7 & 0.76 \\
\hline
\end{tabular}

Table 16. Most sensitive track quantities for three different alignment conditions. All the numbers are evaluated for tracks with hits in 3 or more layers.

\begin{tabular}{|c|c|c|c|c|c|c|c|}
\hline $\begin{array}{c}\text { Alignment } \\
\text { status }\end{array}$ & $\begin{array}{c}\text { \# rec. } \\
\text { tracks }\end{array}$ & $\left\langle\chi^{2}\right\rangle$ & $\langle$ \#of hits $\rangle$ & $\begin{array}{c}\text { res. TIBL2 } \\
\text { mono }[\mu \mathrm{m}]\end{array}$ & $\begin{array}{c}\text { res. TIBL3 } \\
\text { mono }[\mu \mathrm{m}]\end{array}$ & $\begin{array}{c}\text { res. TOBL1 } \\
\text { mono }[\mu \mathrm{m}]\end{array}$ & $\begin{array}{c}\text { res. TOBL5 } \\
\text { mono }[\mu \mathrm{m}]\end{array}$ \\
\hline No alignment & 1460 & 20.1 & 3.3 & 526 & 416 & 2660 & 1986 \\
\hline Prel. alignment & 3263 & 16.5 & 4.0 & 518 & 387 & 1547 & 1999 \\
\hline Alignment w/o survey & 4894 & 6.5 & 4.3 & 208 & 135 & 389 & 710 \\
\hline Alignment w/survey & 4956 & 6.0 & 4.3 & 177 & 125 & 357 & 687 \\
\hline
\end{tabular}

In addition, the MTCC tracker geometry was not optimal from an alignment point of view. The TOB data, for example, suffer from a small number of layers, a limited range of track angles and a large lever arm. The barrel alignment was then done in two steps: 1) alignment of TOB rods with TIB strings fixed, assuming the internal TIB misalignment was small compared to that of the TOB due to better survey information; 2) alignment of TIB strings with TOB rods adjusted based on the results of step one.

In the first step (the alignment of TOB rods) the free parameters are the position in the measured coordinate (local $u$ coordinate) and the rotation around the radial axis (local $\gamma$ rotation). In the second step (the alignment of TIB strings) the free parameters are the local $u$ coordinate, the local $\gamma$ rotation and the radial coordinate (local $w$ coordinate).

The total movements applied by the algorithm are summarized in table 15 . The same alignment procedure was run without using the survey information.

As can be seen in table 16, a clear improvement of the track quality with alignment is observed. The input of survey measurements also improves the quality of track reconstruction by adding individual module information. In order to account for remaining misalignment, the hit error is increased by $100 \mu \mathrm{m}$.

\subsubsection{Track reconstruction results}

The numbers of reconstructed tracks after applying the alignment corrections for both the Cosmic Track Finder and the Road Search Algorithm are given in table 17.

The distributions of the most interesting quantities of reconstructed cosmic muon tracks in the $B=3.8 \mathrm{~T}$ data sample are shown in figure 54. The smaller number of reconstructed tracks of the Road Search algorithm is due to seeding generation, which requires an inner hit in TIB layer 2 and an outer hit in TOB layer 1 or 5, resulting in a limited geometrical acceptance: tracks with, for example, hits in TIB layer 3 and the two TOB layers are not reconstructed. As a cross-check, these hit requirements have been applied to the Cosmic Track Finder, and this resulted in a comparable number of reconstructed tracks. 
Table 17. Number of reconstructed tracks for the Cosmic Track Finder and the Road Search algorithm in the different data samples. The smaller number for the Road Search algorithm is the result of a limited geometrical acceptance.

\begin{tabular}{|c|c|c|c|}
\hline & $B=0.0 \mathrm{~T}$ & $B=3.8 \mathrm{~T}$ & $B=4.0 \mathrm{~T}$ \\
\hline Cosmic Track Finder & 5108 & 3588 & 583 \\
\hline Road Search & 4737 & 2343 & 267 \\
\hline
\end{tabular}

Apart from the different numbers of reconstructed tracks, the two track algorithms lead to similar performance. The $\phi$ distributions show a peak around $-\pi / 2$, being compatible with tracks that originate from the top of the detector and travel outside in. Both $\phi$ and $\eta$ distributions of the two algorithms are compatible with the trigger layout. The different geometrical acceptance of the two algorithms affects also the measured $p_{T}$ spectrum. The number of hits per track is smaller for the Road Search, since the algorithm uses matched RecHits instead of $r$ - $\phi$ and stereo RecHits separately.

\subsubsection{Comparison of tracks reconstructed in the tracker and in the muon chambers}

To verify the track reconstruction performance, a comparison of tracks reconstructed by the Cosmic Track Finder and tracks reconstructed in the muon chambers was performed. The track direction was calculated both in the uppermost tracker layer hit by the muon and in the innermost available Drift Tube chamber.

Figure 55 shows the correlation of the direction measured in the absence of magnetic field. The width of the difference $\phi_{t k}-\phi_{D T}$ is about $25 \mathrm{mrad}$. The poor resolution in $\eta$ direction is the cause of the large spread in $\eta$ correlation. The spread is significantly reduced by selecting tracks with hits in all layers.

For events with the magnetic field, as expected, the difference of $\phi_{t k}-\phi_{D T}$ (figure 56) decreases with increasing transverse momentum and it has different sign for positive and negative muons. The measured charge occasionally happens to have the sign opposite to that expected from the difference between the phi measured in the tracker and the phi measured in the muon drift tubes. This charge flip occurs because the tracker tracks are made of three hits only, which makes their measured properties very sensitive to residual misalignment and to noise. The $\eta$ correlation is similar for tracks reconstructed without magnetic field. To perform a more quantitave comparison it would have been necessary to propagate the track from the tracker to the muon system or viceversa. Unfortunately, the uncertainty on the measured momentum, both in the tracker and in the muon system, combined with the amount of material traversed by the muon and the lack of knowledge on the relative position of the two subdetector, made a quantitative comparison impossible.

\subsection{TIB and TOB performance}

The performance of TIB and TOB modules were assessed by analysing the properties of reconstructed clusters, either associated or not to reconstructed tracks. During data taking all modules were kept at bias voltage of $200 \mathrm{~V}$, above the point where full depletion of the silicon bulk is achieved. The APV chip was operated in peak mode and zero suppression was performed in the FED. 

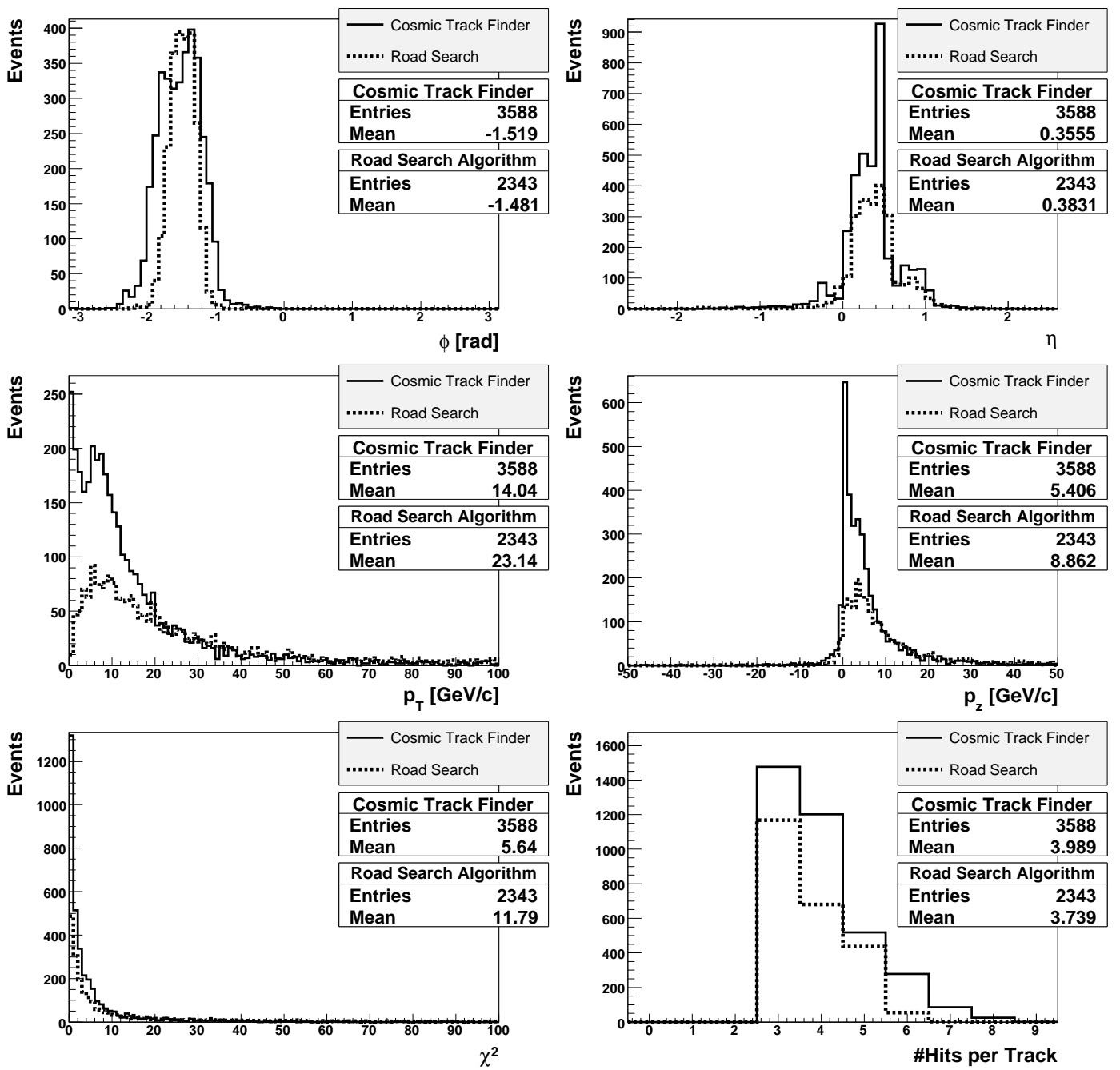

Figure 54. Various track distributions for the Cosmic Track Finder (solid line) and the Road Search algorithm (dashed line) using the $B=3.8 \mathrm{~T}$ data sample: $\phi$ and $\eta$ (top), $p_{T}$ and $p_{z}$ (middle), $\chi^{2}$ and number of hits per track (bottom). The distributions for the Cosmic Track Finder are shown for tracks with hits in at least three layers. 

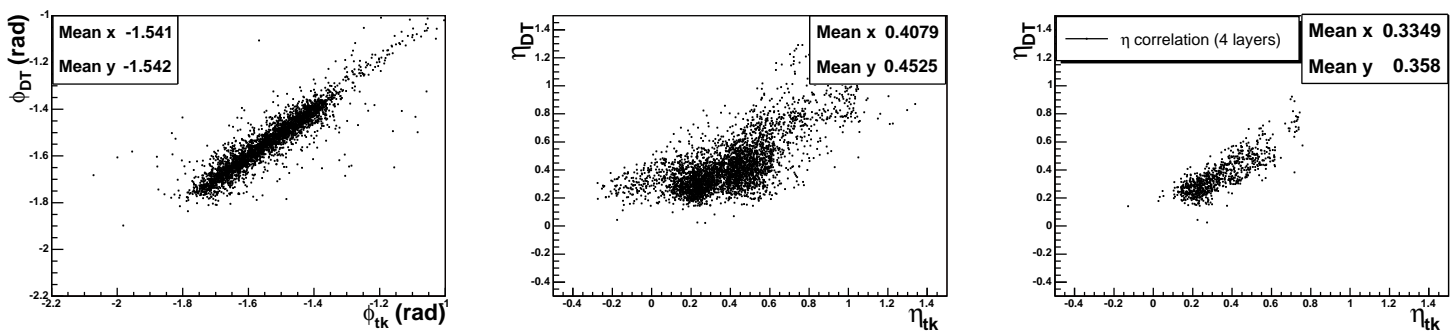

Figure 55. Correlations of directions of tracks, in the absence of magnetic field, reconstructed in the tracker and in the Drift Tubes. The $\phi$ correlation is shown left. The $\eta$ correlation for all the tracks (for tracks with hits in 4 layers) is shown in the centre (right).
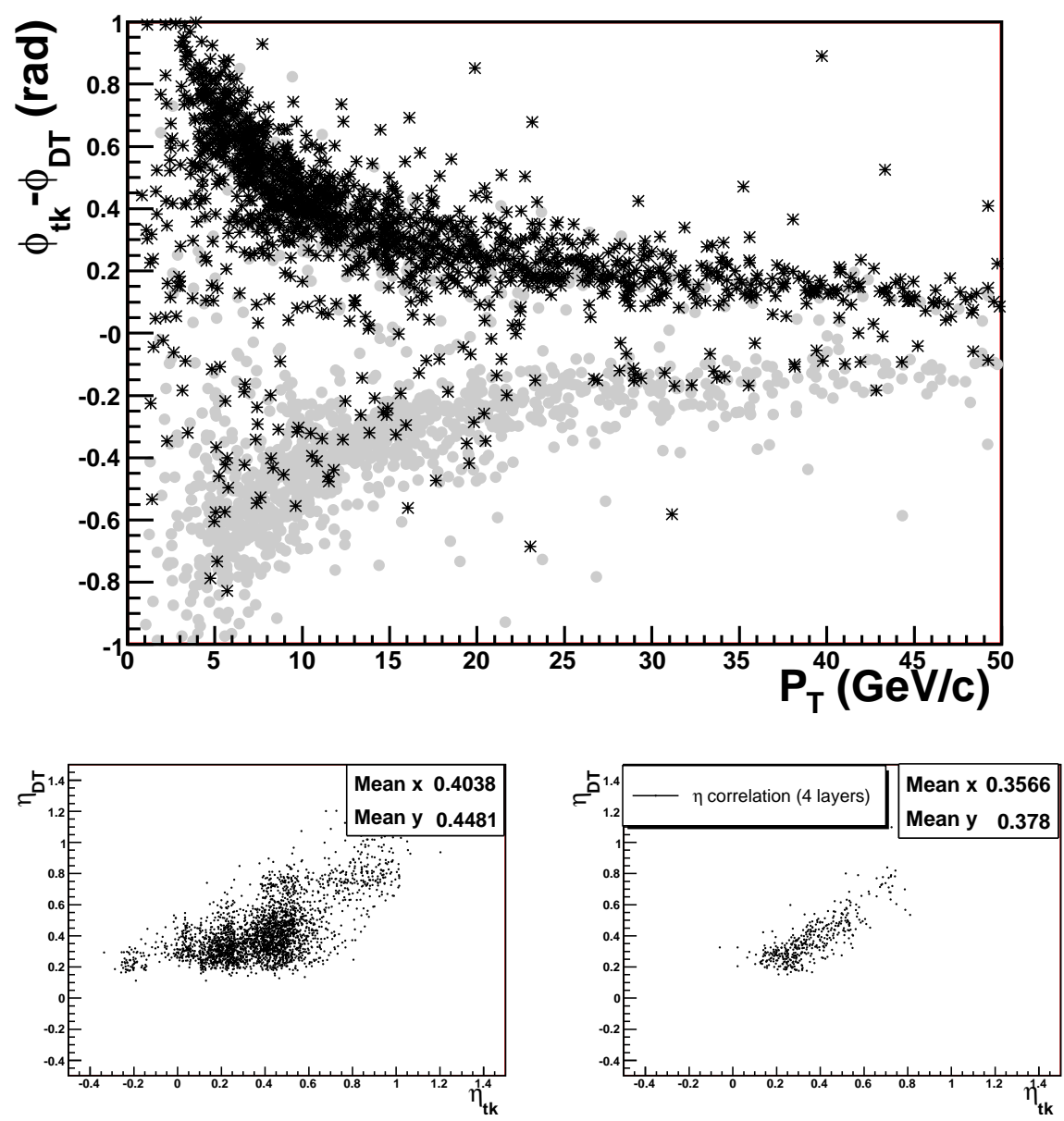

Figure 56. Correlations in the direction of tracks, reconstructed either in the tracker or in the Drift Tubes, at $B=3.8 \mathrm{~T}$. The difference $\phi_{t k}-\phi_{D t}$ (top) is correlated to the transverse momentum measured by the tracker for positive (black) and negative (gray) muons. The bottom plots show the $\eta$ correlation for all the tracks (left) and for tracks with hits in four layers (right). 

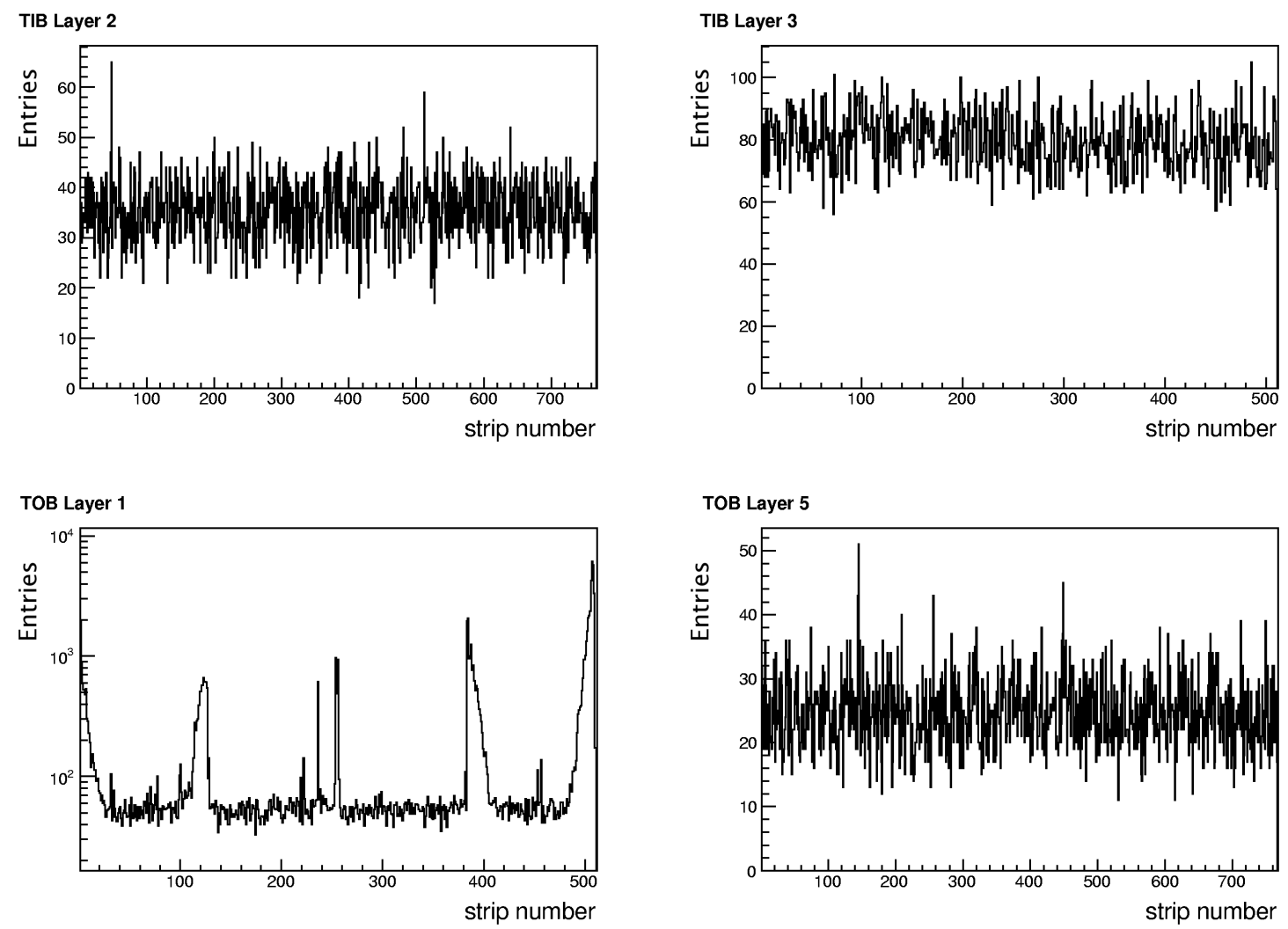

Figure 57. Cluster position for TIB and TOB at $B=0 \mathrm{~T}$.

The position inside the module of all reconstructed clusters, in events with one hit in at least three different TIB and TOB layers, is shown in figure 57. The distributions are all uniform, with the exception of TOB layer 1, where an excess at chip edge is present because of "wing noise" described earlier. This uniformity is a further proof that the number of noisy strips was small, although the modules used in the MTCC were of pre-production grade. The number of noisy channels was consistent with results from module production tests and stable with time.

The charge of clusters generated by cosmic muons is proportional to the length of the path in the sensitive volume of the detector. Slanted particle will therefore generate a cluster of higher charge. If the cluster is correctly associated to the reconstructed track it is however possible to rescale the cluster charge to the traversed detector thickness. In figure 58 the resulting charge distribution is shown together with the results of the fit to a Landau function convoluted with a Gaussian function. The most probable value of the Landau (MP in the plot) is indeed proportional to the module thickness, while the sigma of the Gaussian (GSigma) account for the uncertainty in the track direction and the spread in the opto-hybrid gain value, as will be discussed in section 5.5.

The distribution of the corrected cluster charge versus strip position is shown in figure 59. The only deviation from a uniform distribution is an excess of low charge clusters in TOB layer 5, due to a faulty module. A small excess around the horizontal line at 250 may also be visible. This excess is due to the data processing in the FED, which works with 10 bits ADC range, but is limited in zero-suppressed mode to 8 bits. To avoid, at least partially, the loss of information, 1023 ADC 

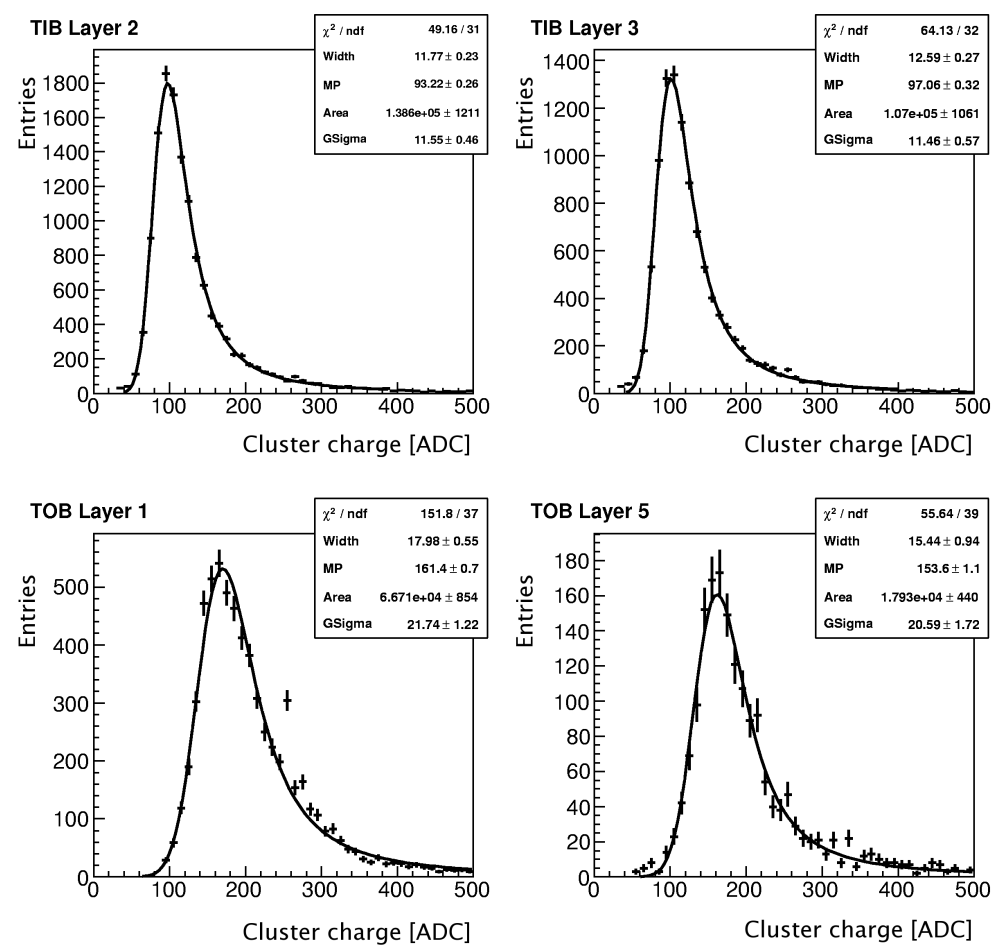

Figure 58. Cluster charge for TIB and TOB modules at $B=0 \mathrm{~T}$. The cluster charge is proportional to the detector thickness: nominally $320 \mu \mathrm{m}$ for TIB and $500 \mu \mathrm{m}$ for TOB, though in both cases the active sensor thickness is $20-30 \mu \mathrm{m}$ less.
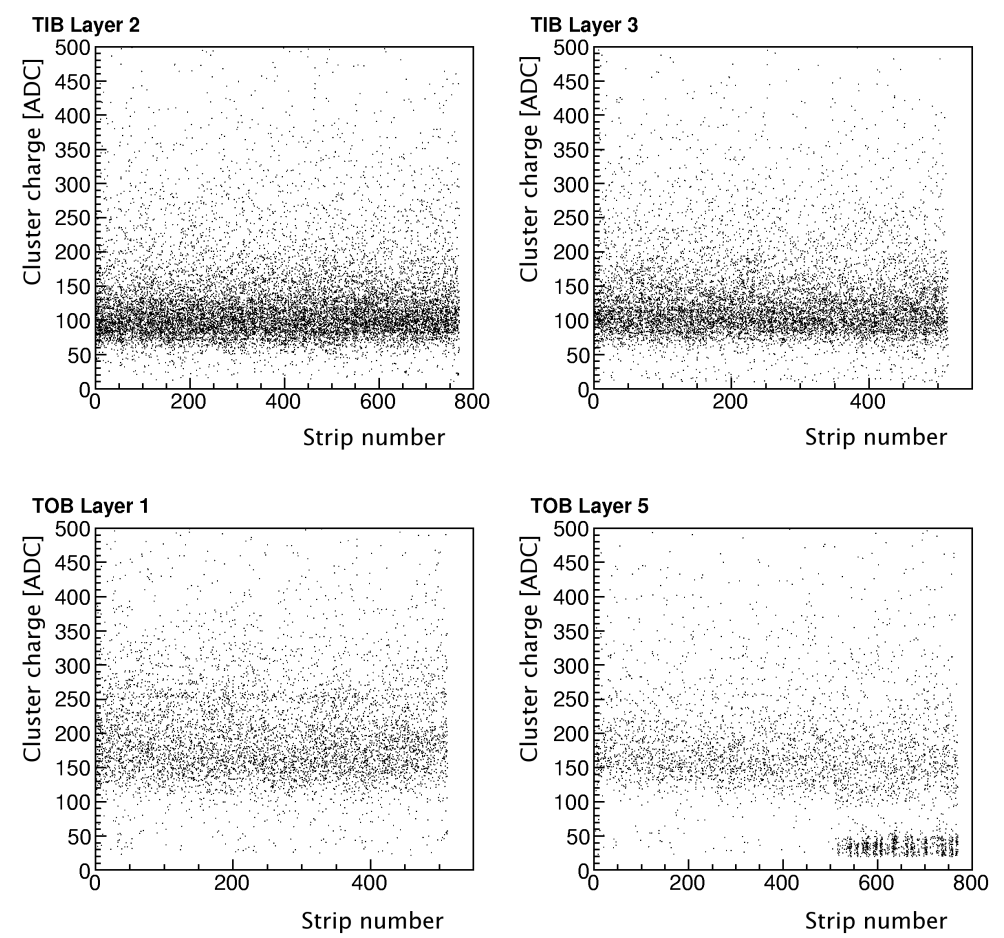

Figure 59. Cluster charge vs. strip number for TIB and TOB at $B=0 \mathrm{~T}$. 

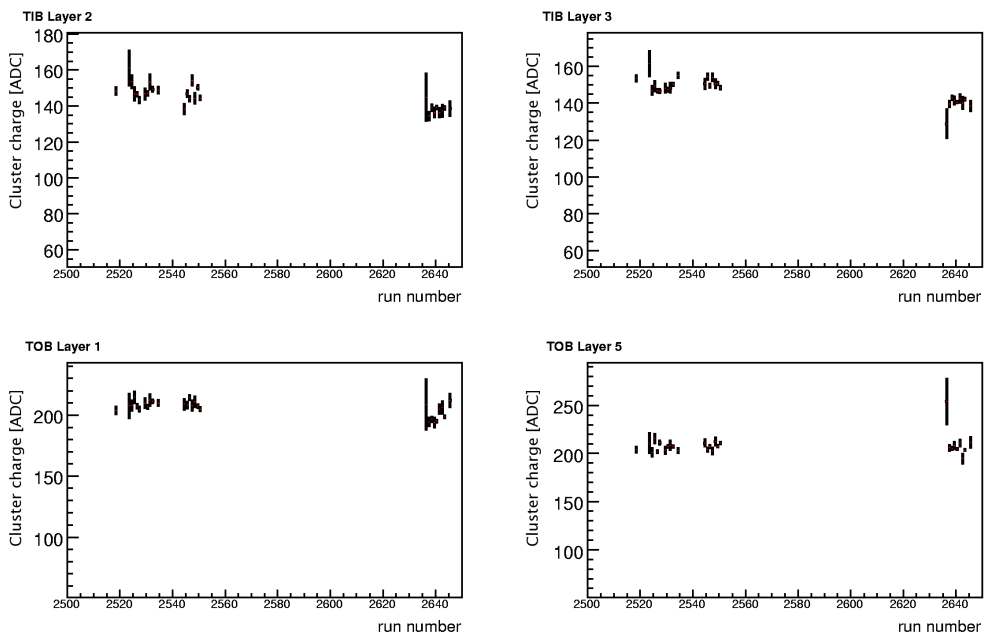

Figure 60. Cluster charge for TIB and TOB vs. run number at $B=0 \mathrm{~T}$.
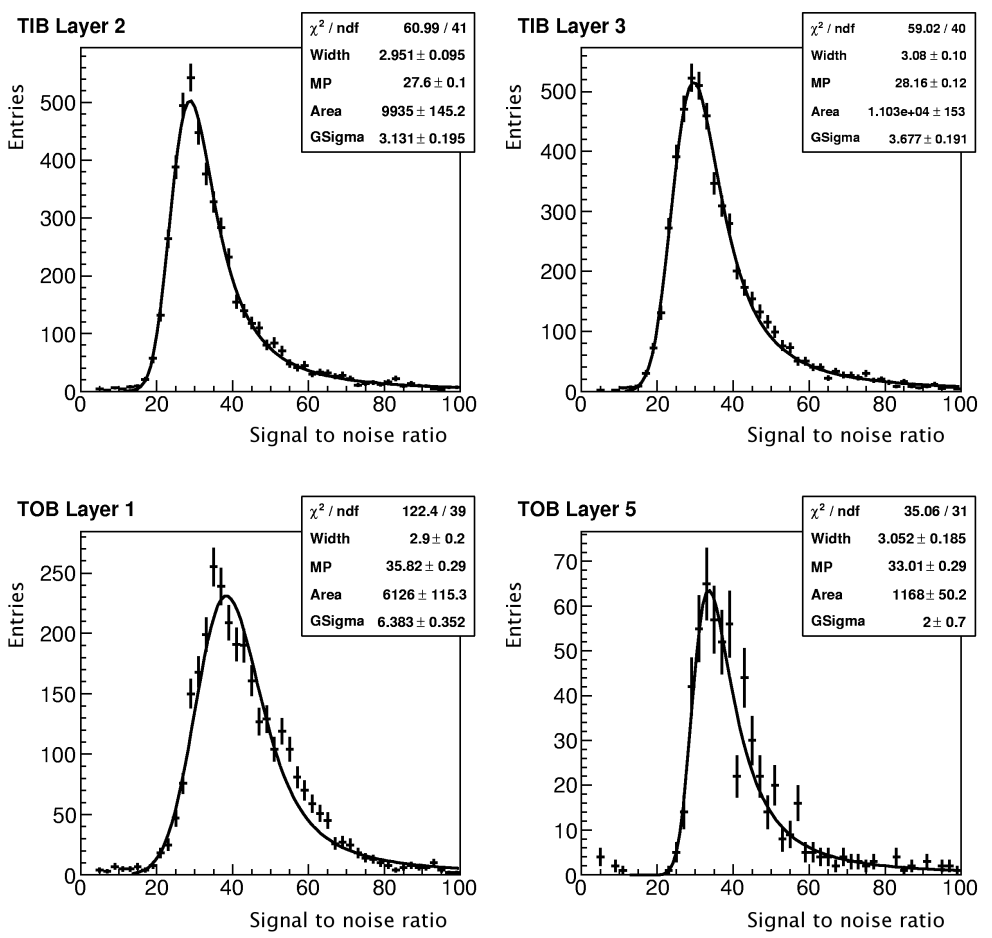

Figure 61. Signal-to-noise ratio distributions of clusters for TIB and TOB at $B=0 \mathrm{~T}$ corrected for the path length in silicon.

value is set to 255 , values from 254 to 1022 are set to 254 , while values from 0 to 253 are left unchanged. As can be seen from figure 60, cluster charge also showed good stability across the entire data taking period.

Signal-to-noise ratio distributions, again corrected for the path length, together with results of the fit to a Landau function convoluted with a Gaussian function are presented in figure 61. The 

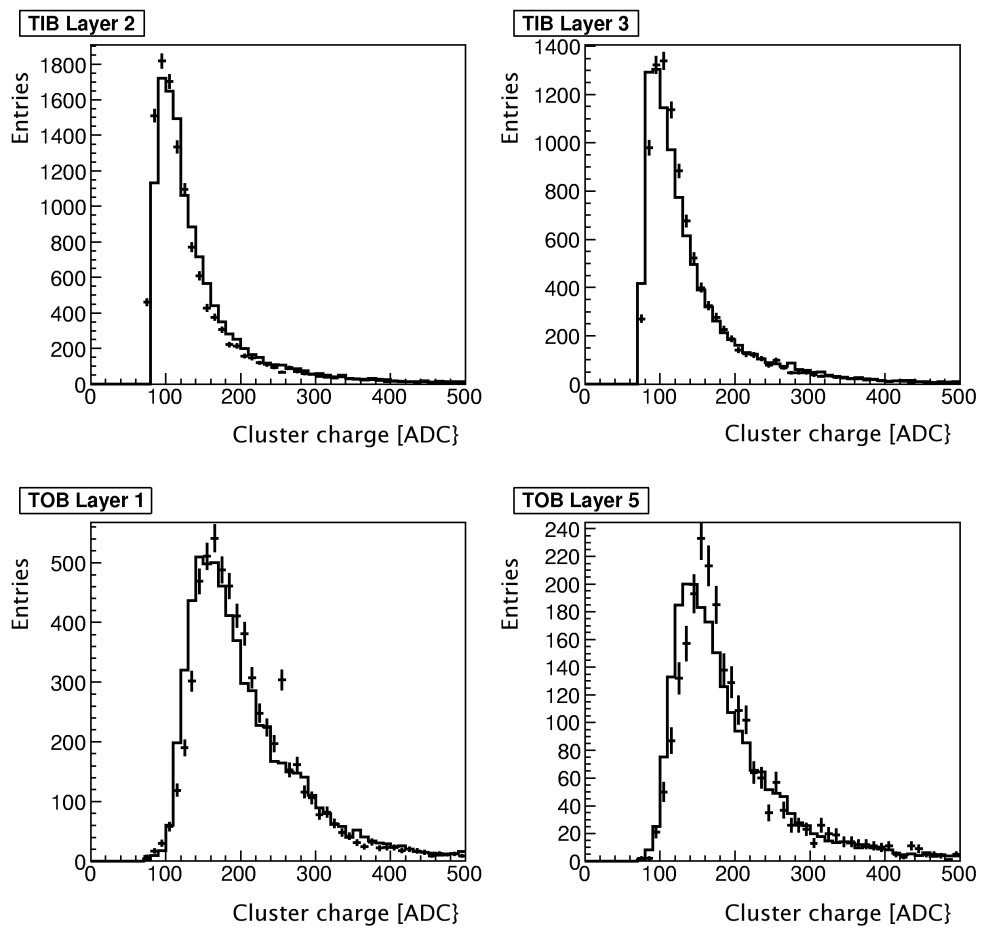

Figure 62. Cluster charge distributions for TIB and TOB at $B=0 \mathrm{~T}$. Solid line - MC simulation, points data.

noise of the cluster is defined here as $\sigma_{\text {cluster }}=\sqrt{\sum_{i} \sigma_{i}^{2} / N_{\text {strips }}}$, where $\sigma_{i}$ is the noise of strip $i$ measured in the pedestal run and the sum runs over all strips in the cluster. The quadratic sum is divided by the number of strips in the cluster, $N_{\text {strips }}$. Therefore, on average, this cluster noise is equal to the single strip noise, independently of the cluster size. A $S / N$ ratio of about 28 for the TIB and 33 for the TOB was measured in peak mode. The results indicate excellent performance of these tracker substructures.

In figure 62, the cluster charge distributions are compared to the distribution in simulated events. The only parameter that could be adjusted to data is the number of ADC counts per released charge in the silicon bulk. This conversion factor was set to $250 e^{-} / \mathrm{ADC}$ count. The agreement between real data and simulation shows that the particle interaction with the material and the detector response are well understood.

A more detailed investigation of the charge distribution within a cluster can be the obtained from the response function, $\eta_{\text {res }}$. To build this function, in each cluster, the strip with the highest signal and the adjacent strip with the highest signal are selected (whether or not they also belong to the cluster). Of these two strips the one with the smaller strip number is referred to as the left strip and the other one as the right strip. The response function is then defined as

$$
\eta_{\text {res }}=\frac{Q_{\text {left }}}{Q_{\text {left }}+Q_{\text {right }}}
$$

where $Q_{\text {left }}\left(Q_{\text {right }}\right)$ is the charge of left (right) strip in the cluster. By definition $\eta_{\text {res }}$ is distributed between 0 and 1 . Ionization deposited in the silicon bulk by the charged particle can be shared 

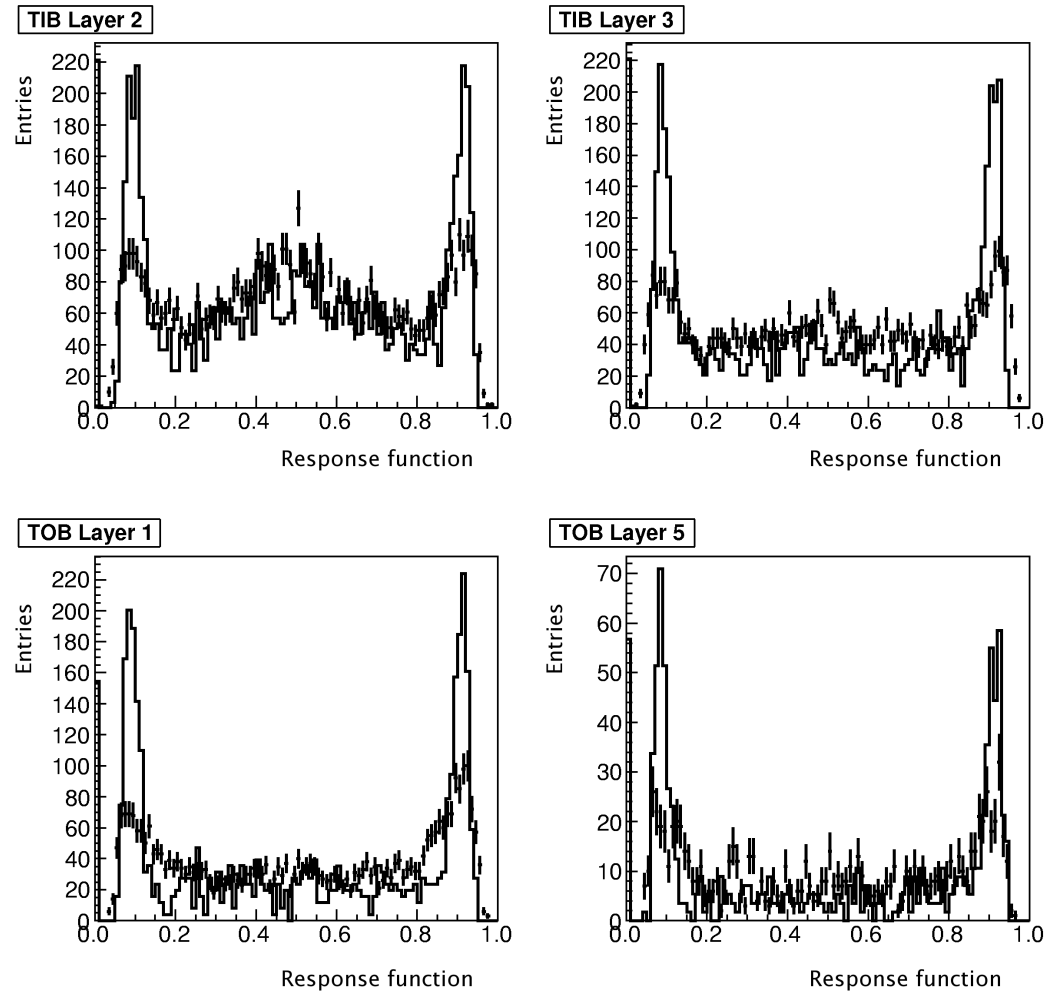

Figure 63. Cluster response function $\eta_{r e s}$ for TIB and TOB for $B=0$ T. Solid line - MC simulation, points - data.

between several strips, which then form a cluster with more than one strip. In this case $\eta_{\text {res }}$ has a fairly smooth distribution between 0 and 1 . But $\eta_{\text {res }}$ is also sensitive to charge coupling between the adjacent strips. If the signal is not shared between several strips and the entire ionization charge is deposited on the single strip $\left(Q_{1}\right)$, the adjacent strips $\left(Q_{2}\right.$ and $\left.Q_{3}\right)$ can still have a non-zero signal because of the cross-talk $(x)$ :

$$
Q_{2}=x Q_{1}, Q_{3}=x Q_{1}
$$

As a result the signal on the central strip is reduced to $(1-2 x) Q_{1}$. Thus, the response function for such clusters is equal to

$$
\eta_{\text {res }}=\frac{x}{1-x}
$$

if the largest induced signal happens to be to the left of the central strip or

$$
\eta_{\text {res }}=\frac{1-2 x}{1-x}
$$

otherwise. This effect results in a two-prong distribution of $\eta$, clearly visible in figure 63 for all clusters.

This distribution is especially sensitive to the charge among adjacent strips. The value of the cross-talk $(x)$ can be extracted by isolating the left peak in $\eta_{\text {res }}$ distribution for single-strip clusters (figure 64) and by fitting it to a Gaussian shape. If $\eta_{\text {res } 1}$ is the mean of the Gaussian distribution for 

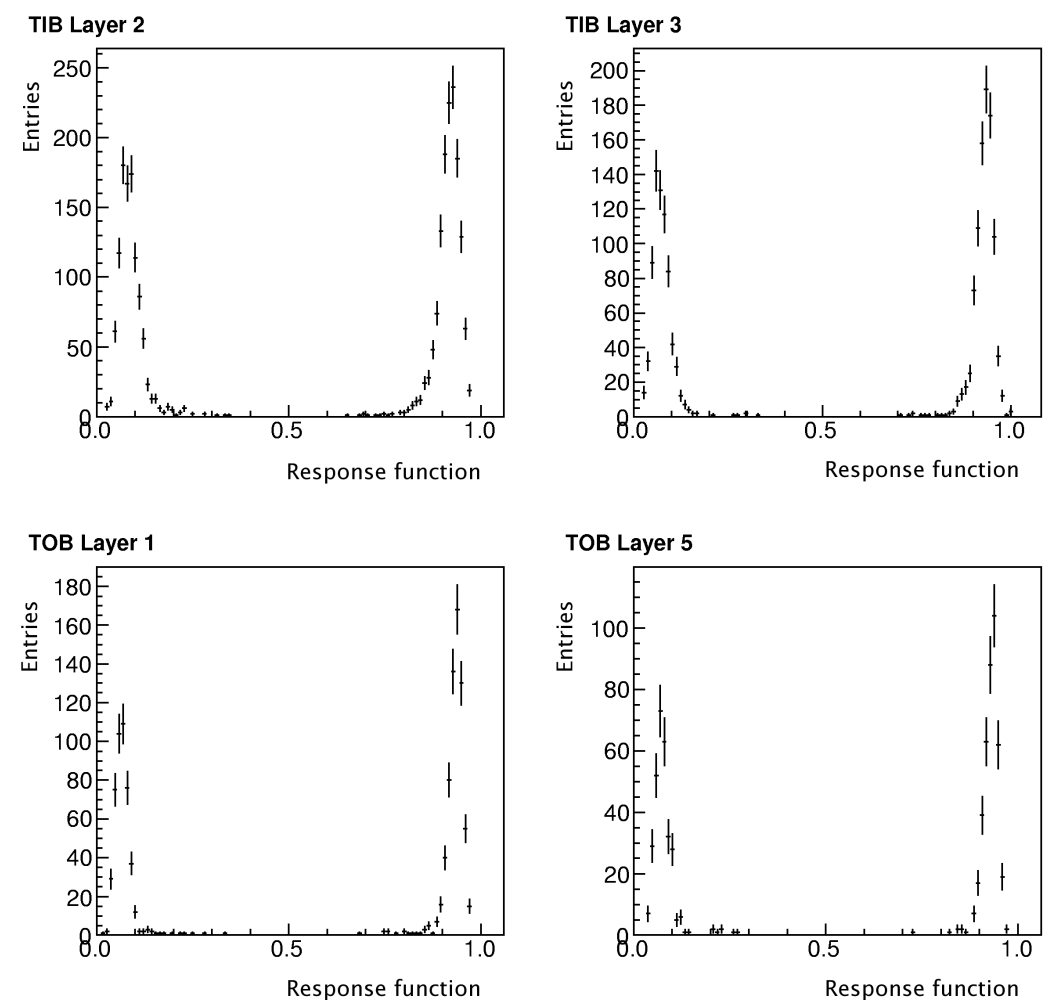

Figure 64. Cluster response function $\eta_{\text {res }}$ for TIB and TOB for single strip clusters, $B=0 \mathrm{~T}$.

left-prong single-strip clusters, then the cross-talk $x$ has the value of

$$
x=\frac{\eta_{\mathrm{res} 1}}{1+\eta_{\mathrm{res} 1}},
$$

From the fit, the value of cross-talk was determined to be $7.8 \pm 2.3 \%$ for TIB L2, $6.7 \pm 2.1 \%$ for TIB L3, $6.2 \pm 1.6 \%$ for TOB L1, $6.6 \pm 1.9 \%$ for TOB L5. The width of the peaks in $\eta_{\text {res }}$ distribution is determined by the readout noise, which is the main component in the single strip noise.

Despite the tuning of the cross-talk in the Monte Carlo simulation, the two-prong distribution in figure 63 is more pronounced in simulated events than in the data. This disagreement is not fully understood, but it may be due to a difference in the distribution of the tracks inclination on the modules. Unfortunately statistics was not enough to perform a more refined study, e.g. as a function of cluster strip multiplicity.

The strip multiplicity is determined by several factors, such as track inclination, magnetic field effects, strip pitch, sensor thickness, and cross-talk. Cluster noise and strip multiplicity for runs taken with the field off are shown in figure 65 and figure 66. Distributions are similar for runs with the field on. In figure 67 the cluster size distribution in data with $B=3.8 \mathrm{~T}$ is compared to simulated events, showing a resonable agreement. 

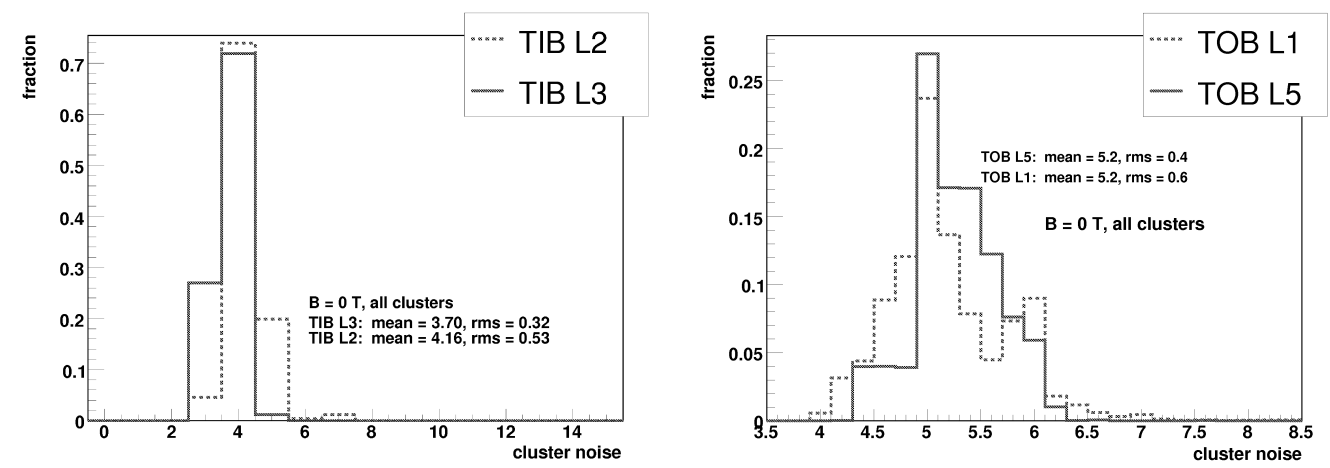

Figure 65. Cluster noise for TIB and TOB at $B=0 \mathrm{~T}$.
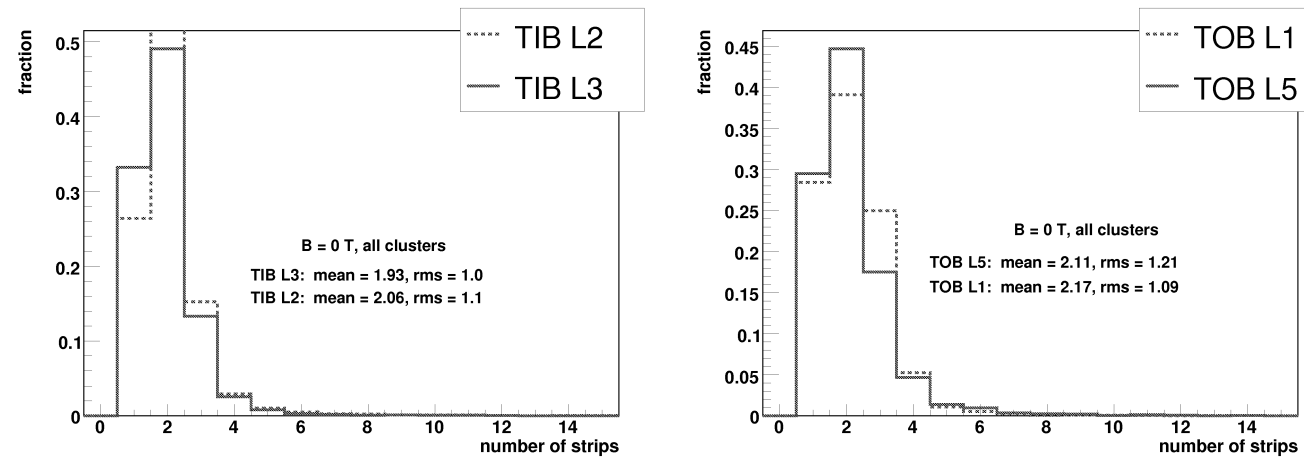

Figure 66. Cluster size for TIB and TOB at $B=0 \mathrm{~T}$.
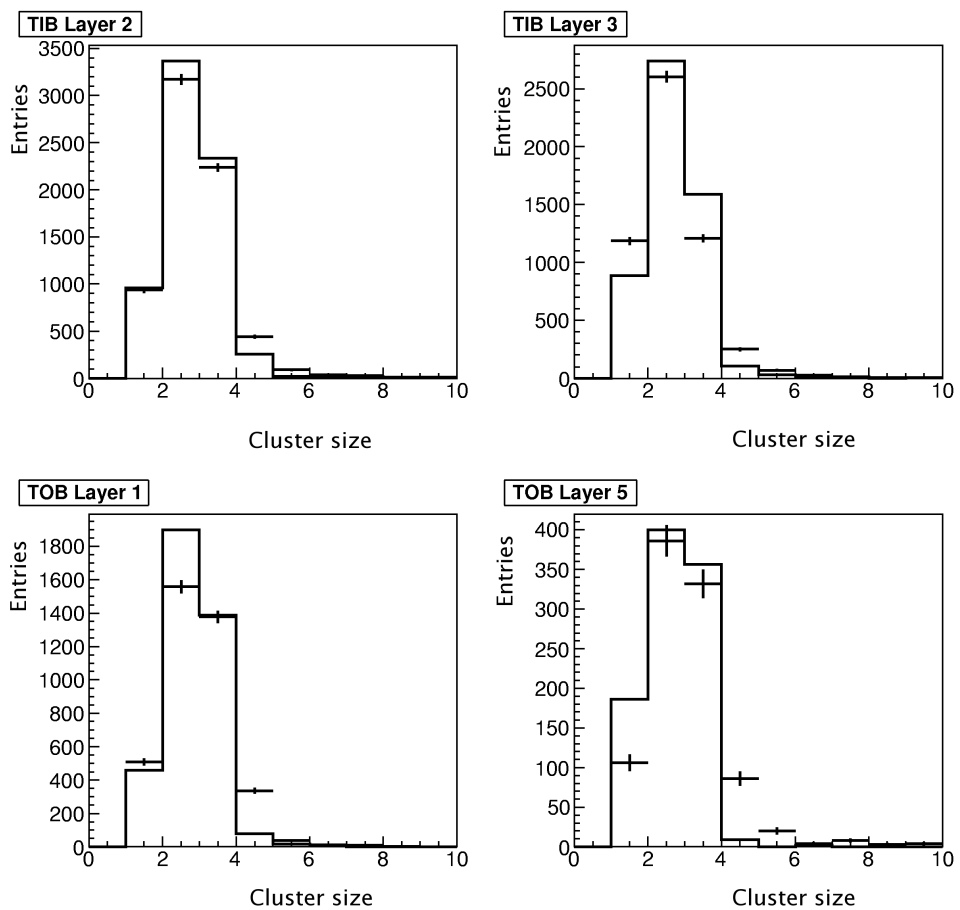

Figure 67. Cluster size distributions for TIB and TOB at $B=3.8 \mathrm{~T}$. Solid line - MC simulation, points data. 


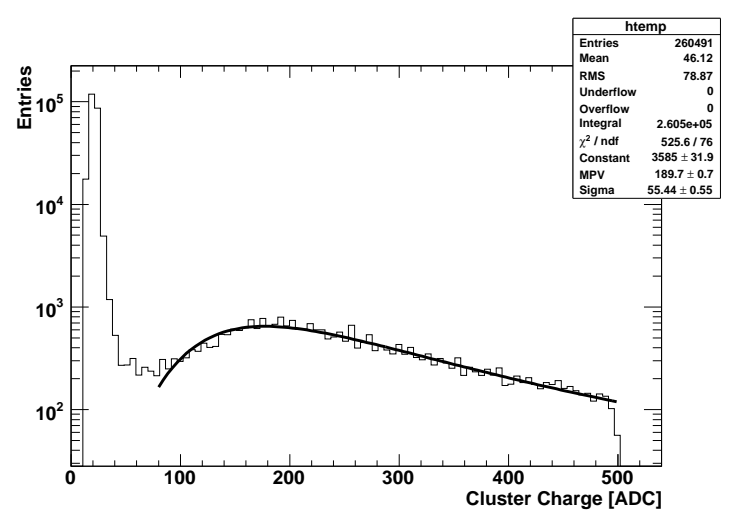

Figure 68. Cluster charge for TEC modules at $B=$ $3.8 \mathrm{~T}$ (cluster charge $<500$ ). The result of the fit with a Landau function is shown.

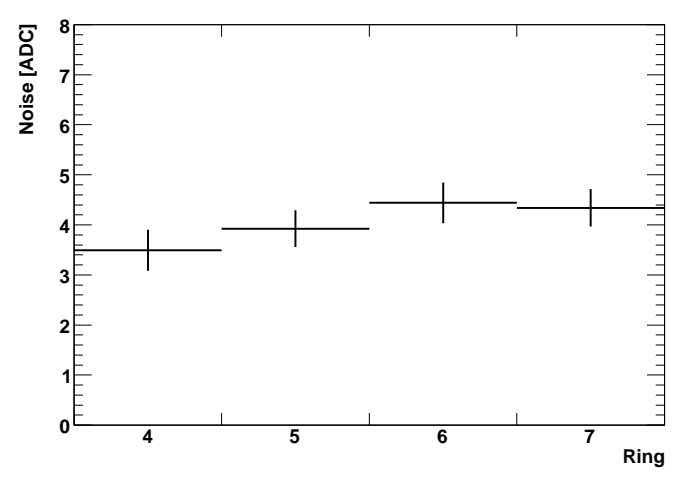

Figure 70. Uncalibrated cluster noise as a function of TEC ring number $(\mathrm{B}=3.8 \mathrm{~T})$.

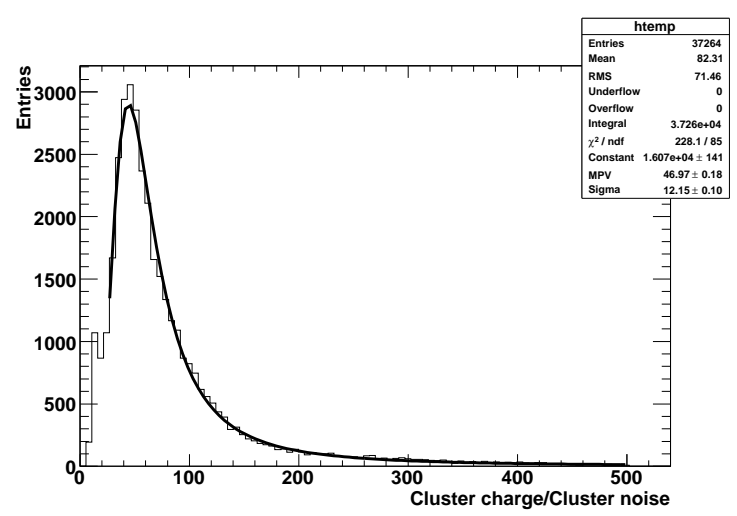

Figure 69. Cluster charge over cluster noise for TEC with $\mathrm{S} / \mathrm{N}>10, B=3.8 \mathrm{~T}$. The result of the fit with a Landau function is shown.

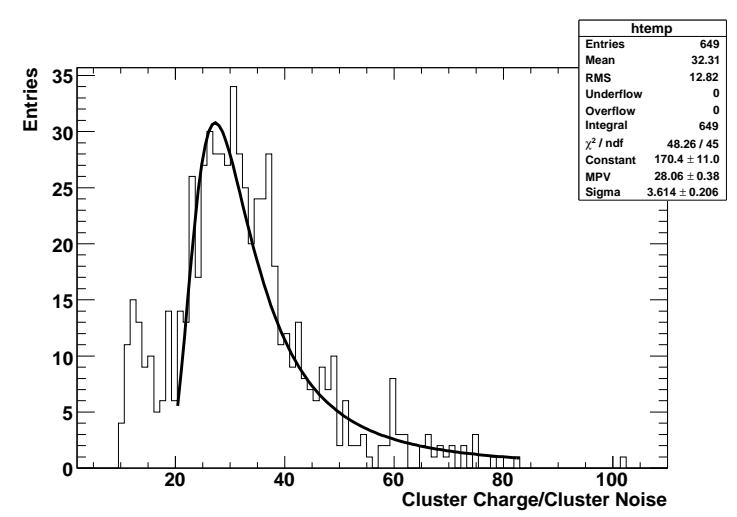

Figure 71. Cluster charge over cluster noise for TEC ring 4 single strip clusters with $\mathrm{S} / \mathrm{N}>10$ $(\mathrm{B}=3.8 \mathrm{~T})$. The result of the fit with a Landau function is shown.

\subsection{TEC performance}

As described earlier, in the MTCC configuration, the TEC performance can not be studied using tracks, therefore it is not possible correlate basic cluster quantities with track parameters. In figure 68 the distribution of the cluster charge is shown. Cosmic muons hits, peaks at about 200 ADC counts and are clearly separated from the tail of the noise. The apparent peak around 30 ADC counts is due to the signal-to-noise cut at 5 . In the following analysis, this peak has been eliminated by requiring a minimum cluster signal-to-noise of 10 . Furthermore, clusters with a charge of more than 500 ADC counts are ignored.

Figure 70 shows the cluster noise as a function of ring number on the petal. The noise increases with capacitance as a result of increasing strip length for the outer rings.

The signal-to-noise distribution for all clusters is shown in figure 69. The fit with the Landau function yields a Most Probable Value (MPV) of about 47. Such large value is due to tracks which cross the detector with shallow incidence angle. Figure 71 shows instead the signal-to-noise for 

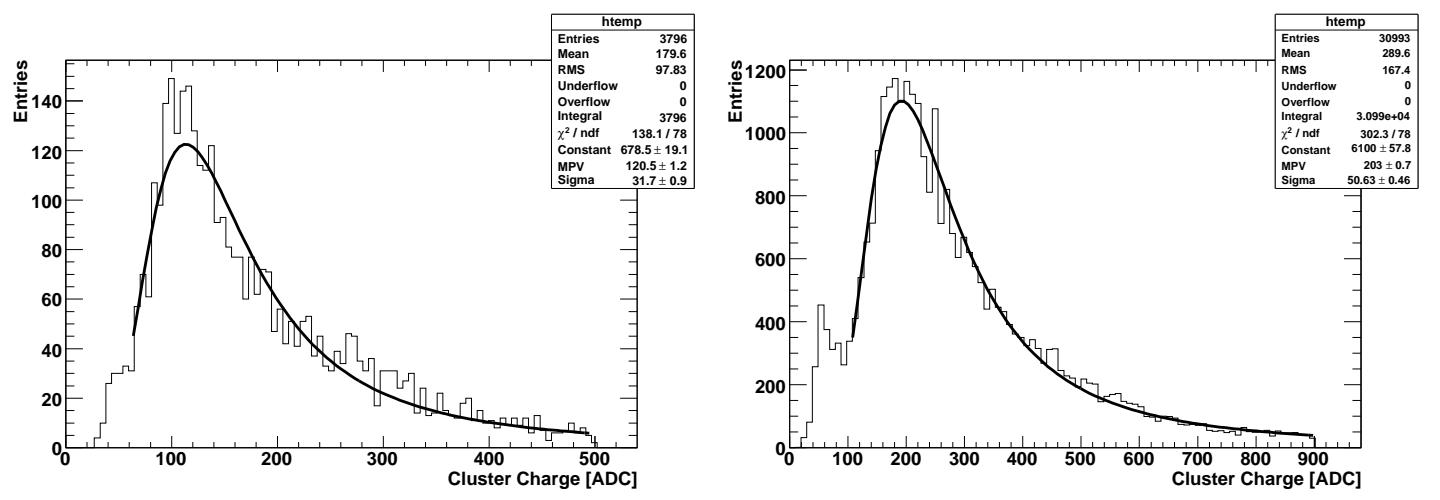

Figure 72. Cluster charge for ring 4 modules (left) and ring 5 to ring 7 modules (right) $(\mathrm{B}=3.8 \mathrm{~T})$. In both cases, the result of the fit with a Landau function is shown.

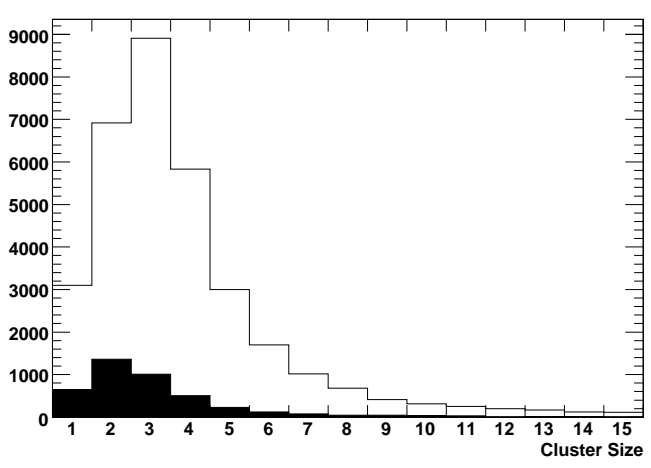

Figure 73. Cluster size for TEC clusters with $\mathrm{S} / \mathrm{N}>10(\mathrm{~B}=3.8 \mathrm{~T}$, black: ring 4 modules, empty histogram: ring 5 to ring 7 modules).

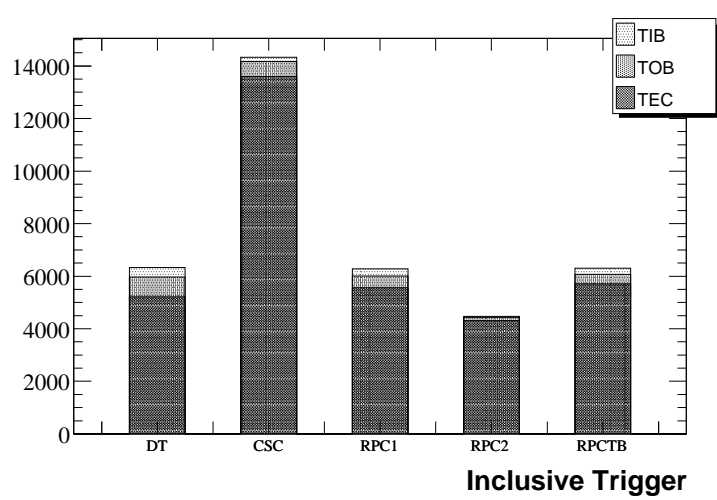

Figure 74. Fired Triggers for $B=0 \mathrm{~T}$.

single strip clusters in ring 4 modules of TEC. The Landau fit yields an MPV of 28, which is consistent with a value of 29 obtained in test beam [37].

Figure 72 shows the cluster charge separately for thin (ring 4) and thick sensors (rings 5-7): nominally the thickness is $320 \mu \mathrm{m}$ and $500 \mu \mathrm{m}$ respectively, though in both cases the active sensor thickness is 20-30 $\mu \mathrm{m}$ less. Most TEC clusters comprise 2 or 3 strips (see figure 73), which is comparable to the cluster sizes obtained for TIB and TOB modules.

A study was made to see if the sample could be enhanced with valid TEC hits by requiring specific triggers to have fired. A detailed analysis of the trigger statistics clearly shows that TEC events are preferably associated with the triggers from the CSC (figure 74). In figure 75, the cluster charge distribution is shown separately for CSC inclusive and DT inclusive triggers and for CSC inclusive and RPCTB inclusive triggers, respectively (section 2.4). Whereas a clear signal distribution is visible for CSC, the other two triggers show only broad background distributions. This effect can be understood from the geometry of the MTCC setup. The CSCs are closest to the 

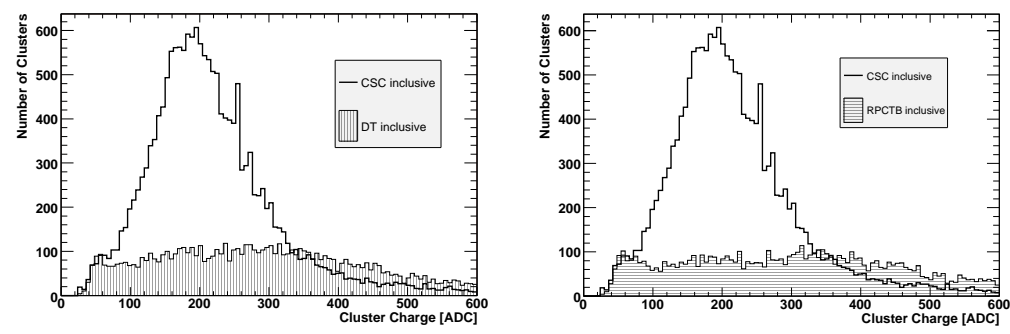

Figure 75. Comparison between CSC and DT trigger (left) or CSC and RPCTB trigger (right), both with a cut on $\mathrm{S} / \mathrm{N}>10(\mathrm{~B}=3.8 \mathrm{~T})$.

TEC modules and mounted parallel to the TEC sensor plane. When requiring at least one hit in a TEC module and a CSC trigger, very few hits in TIB and TOB are left, which again shows that it is not possible to find tracks passing through TEC and TIB/TOB layers simultaneously.

\subsection{Gain measurement}

The charge released in silicon by the passage of a charged particle translates into ADC counts assigned to a set of channels making up a cluster. Non-uniformities in the charge collection and in the readout chain can affect the amplification and linearity of the primary charge. The components involved in this chain are the silicon wafers, the strips, the APV and AOH chips, the optical fibres and the FED.

A significant contribution to possible gain non-uniformities is expected to come from the Linear Laser Driver (LLD) [38] on the AOH. As mentioned in section 4, the LLD has been designed with four gain settings, allowing a certain amount of gain equalization. The residual non-uniformity after optimal settings are applied is still expected to be at the level of $15 \%$.

Even in the presence of a perfectly uniform detector and electronic components, signal height non-uniformities may arise in the signal sampling step because of non-perfect readout synchronization.

A linear and uniform amplification (gain) across all the channels of a silicon module is fundamental for the ultimate space resolution obtainable with these detectors. Also, the performance of the particle identification technique with energy loss in the silicon detectors is sensitive both to the absolute calibration scale and to gain non-uniformities. For this application, uniformity must be guaranteed across the full tracker and not only at the level of individual modules.

The response of part of the signal processing chain was obtained using the height of the digital header produced by each APV. The height of the digital header is not affected by any of the APV settings. The average among the two tick heights $(T H)$ of the APVs connected to each LLD has been measured after optimal gain values were set in the LLD.

Figure 76 shows the distribution of the $\langle T H\rangle / T H$ values of all APV pairs, where $\langle T H\rangle$ is the mean of all responses. The ratio $\langle T H\rangle / T H_{i}$ can be interpreted as the inter-calibration factor to be applied to the signals produced by the $i^{\text {th }}$ APV pair.

The inter-calibrations measured with the tick-height method cannot account for nonuniformities in the silicon, in the amplification chain preceding the LLD, as well as non perfect synchronization of the readout. The ultimate precision on the inter-calibration constants can only 

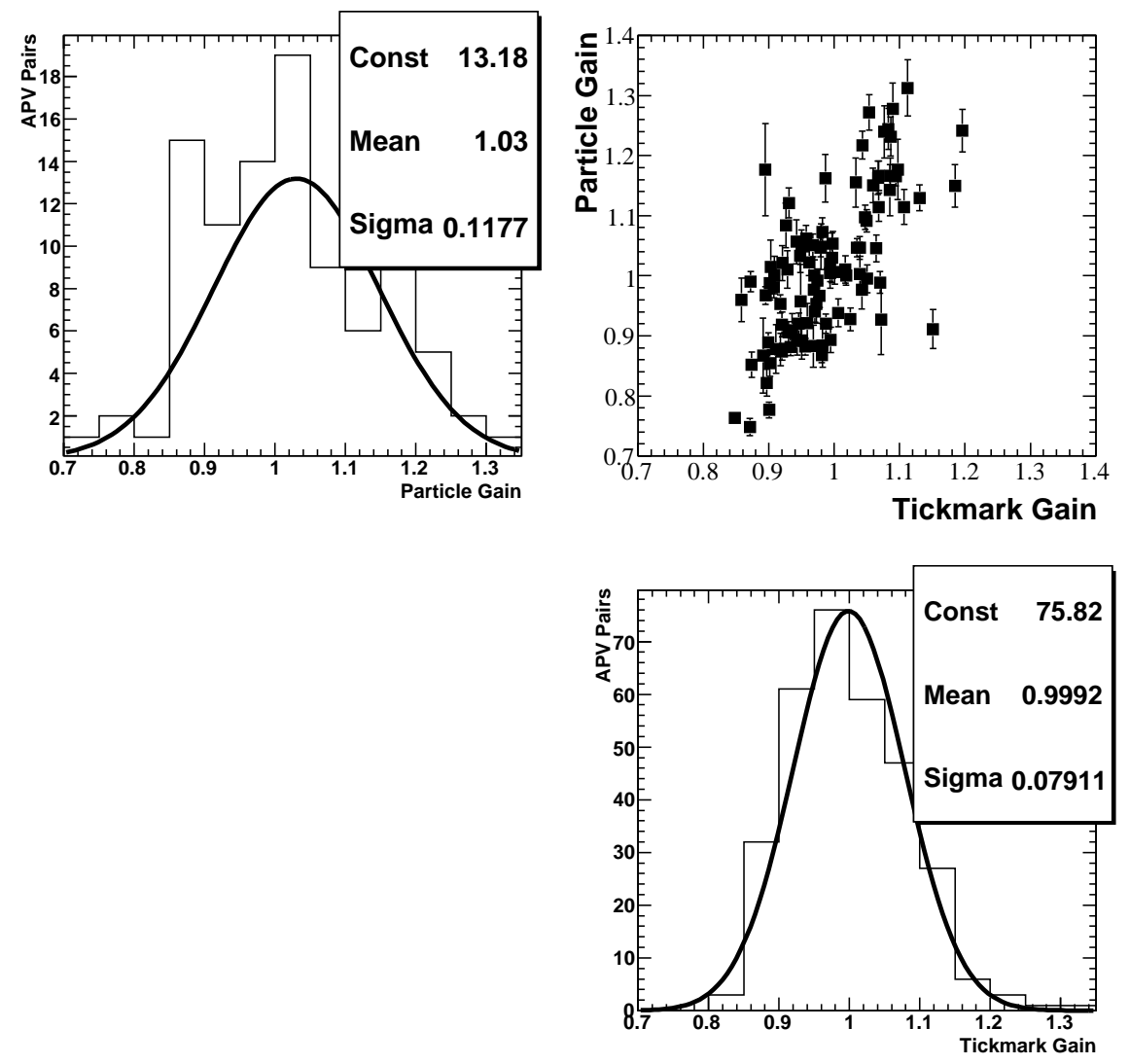

Figure 76. Bottom right: tick height based inter-calibration constants distribution for the APV pairs in the MTCC setup. Top left: particle-based inter-calibration constants distribution for the APV pairs in the MTCC setup. Top right: correlation among the inter-calibration constants computed with the two methods.

be obtained by looking at signals produced by particles. For this reason, the charge of the clusters associated with tracks was used to produce a separate charge distribution for each APV pairs. The charge was normalized to the distance travelled through the active material of the module. This step required the knowledge of the track incidence angle on the detector and the thickness of the sensor.

The obtained distributions were then fit with a Landau curve. The Most Probable Value of the distribution (MP) is used to compute the inter-calibration constant as the ratio MP $/<\mathrm{MP}\rangle$. The distribution of the MP / $<\mathrm{MP}>$ values is shown in figure 76. Only the distributions having at least 60 cluster charge entries and yielding a ratio between $\chi^{2}$ of the fit and the degrees of freedom smaller than 2.0 have been considered for this plot.

It can be noted that the accuracy of this method relies on the assumption that all APV pairs were illuminated with the same muon momentum spectrum. This hypothesis may not be realized due to geometrical reasons. However, the momentum cut of about $3 \mathrm{GeV}$, due to the iron to be traversed by the muons, the exponential fall of the cosmic muon momentum spectrum and the fact that in the range 3 to $6 \mathrm{GeV}$ the energy loss variations are below a few per cent, validate the assumptions that the observed differences are dominated by instrumental effects. 


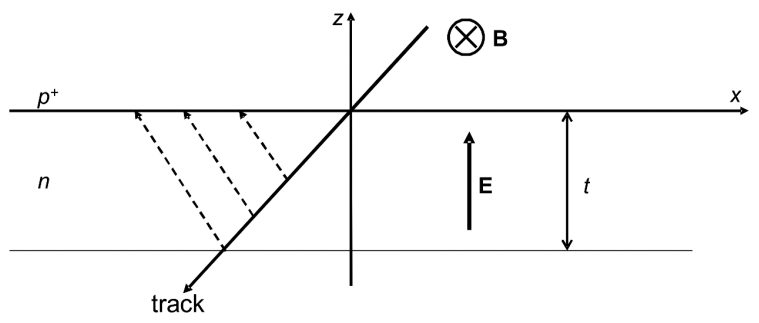

Figure 77. Lorentz drift in the microstrips silicon sensors.
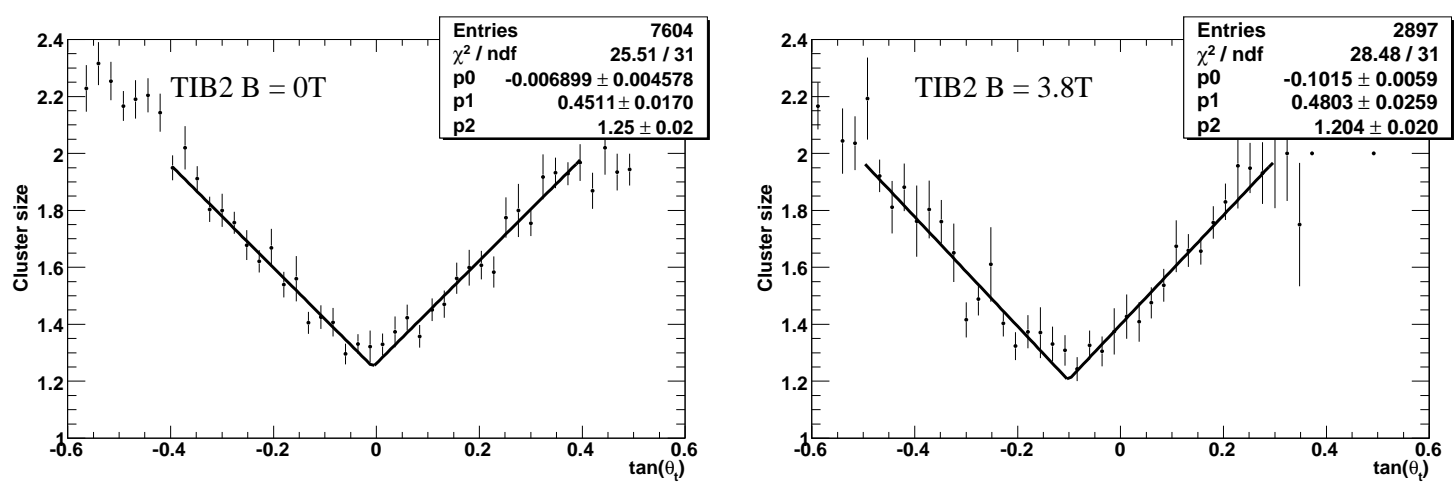

Figure 78. Cluster size versus incident angle in TIB Layer 2 for (a) no B field and (b) $3.8 \mathrm{~T}$. The difference among the minima corresponds to the Lorentz angle.

The correlation between the corrections computed with the two methods, which is relatively good, is shown in figure 76 .

\subsection{Lorentz angle measurement}

It was possible to measure the Lorentz angle [39] in the silicon sensors with reconstructed tracks. The method takes advantage of the fact that the electric field is normal to the strips and therefore, in absence of magnetic field, the drift direction is coincident with the field lines. Hence for normal incidence particles, only one strip is hit, while the cluster size increases with the incidence angle. In the presence of magnetic field, the drift direction is no longer along the electric field drift lines, as shown in figure 77. Therefore the minimal cluster size is found for particles traversing the sensors with the same inclination of the drift lines.

Since the angle between electric field and drift direction is by definition the Lorentz angle, the angle providing a minimal cluster size measures it directly. Two profile plots of cluster size versus the tangent of the incidence angle are shown in figure 78 for TIB Layer 2 for $0.0 \mathrm{~T}$ and 3.8 T respectively.

The function used to determine the minimum is

$$
\text { Size }=\frac{t}{P} \cdot p_{1} \cdot\left|\tan \theta_{t}-p_{0}\right|+p_{2}
$$

where $t$ is the detector thickness, $P$ is the pitch and $p_{0-2}$ the fitted parameters. The most important one, $p_{0}$, is the estimate of $\tan \theta_{L}, p_{1}$ is the slope normalized to the ratio of thickness over pitch and 
Table 18. Measured and expected values of $\tan \theta_{L}$ for $B=3.8 \mathrm{~T}$. Results are shown both for Cosmic Track Finder and Road Search algorithm.

\begin{tabular}{|c|c|c|c|}
\hline \multicolumn{4}{|c|}{$\tan \theta_{L}$} \\
\hline Layer & Measured Cosmic Track Finder & Measured Road Search & Expected \\
\hline TIB Layer 2 & $-0.102 \pm 0.007 \pm 0.012$ & $-0.095 \pm 0.007 \pm 0.001$ & $-0.103 \pm 0.009$ \\
\hline TIB Layer 3 & $-0.075 \pm 0.014 \pm 0.018$ & $-0.082 \pm 0.017 \pm 0.016$ & $-0.103 \pm 0.009$ \\
\hline TOB Layer 1 & $-0.15 \pm 0.02 \pm 0.09$ & $-0.11 \pm 0.04 \pm 0.03$ & $-0.113 \pm 0.011$ \\
\hline TOB Layer 5 & $-0.11 \pm 0.02 \pm 0.01$ & $-0.05 \pm 0.03 \pm 0.02$ & $-0.113 \pm 0.011$ \\
\hline Combined & $-0.105 \pm 0.010$ & $-0.092 \pm 0.007$ & $-0.103 \pm 0.009$ \\
\hline$\chi^{2}$ /n.d.f. & $4.6 / 3$ & $1.8 / 3$ & - \\
\hline
\end{tabular}

$p_{2}$ is the average cluster size in the minimum. Cluster reconstruction thresholds were increased to 5 times the noise value for the strip, 6 for the seed and 7 for the total cluster charge, instead of standard 3, 4, and 5, to remove electronics cross-talk between nearby channels. Because of this cross-talk, in some layers the minimum cluster size was larger than two strips and constant over a wide range of the track incident angles. By increasing the thresholds, strips with very low signals were removed and the cluster width became more sensitive to track angles.

The results on $\tan \theta_{L}$ obtained by the fits for different layers and different tracking algorithm, are summarized in table 18. The first error is the statistical uncertainty of the fit, while the second one is a systematic uncertainty due to alignment precision. This uncertainty was estimated as the difference between the results obtained using the best alignment settings and the settings obtained without survey informations.

The expected values are also shown in table 18. The uncertainty is mainly due to the poor knowledge of the temperature of the detectors during operation. A detailed explanation of the model used for the calculation and the associated uncertainties can be found in ref. [40]. The Lorentz angle depends slightly on the detector thickness. However with the ratio calculated from this model the results of the different layers can be combined as if they were all measured in $320 \mu \mathrm{m}$ thick detector. The results of this combination are also shown in table 18. Both Cosmic Track Finder and Road Search tracks yields a result in agreement with the expected value of $-0.103 \pm$ 0.009 , but the $\chi^{2}$ of the is lower when using RS tracks. Values obtained with RS tracks are shown in figure 79, together with the result of the combination, $\tan \theta_{L}=-0.092 \pm 0.007$.

\section{Conclusions}

The Magnet Test and Cosmic Challenge has been an important experience for the tracker. A slice of tracker detectors, representing about $1 \%$ of the final system, was assembled in a prototype support tube and placed in the superconducting magnet, operated up to its nominal field value of $4 \mathrm{~T}$. The tracking system has been successfully commissioned with a local DAQ and operated in the global DAQ together with all the other subdetectors of CMS. A trigger on cosmic muons was provided, in particular, by the Muon System.

All the main goals identified at the beginning of the MTCC were achieved. It was possible to read out and analyse the data in the new CMS software framework, CMSSW. The Data Quality 


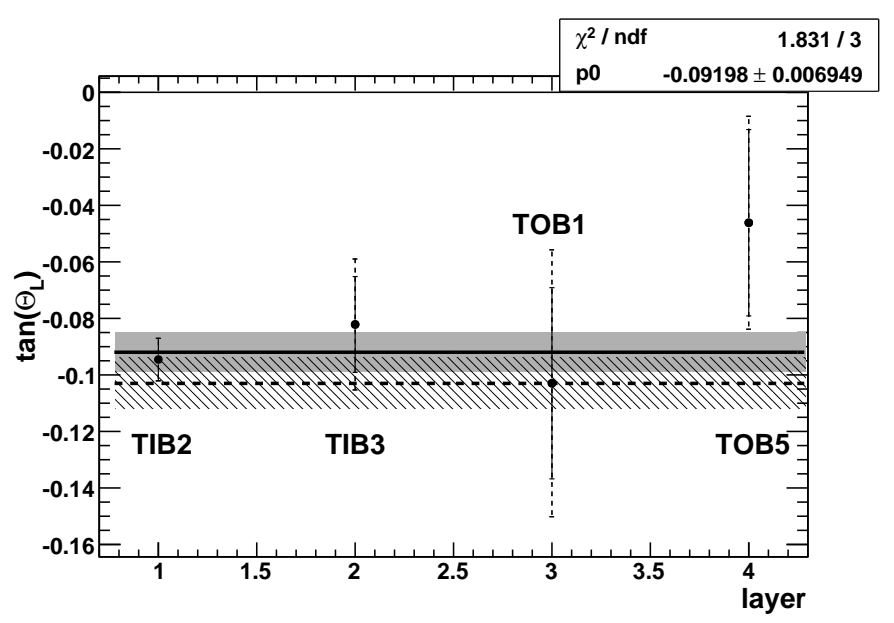

Figure 79. Results of the Lorentz angle measurement in the four different layers using tracks reconstructed by the Road Search algorithm In increasing abscissa value, points correspond to TIB L2 and L3 and TOB L1 and L5. The horizontal line represents the result of the combination of all four layers and the shadow band is the fit uncertainty.The dashed line and the hatched region represent the expected value and his uncertainty, respectively.

Monitoring suite was operated successfully in offline mode, and at the end of the data taking period even in online mode. The synchronization with the Global Trigger was achieved by means of a latency scan, where the signal-to-noise ratio was optimized, reaching the expected values of 28 and 34 respectively for thin and thick silicon sensors. The electronic noise did not change significantly when the magnet was on and the system was powered even during ramping of the current in the coil. Out of the 25 million events collected, over 9000 tracks were reconstructed. Nearly half of these tracks came from data taken with the magnetic field set to $3.8 \mathrm{~T}$ and $4 \mathrm{~T}$. The yield was consistent with the tracker acceptance relative to the Muon System. An alignment procedure was performed, which reduced the hit residuals from $4 \mathrm{~mm}$ to $600 \mu \mathrm{m}$ in the outermost layer. Finally, detector characteristics were studied in depth, including the response function, the optical gain in the front-end electronics, and the Lorentz drift induced by the magnetic field.

The Magnet Test and Cosmic Challenge demonstrated the possibility of operating a subset of the tracker along with the other CMS subsystems and was an important milestone in the installation and commissioning of the final system.

\section{Acknowledgments}

The authors wish to thank all CMS colleagues which made the successful operation of the tracker at SX5 possible: the Run Coordinators, the DAQ group and all colleagues from the other subdetectors. We would also like to express our gratitude to the many technicians who contributed to the assembling and testing of the detector.

\section{References}

[1] C. Jones et al.., The new CMS Event Data Model and Framework, Proceedings of Computing in High-Energy and Nuclear Physics (CHEP06), Mumbai India, 2006. 
[2] The CMS collaboration, CMS: The Tracker Project Technical Design Report, CERN-LHCC-98-006.

[3] The CMS collaboration, The CMS tracker : addendum to the Technical Design Report, CERN-LHCC-2000-016.

[4] M. Frey, The CMS-tracker detector control system, Proceedings of 9th ICATPP Conference on Astroparticle, Particle and Space Physics, Detectors and Medical Physics Applications, Como Italy (2005).

[5] F. Hartmann, The CMS Tracker Control and Safety System, Proceedings of 11th Vienna Conference on Instrumentation, Vienna Austria (2007).

[6] The CMS collaboration, CMS Data Acquisition Technical Design Report, CERN-LHCC-2002-026.

[7] S. Paoletti et al., The Powering Scheme of the CMS Silicon Strip Tracker, Proceedings of 10th Workshop on Electronics for LHC and Future Experiments, Boston U.S.A. (2004).

[8] ETM professional control GmbH, http://www.etm.at.

[9] H. Berger, Automating with STEP7 in STL and SCL, Publicis MCD (2005).

[10] OPC Foundation, http://www.opcfoundation.org.

[11] V. Brigljevic et al., Run Control and Monitor System for the CMS Experiment, Proceedings of Computing in High-Energy and Nuclear Physics (CHEP03), La Jolla U.S.A. (2004), cs.DC/0306110.

[12] PSX package, XDAQ software platform, http://xdaqwiki.cern.ch.

[13] SOAP Protocol, World Wide Web Consortium, http://www.w3.org.

[14] M. Raymond et al., The APV25 $025 \mu \mathrm{m}$ CMOS readout chip for the CMS tracker, Proceedings of 6th Workshop on Electronics for LHC Experiments, Cracow Poland (2000).

[15] J. Troska et al., Optical Readout and Control Systems for the CMS Tracker, IEEE Trans. Nucl. Sci. 50 (2003) 1067 .

[16] J. Coughlan et al., The CMS Tracker Front-End Driver, Proceedings of the 9th Workshop on Electronics for LHC Experiments, Amsterdam The Netherlands (2003), http://cdsweb.cern.ch/record/722058.

[17] F. Drouhin et al., The CERN CMS tracker control system, IEEE Nucl. Sci. Symp. Conf. Rec. 2 (2004) 1196, [CMS-CR-2004-032].

[18] E. Noah et al., Qualification of the CMS Tracker Control Link Digital Optohybrid, Proceedings of the 10th Workshop on Electronics for LHC Experiments, Boston U.S.A. (2004), http://cdsweb.cern.ch/record/814081.

[19] J. Gutleber and L. Orsini, Software Architecture for Processing Clusters Based on $\mathrm{I}_{2} \mathrm{O}$, Cluster Comput. 5 (2002) 55.

[20] H.C. van der Bij et al., S-LINK, a data link interface specification for the LHC era, IEEE Trans. Nucl. Sci. 44 (1997) 398;

S-LINK specifications are available at http://hsi.web.cern.ch/HSI/s-link.

[21] R. Arcidiacono et al., The 2 Tbps "Data to Surface" system of the CMS Data Acquisition, Proceedings of 14th IEEE-NPSS Real Time Conference, Stockholm Sweden (2005), CMS-CR-2006-034.

[22] The CMS collaboration, The TriDAS Project-Technical Design Report, Volume 1: The Trigger Systems, CERN-LHCC-2000-038. 
[23] J. Rehn et al., PhEDEx high-throughput data transfer management system, Proceedings of Computing in High-Energy and Nuclear Physics (CHEP06), Mumbai India (2006).

[24] The CMS collaboration, CMS computing: Technical Design Report, CERN-LHCC-2005-023.

[25] The CMS collaboration, CMS physics : Technical Design Report. Volume I: Detector Performance and Software Physics: Technical Design Report, CERN-LHCC-2006-001.

[26] R. Frühwirth, Application of Kalman filtering to track and vertex fitting, Nucl. Instrum. Meth. A 262 (1987) 444 .

[27] D. Benedetti et al., Tracking and Alignment with the Silicon Strip Tracker at the CMS Magnet Test Cosmic Challenge, CERN-CMS-Note-2007-030.

[28] V. Karimäki et al., The HIP Algorithm for track based alignment and its Application to the CMS pixel detector, CERN-CMS-Note-2006-018.

[29] P. Biallass, T. Hebbeker and K. Hoepfner, Simulation of Cosmic Muons and Comparison with Data from the Cosmic Challenge using Drift Tube Chambers, CERN-CMS-Note-2007-024.

[30] D. Heck et al., CORSIKA: A Monte Carlo code to simulate extensive air showers, Forschungszentrum Karlsruhe FZKA 6019 (1998).

[31] TOSCA is a module of OPERA-3d by Vector Fields Ltd., http://www.vectorfields.com.

[32] S. Dutta et al., Data Quality Monitoring for the CMS Silicon Tracker, Proceedings of Computing in High-Energy and Nuclear Physics (CHEP06), Mumbai India (2006), CMS-CR-2006-012.

[33] G. Alverson et al., The IGUANA Interactive Graphics Toolkit with Examples from CMS and DO, Proceedings of Computing in High-Energy and Nuclear Physics (CHEP01), Beijing China (2001).

[34] M. Axer et al., The Qualification of Silicon Microstrip Detector Modules for the CMS Inner Tracking Detector, CERN-CMS-Note-2006-141.

[35] DEA Hexagon Metrology S.p.A., http://www.dea.it.

[36] S. Dris, K. Gill, J. Troska and F. Vasey, Predicting the Gain Spread of the CMS Tracker Analog Readout Optical Links, CERN-CMS-Note-2006-145.

[37] R. Brauer et al., Design and Test Beam Performance of Substructures of the CMS Tracker End Caps, CERN-CMS-Note-2005-025.

[38] G. Cervelli et al., A linear laser-driver array for optical transmission in the LHC experiments, IEEE Nucl. Sci. Symp. Conf. Rec. 2 (2000) 9/145.

[39] R.A. Smith, Semiconductors, Cambridge University Press, Cambridge U.K. (1968)

[40] V. Ciulli, R. D'Alessandro, S. Frosali and C. Genta, Determination of the Lorentz Angle in Microstrip Silicon Detectors with Cosmic Muons, CERN-CMS-Note-2008-006. 